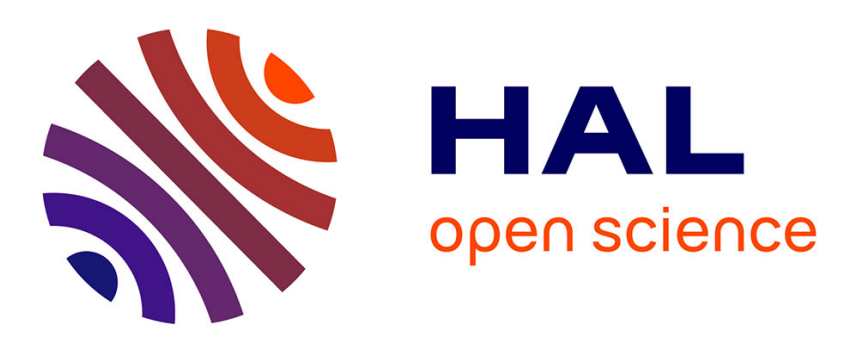

\title{
Least action principle for second gradient continua and capillary fluids: a Lagrangian approach following Piola's point of view
}

Nicolas Auffray, Francesco Dell'Isola, Victor Eremeyev, Angela Madeo, Luca Placidi, Giuseppe Rosi

\section{To cite this version:}

Nicolas Auffray, Francesco Dell'Isola, Victor Eremeyev, Angela Madeo, Luca Placidi, et al.. Least action principle for second gradient continua and capillary fluids: a Lagrangian approach following Piola's point of view. The complete works of Gabrio Piola: Volume I, 38, 2014, Advanced Structured Materials. hal-00955897

\section{HAL Id: hal-00955897 \\ https://hal.science/hal-00955897}

Submitted on 5 Mar 2014

HAL is a multi-disciplinary open access archive for the deposit and dissemination of scientific research documents, whether they are published or not. The documents may come from teaching and research institutions in France or abroad, or from public or private research centers.
L'archive ouverte pluridisciplinaire HAL, est destinée au dépôt et à la diffusion de documents scientifiques de niveau recherche, publiés ou non, émanant des établissements d'enseignement et de recherche français ou étrangers, des laboratoires publics ou privés. 


\title{
Least action principle for second gradient continua and capillary fluids: a Lagrangian approach following Piola's point of view
}

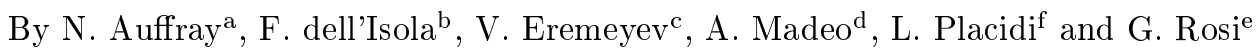

\begin{abstract}
${ }^{a}$ Université Paris-Est, Laboratoire Modélisation et Simulation Multi Echelle, MSME UMR 8208 CNRS, 5 bd Descartes, 77454 Marne-la-Vallée, France
${ }^{\mathrm{b}}$ Dipartimento di Ingegneria Strutturale e Geotecnica, Università di Roma La Sapienza, Via Eudossiana 18, 00184, Roma, Italy

${ }^{\mathrm{c}}$ Institut für Mechanik, Otto-von-Guericke-Universität Magdeburg, 39106 Magdeburg, Germany, and South Scientific Center of RASci \& South Federal University, Rostov on Don, Russia

${ }^{\mathrm{d}}$ Laboratoire de Génie Civil et Ingénierie Environnementale, Université de Lyon-INSA, Bâtiment Coulomb, 69621 Villeurbanne Cedex, France

${ }^{\mathrm{f}}$ International Telematic University Uninettuno, C.so Vittorio Emanuele II, 39, 00186, Rome, Italy

${ }^{\mathrm{e}}$ International Center MeMOCS "Mathematics and Mechanics of Complex System”, Università degli studi dell'Aquila, Palazzo Caetani, Via San Pasquale snc, Cisterna di
\end{abstract} Latina, Italy

“On ne trouvera point de Figures dans cet Ouvrage. Les méthodes que j’y expose ne demandent ni constructions, ni raisonnemens géométriques ou méchaniques, mais seulement des opérations algébriques, assujetties à une marche réguliere et uniforme. Ceux qui aiment l'Analyse, verront avec plaisir la Méchanique en divenir une nouvelle branche, et me sauront gré d'en avoir étendu ansi le domaine."

From theAvertissement of the Méchanique Analitique by Lagrange [87]

\section{Abstract}

As Piola would have surely conjectured, the stationary action principle holds also for capillary fluids, i.e. those fluids for which the deformation energy depends on spatial derivative of mass density (a modelling necessity which has been already remarked by Cahn and Hilliard [15, 16]). For capillary fluids it is indeed possible to define a Lagrangian density function whose corresponding Euler-Lagrange stationarity conditions once transported on the actual configuration, via a Piola's transformation, are exactly those obtained, with different methods, in the literature. We recall that some particulat classes of second gradient fluids are sometimes also called Korteweg-de Vries or Cahn-Allen fluids. More generally those continua (which may be solid or fluid) whose deformation energy depends on the second gradient of placement are called second gradient (or Piola-Toupin or Mindlin or Green-Rivlin or Germain or second grade) continua. In the present work, following closely the procedure first conceived by Piola and carefully presented in his works translated in the present volume, a material (Lagragian) description for second gradient continua is formulated. Subsequently a Lagrangian action is introduced and by means of Piola's transformations this action is calculated in both the material and spatial descriptions. Then the corresponding Euler-Lagrange equations and boundary conditions are calculated by using some kinematical relationships suitably established. Once an objective deformation energy volume density is assumed to depend on either $C$ and $\nabla C$ or on $C^{-1}$ and (where $C$ is the Cauchy-Green deformation tensor) the particular form of aforementioned Euler-Lagrange conditions and boundary conditions are established. When further particularizing the treatment to those energies which characterize fluid materials, the capillary fluid evolution conditions (see e.g. Casal [25] or Seppecher [142, 145] for an alternative deduction based on thermodynamic arguments) are recovered. Also a version of Bernoulli's law which is valid for capillary fluids is found and, in Appendix B, all the kinematic formulas which we have found useful for the present variational formulation are gathered. Many historical comments about Gabrio Piola's contribution to analytical continuum mechanics are also presented when it has been considered useful. In this context the reader is also referred to Capecchi and Ruta [17].

\section{Part I}

\section{Introduction}

Since its first formulation, which can be attributed to D'Alembert and Lagrange at least for what concerns fluid bodies, continuum mechanics has been founded on the principle of virtual work (or principle of virtual velocities, as Lagrange called it). On the other hand, since the early modern ${ }^{1}$ studies on the equilibrium and motion of fluids, the concept of a continuous body has been generally considered

\footnotetext{
${ }^{1}$ It is well known that Archimedes could formulate a precise theory of the equilibrium of fluids (see e.g. Rorres [132]) and there are serious hints that a form of Bernouilli law for fluid flow was known to Hellenistic scientists (see e.g. Vailati [165] or Russo [133]).
} 
adapted to model macroscopic mechanical phenomena even if Poisson [123, 124, 125] claimed, instead, that continuum models had to be based on an atomistic or molecular point of view.

Already Piola [118, 119, 121] presented (most likely for the first time) the rigorous definition (and all related analytical concepts) of the concepts of reference and actual configuration of a continuous system. Therefore he characterized by means of a placement function the physical ideas related to the changes of shape of a deformable body during their motion (those who can understan the Italian language will appreciate the pertinence and elegance of the suggestive expression del movimento di corpi qualsivogliono considerati secondo la naturale loro forma e costituzione used by Gabrio Piola in the title of one of the Memoirs which are translated in this volume). The domain of this function is the original shape of the body while its image is the current shape of the same body.

The reader will recognize that in this modelling need one can trace the origin of many ideas of modern differential geometry. It is clear from the definitions presented by Piola that the space of configurations for a continuous body is an infinite dimensional vector space, or, if necessary, manifold. Therefore one can clearly see and simply state which is the main mathematical difference between discrete and continuous models: the configuration space is finite dimensional in the first case and infinite dimensional in the second one.

Indeed a configuration is characterized as a $n$-tuple of real variables (Lagrange parameters) when introducing discrete models or as a set of suitably defined kinematic fields, defined on suitably fixed domains, when introducing continuous models.

As a consequence the comparison of the two modeling approaches (discrete versus continuous configuration spaces) needs to be developped carefully and has to be based on the different but equally relevant physical aspects of the considered phenomena.

Piola seems to be able to perfom such a comparative analysis and seems to have mastered perfectly the relative mathematical difficulties: the reader is referred to the vivid discussion of this point presented by Piola in his works dated 1845-1846 (see infra in the following subsections and in particular his discussion about reality as perceived by the animaletti infusorj (i.e. micro-organisms)).

On the other hand it seems clear already to Euler, D'Alembert and Lagrange [87] that, in order to formulate an effective model to describe a large class of physical phenomena occurring in deformable bodies, it can be more convenient to introduce a set of space-time partial differential equations for a small number of fields (i.e. functions defined in suitably regular subsets of $\mathbb{R}^{3}$ ) instead of a set of ordinary differential equations in which the set of unknown functions outnumbers any imaginable cardinality.

Since Piola's pioneering work, one of the most fundamental conceptual tools used in continuous models is represented by the definition of the so-called Lagrangian configuration, in which any material particle of the considered continuous body is labeled by three real variables, the material (or Lagrangian) coordinates of the considered particle. As a consequence the motion of a continuous system is characterized by the time dependence of the chosen set of fields.

Even if the structure of their spaces of configurations is different, for both discrete and continuous models the obvious problem arises, once the spaces of configurations are fixed and the set of admissible motions are chosen: how to determine the equations of motion?

In other words:

How one has to model the external interactions between the external world, the considered body and the internal interactions of the body in order to get some evolution equations which, once solved, supply a reliable prediction of the body behavior?

Many different postulation schemes have been developped, during the centuries, have been proposed to solve this problem. For all of these schemes one can find merits and defects: with a somehow inappropriate simplification we may classify them into two subgroups (see a subsection infra) gathered under the collective names analytical continuum mechanics and continuum thermodynamics.

In the opinion of the present authors, Gabrio Piola in his works was perfectly right when he championed the point of view of Lagrange and D'Alembert also in the study of continuum mechanics. His rhetoric elegance seems to be serving a cause which deserves such an emphasis. Indeed the methods of analytical continuum mechanics seem to be the most effective ones (see also [100]), at least when formulating models for mechanical phenomena involving multiple time and length scales.

The reader should consider, with respect to aforementioned class of phenomena, the difficulties which are to be confronted when using continuum thermodynamics, for instance, to describe interfacial phenomena in phase transition (see e.g. dell'Isola and Romano [40, 41, 42] and dell'Isola and Kosinski [43]), or in poroelasticity (see e.g. dell'Isola and Hutter [47]). These difficulties are elegantly overcome when accepting to use as a fundamental tool the principle of virtual work (as done in Casal and Gouin [26], Seppecher [144] and dell'Isola et al. [54]). Related phenomena occur in the flow of bubbles surrounded by their liquid phase: it could be interesting to apply the homogenization techniques presented in Boutin and Auriault [12] to the equations for capillary fluids presented here.

On the other hand tt has to be mentioned that some remarkable results were obtained by combining the two approaches: in the present context one has to cite the works by Seppecher $[142,145]$. In these last papers the author obtained the evolution equations for capillary fluids by combining the principle of virtual work in the Eulerian description with the first principle of thermodynamics (limited to the case of isothermal motions). This shows that it can be sometimes useful to use an heuristic procedure in which the principle of virtual work is reinforced by additionally requiring also the validity of the balance of mechanical energy. Also very interesting in this context are the results presented in Casal [25], Gavrilyuk and Gouin [68]. 


\subsection{Deduction of the evolution equations for capillary fluids and second gradient solids by the principle} of least action

In the present work, following the spirit of the whole scientific production of Gabrio Piola, we show that

it is possible to deduce from the principle of least action, and without any further assumption, the whole set of evolution equations (i.e. bulk equations and boundary conditions) valid for capillary fluids both in the Lagrangian and Eulerian descriptions.

The found evolution equations are the Euler-Lagrange conditions corresponding to a precisely specified action functional.

We expect that the obtained variational principle will be useful at least when formulating numerical schemes for studying a large class of flows of capillary fluids.

Subsequently we observe that a form of Bernoulli's law, valid for capillary fluids, can be proven.

The presented procedure has a structure which allows us to find, without further technical difficultis, with respect to the case of fluid materials, the complete Lagrangian form of the evolution equations for second gradient solids i.e. for materials whose deformation energy is assumed to depend on the deformation measure $C:=F^{T} F$ (where $F$ is the placement gradient with respect to Lagrangian referential coordinates) or, alternatively, on the other equivalent deformation mesure $C^{-1}$ and their gradients in the reference configuration. The obtained equations are valid in the general case of large deformations and large deformation gradients. The appropriate boundary conditions which complete the set of bulk equations are also supplied ${ }^{2}$.

However the spirit of Piola could not be followed in the use of technical tools for obtaining the Euler-Lagrange conditions from postulated Action Functional: indeed the main computational tool that we use is the Levi-Civita tensor calculus, also applied to embedded submanifolds. It has to be remarked that the works of Piola, although correct and rigorous, are encumbered by heavy component-wise notation which hindered their comprehension. Piola's works are truly modern in spirit, except in what concerns their difficulty in treating tensorial quantities: the reader will appreciate the enormous economy of thought which is gained by the use of Levi-Civita formalism.

Piola would not reject the use of more powerful tools for applying Lagrangian and Hamiltonian basic principle as he was indeed aware of the difficulties which are to be confronted when formulating new theories, as is proven by the fact that he claims (see [121], page 1):

"It happens not so seldom that new achievements -by means of which a branch of applied mathematics was augmented-do not appear immediately, in the concept and in the exposition, free from lengthiness and superfluence. The complication of analytical procedures can reach such a level that it could seem impossible to proceed: indeed it is in this moment instead that sometimes a more general point of view can be discovered, many particularities are concentrated, and a compendious theory is formed which is so well grounded that it can infuse vigor for further progresses."

We conclude our introduction by citing a part of the original Introduction of Piola [121], page 5, which is suitable to be included also in the present one $^{3}$, when decontextualizing the references to previous works and replacing the word fluids by capillary fluids:

"While with the present memoir I will aim again to the goal now devised ${ }^{4}$ I will manage to reach also other ones. [Indeed] it is rigorously proved in many places that the general equation of mechanics, written with the notation of the calculus of variations, in the case of a whatsoever discrete system of bodies regarded as points in which different concentrated masses are subjected to external active forces and to internal active and passive forces. However, to start from this last equation [i.e. the equation for a discrete system of points] and to obtain the formulas for the equilibrium and motion of bodies with three dimensional extensions [i.e. deformable bodies], it indeed is a step very difficult for those who are willing to see things clearly and who are not happy with an incomplete understanding. One among my first efforts in this subject can be recognized in my Memoir "On the principles of Analytical mechanics by Lagrange". Published in Milan already in the year 1825, where I presented in this regard some correct ideas but with many specific technical details either too complex or indeed superfluous. I came back to this point in the memoir published in T. XXI of these Atti and I believed to have obtained a remarkable improvement by introducing non-negligible abbreviations and simplifications: but thereafter I perceived the possibility of further improvements which I introduced in the present one. Indeed great advantage can always be obtained when having the care of clarifying appropriately the ideas concerning the nature of different analytical quantities and the spirit of the methods: [to establish] if also from this point of view something has been left to be done, I will leave the judgment to intelligent readers. The scholar will perceive that I propose myself also other aims with the present work, having established here various formulas, which can serve as a starting point for further investigations. I will not omit to mention one of these aims and precisely that one which consists in demonstrating anew (Capo V), by adopting the ideas better founded which are provided by modern Physics about fluids, the fundamental equations of their motions. Insofar as I treated at length in other my works the problems of hydrodynamics (See the first two volumes of Memoirs of I. R. Istituto Lombardo) it was objected that my deduction could be defective, considering what was stated by Poisson about the equations of ordinary Hydrodynamics. Now I believed to be able to prove that the considerations of the French Geometer in this circumstance were pushed too far ahead, and that

\footnotetext{
${ }^{2}$ Some of the found equations are a possible regularization of those proposed e.g. in Yeremeyev et al. [58, 170] for phase transitions in solids and may give an insight into some of the results presented in Eremeyev and Lebedev [61].

${ }^{3}$ The translation from the original italian text tries to reproduce the English style of the famous works by Maxwell [105], which are nearly contemporary with Piola's ones.

${ }^{4}$ Piola refers here to his intention of deducing all the evolution equations of continuum mechanics from the principle of virtual work.
} 
notwithstanding his objections the fundamental theory of the motions of fluids is well grounded as established by D'Alembert and Euler, and exactly as it was reproduced by Fourier himself with the addition of another equation deduced from the theory of heat, [equation] to which, however, it is not necessary to refer in the most obvious questions concerning the science of liquids. For what concerns the motion of fluids, the present Memoir is intended to support and complement the aforementioned ones."

We have gathered in the Appendix B various kinematic formulas, which in our opinion will be useful in further developments of analytical continuum mechanics. The reader should also explicitly note that already Piola has stated that the heat equation (i.e. thermodynamics) does not need to be considered when purely mechanical phenomena are involved.

\subsection{The meanings given to the expressions: second gradient continua and capillary fluids}

As done in Germain [69] we will call second gradient continua those media whose Lagrangian volumetric deformation energy depends both on the first and second gradients of the placement field. When using the expression capillary fluids we will refer to those continua whose Eulerian volumetric deformation energy density depends both on their Eulerian mass density $\rho$ and its gradient $\nabla \rho$. Of course the aforementioned constitutive equations must be independent of the observer (this requirement was already demanded, using the mathematical methods available to him, by Piola [119]). We prefer to avoid naming the introduced class of fluids after Cahn and Hilliard or Korteweg and de Vries, as done sometimes in the literature (See e.g. Seppecher [142, 145, 146, 147, 148], Casal and Gouin [26, 27]). This choice is aimed to try to reduce possible ambiguities: Cahn and Hilliard, for instance, intended the equations which were subsequently named after them to be valid for the concentration of a solute in motion with respect to a stationary solvent, and deduced them via molecular arguments (modulo some thermodynamically relevant terms, see Casal and Gouin [26]). On the other hand the Korteweg-de Vries equations [80] were originally deduced for a completely different class of phenomena: waves on shallow water surfaces. Later it was discovered that they can also be deduced from an atomistic argument, since the so-called Fermi-Pasta-Ulam [63] discrete system has Korteweg-De Vries equations as its continuum limit. Only in a later paper (Korteweg [82]) is a connection with capillarity phenomena established.

The nomenclature capillary fluids has to be preferred because it is suggestive of many of the most fundamental phenomena which may be described by the model discussed here.

Among these (refraining from considering many others) we cite

i) wettability,

ii) the formation of interfacial boundary layers,

iii) the formation of liquid or gaseous films close to walls,

iv) the formation and the motion of drops or bubbles inside another fluid phase or

v) the formation of pendant or sessile drops on a horizontal plane (see e.g. the papers by Seppecher [142, 145], dell'Isola et al. [44], Gouin and Casal [26]).

Finally, we must remark explicitly that second gradient theories are strictly related to continuum theories with microstructure (see e.g. Green, Rivlin [72, 73, 74, 75], Mindlin [106, 107, 108], Kroner [83] and Toupin [160, 161]) as clarified in the note by Bleustein [10] and in the papers by Forest $[65,64]$. Indeed higher gradient theories are simply microstructured continua in which suitable internal constraints are introduced.

\section{An interlude: some apparent dichotomies}

\subsection{Analytical continuum mechanics and continuum thermodynamics}

It is natural and needed to refer at this point to the original sources of analytical continuum mechanics, which indeed seem to be tout court the source of all continuum mechanics. Some of them are relatively close in time and in our opinion, very often, their true spirit has been somehow misjudged or clearly misunderstood.

Sometimes these sources were forgotten or considered by some authors as not being general enough to found modern mechanics.

This is not our opinion: we simply believe that the mathematical formalism needed to construct continuum mechanics starting from variational principles has been regarded as an obstacle to its full understanding and some presentation shortcuts, originally aimed to simplify the mathematical knowledges required to the readers, have indeed hidden the fundamental beauty and elegance of continuum models, whose predictive power cannot be questioned.

One should not believe that simplifying is always useful. Indeed:

i) complexity of physical phenomena cannot be modelled by means of simple models

ii) variational principles supply a powerful tool in creating new models, so that the mathematical abstraction which they involve is repaid by the efficacity of their use in looking for new models for not already described phenomena. 
However, instead of looking for new words to support our point of view, we will continue to cite the champion of analytical mechanics: the Italian mathematical-physicist Gabrio Piola, whose works are translated in this volume. Despite his being one of the founders of modern continuum mechanics, his contribution to it has been seriously underestimated. To our knowledge the appropriate expression analytical continuum mechanics has not been considered frequently up to now. In Maugin [99] the following statement can be found

"The road to the analytical continuum mechanics was explored in particular by P.Germain [71], but not in a variational framework."

The concept underlying analytical continuum mechanics is opposed to those underlying continuum thermodynamics. Actually continuum thermodynamics is based on a postulation process which can be summarized as follows (see e.g. Noll and Truesdell [117], Noll [116]):

- find a set of kinematic fields of relevance in the formulation of the considered continuum model which is sufficient to describe considered phenomena;

- postulate a suitable number of balance laws having the structure of conservation laws. Specify the physical meaning of each conserved quantity and introduce for each a flux, a source and a volume density;

- postulate a suitable number of constitutive equations required to close the formulated mathematical problems: that is to have enough equations to determine the evolution of the kinematic fields, once suitable initial and boundary data are assigned;

- as the possible choices of constitutive equations are too large, and many of them are unphysical, choose a particular balance law, i.e. the balance of entropy, and assume that its source is undeterminate and always positive. The physically acceptable constitutive equations are those for which all possible motions produce a positive entropy.

Anyone who has carefully considered the efficacy of the approach based on continuum thermodynamics may agree that:

- when one wants to formulate new models it is difficult to use it as a heuristic tool;

- it is somehow involved and often requires many ad-hoc assumptions.

We refrain from adding our own comments and concerning the value to be given to the so-called axiomatization of continuum mechanics and to some reasearch programs in the framework of continuum thermodynamics found somewhere in the literature, the interested reader is invited to judge by himself also by referring to two beautiful talks by Rivlin [130,131]. We cite here some exceptions of these two Rivlin's lecture notes with some comments relative to Piola's contribution to mechanics :

"This lecture would not be complete without some reference to the so-called axiomatization of continuum mechanics.

Now, axiomatics carries with it certain overtones of high-living and it becomes just as well to discuss what is the content of this axiomatization of continuum mechanics, particularly as certain impressions which are, in my opinion, quite erroneous have been widely diffused. It has, for example, been presented as fulfilling a program proposed by Hilbert and the impression has been created that it plays much the same role in continuum mechanics as does the axiomatic of Hilbert, say, in pure mathematics. Whatever may be the literal truth of the former claim, it should be understood that the so-called axiomatization of continuum mechanics does not lead to any new unification, paralleling the unification of theorems in widely different branches of mathematics which results from the axiomatization of pure mathematics. Nor has it so far suggested any new and unexpected generalizations, resulting from the abandoning of certain axioms, similar to those which were obtained by abandoning the axiom of parallelism in geometry. Indeed, in content, it appears to be little more than a translation of few familiar concepts in the kinematics and mechanics of continua into the language of elementary set theory" (sect.8 of [131])

It has to be remarked that Gabrio Piola did use very advanced (for his times) mathematical tools: however, in our opinion, with his works he indeed added new physical understanding to mechanical science as his mathematical formalism was really needed to catch relevant phenomena.

"Truesdell's 1952 paper and his subsequent mammoth articles with Toupin and Noll in the Handbuch der Physik [...] have been very valuable in collecting together earlier work and earlier ideas"(sect.12 of [130]).

Indeed Truesdell's review works attracted the attention to Piola's contributions to some parts of continuum mechanics. However the re-discovery of Piola's papers by Truesdell, while attracting the attention of researchers to the now famous Piola's transformation, did obscure the many other novel and original contributions which must be attributed to Piola, as we will try to underline in the present work and as is discussed in the others which are published in this volume.

Again in sect.12 of [130] one can read:

"However, his 1965 article with Noll -The Non-Linear Field Theories of Mechanics- and his voluminous later writings, impressive and admirable though they be in many respects, are seriously marred by his evident contempt for physical reasoning and insight and by a tendency to present the work of his protegï œœs as paradigms, without regard to its originality or its physical or mathematical soundness. In his writings Truesdell evidences a strong taste for dramatic and so there has been created a fantasy world in which various savants 
produce a stream of principles, fundamental theorems, capital results, and works of unusual depth. No matter that, on examination and stripped of the, often irrelevant, mathematical verbiage with which they are surrounded, they frequently turn out to be known results in disguise, or trivial, or physically unacceptable, or mathematically unsound, or some combination of these. Nonetheless, they have been widely and uncritically reproduced in the extensive secondary literature and have provided the starting point for many, correspondingly flawed, theses and papers".

It has to be remarked that, even if he belonged to a much more rhetorical $\ddot{i}_{i} œ p o q u e$ than ours, Piola is very careful in attributing originality and importance to his own results and to those of his contemporaries, even when his nationalistic spirit (see the work about Peridynamics in the same volume) would push him to exalt Italian contributions to mechanical sciences.

A very clear and elegant ${ }^{5}$ comparative analysis of the advantages obtained when physical theories are based on the principle of virtual work (or the principle of least action) is found in classical, although nowadays underestimated article by Hellinger [77]. Actually even a more elegant discussion of this point can be found in Piola (Memoir [121] page 1) where one can read the following words:

"[By means of the concepts of Analytical mechanics] a compendious theory is formed which is so well-grounded that it can infuse vigor for further progresses. It should be desirable that this could happen also for the last additions made by the modern Geometers to Rational mechanics: and in my opinion I should say that the true method suitable to succeed we have in our own hands: it has to be seen if others will be willing to share my opinion. I wrote many times that it does not seem to me needed to create a new mechanics, departing from the luminous method of Lagrange's Analytical mechanics, if one wants to describe the internal phenomena occurring in the motion of bodies: [indeed it is my opinion that] it is possible to adapt those methods to all needs of modern Mathematical Physics : [and that] this is, nay, the true route to follow because, being well grounded in its principles, it leads to reliable consequences and it promises ulterior and grandiose achievements. However I had -and still nowadays I have- as opponents well respected authorities, in front of whom I should concede the point, if the validity of a scientific opinion had to be based on an argument concerning the scientific value of its supporter. Nevertheless, as I cannot renounce to my persuasion, I believed it was suitable to try another effort, gathering in this memoir my thoughts about the subject and having care to expose them with the accuracy needed to assure to them the due attention of Geometers. [...] Even more than for its elegance and the grandiosity of its analytical processes, the true reason for which I prefer to all the other methods in mechanics those methods due to Lagrange is that I see in them the expression of that wise inductive/deductive philosophy brought to us by Newton, which starts from the facts to rise up to the laws and then [starting from established laws] goes down again to the explanation of other facts."

The visionary understanding proven by Piola has been confirmed by the subsequent successes (for instance while phycisists founded Quantun Mechanics) which were obtained in creating new theories based on the principle of least action.

One has to remark, moreover, that analytical continuum mechanics has a much simpler postulation process since, while using it, one has to

- postulate the form of a suitable action functional;

- postulate the form of a suitable dissipation Hamilton-Rayleigh functional, and calculate its first variation with respect to velocity fields;

- assume that in conservative motions the action is stationary, and to determine these motions by calculating the first variation of the action and equating it to zero for every infinitesimal variation of motion;

- equate, for non-conservative motions, the first variation of the action functional (on the infinitesimal variations of motion) to the first variation of the Hamilton-Rayleigh functional with respect to Lagrangian velocities (estimated on the same infinitesimal variations of motion).

The true difficulty in analytical continuum mechanics is that it strongly relies on the methods and on the ideas of the calculus of variations. These methods require to the mechanician who is using them some skills which can be gained only after a suitable mathematical training, which requires a certain time and intelligence investment.

Most likely it is for avoiding all the mathematical abstraction required by the calculus of variations and its ancillary theories that many opponents reject Lagrangian mechanics. This situation repeats cyclically in history of mechanics and was faced by Piola himself.

Again we prefer to give voice to Piola ([121] page 4):

"Somebody could here object that this [i.e. the variational foundations of Analytical mechanics] is a very old knowledge, which does not deserve to be newly promulgated by me: however [it seems that my efforts are needed] as my beautiful theories [after being published] are then criticized, because Poisson has assured us (Mї̈œmoires Institut de France T. VIII. pag. 326, 400; Journal Ecole polyt. cah. XX. pag 2) that the Lagrangian method used for writing the effects of the forces by means of constraint equations (method which is proclaimed here as the only one really suited to take into accounts facts instead of causes) is too abstract; that it is necessary to develop a Science closer

\footnotetext{
${ }^{5}$ This analysis also relatively older when compared with Truesdell's one: but one has to remind that sometimes older does not mean necessarily worse or even
} less advanced! (see [133]) 
to the reality of things; that such analysis [the Lagrangian one] extended to the real bodies must be rejected as insufficient. I respond that I also recognize the difficult question to be in these considerations. If it is well founded or not the statement that the Lagrangian methods are sufficient to the description of all Mechanical Phenomena, and are so powerful that they are suitable for all further possible researches, this is what will be decided later, and before rebutting my point of view, it will be fair to allow me to expose all arguments which I have gathered to defend my point of view. I hope to clarify in the following Memoir that the only reason for which the Analytical Mechanics seemed to be insufficient in the solution of some problems, is that Lagrange, while writing the conditions for equilibrium and motion of a three dimensional body, did not detail his model by assigning the equations relative to every material point belonging to it. If he had done this, and he could very well do it without departing from the methods imparted in his book, he would have obtained easily the same equations at which the French Geometers of our times arrived very painfully, [equations] which now are the foundation of new theories. However those results which Lagrange could not obtain, because death subtracted him from science before he could complete his great oeuvre, indeed those results can be obtained by others."

\subsection{Least (or stationary) action principle and the principle of virtual work}

Very often the principle of least action is considered to be something very different from the principle of virtual work. This is not the case, as was already remarked by Lagrange himself [87]. Actually the principle of least action implies a particular form of the principle of virtual work, and with the addition of a Hamilton-Rayleigh dissipation potential all mechanical models presented in the literature maybe framed into a unique conceptual scheme.

To be more precise we need some specific definitions and notations. Let us consider a physical system denoted $\mathcal{S}$. The set of the possible states this system is mathematically described by a space of configurations $\mathcal{C}$. This space will be endowed with a suitable topological and differential structure, as we will need to define contiuous and differential functions defined in such a space. In particular the time evolution of $\mathcal{S}$ is modeled by a suitably regular function of the time variable whose values belong to $\mathcal{C}$. Following the tradition this function will be called motion function (or shortly: motion).

It is clear that in order to get a well-posed mathematical model for $\mathcal{S}$ one has first establish which is the most suitable space of configurations and then he has to find a set of evolution equations which determine the motions in physically specified conditions.

\section{Least action principle:}

The motions in a time interval $\left[t_{0}, t_{1}\right]$ can be characterized as those motion functions which minimize

(or which are stationary for) a suitably defined action functional in a specified set of admissible motions.

Indeed it is very important, in order to have a well-posed minimization (or stationarity) problem, to precisely specify the set of admissible motions among which these minimizers have to be sought. Following Lagrange it is generally assumed that the set of admissible motions is included in the set of isochronous motions between the instants $t_{0}$ and $t_{1}$, i.e. motions which start from a given configuration at instant $t_{0}$ and arrive to another given configuration at the instant $t_{1}$. When differential calculus is applicable to the action functional, the first variation of this functional (in the sense of Gï̈œteaux derivative) can be estimated. This first variation is a linear continuous functional defined on the set of isochronous infinitesimal variations of motion. In this case, the stationarity condition can be formulated as a differential equation. This equation requires that the first variation to vanish for every infinitesimal variation of motion.

In many of his pioneering works, Lagrange studied a particular class of action functionals (which are now named after him) and gave a method for calculating their first variation under suitable regularity conditions on the action functional itself and on the admissible motions.

The resulting equations of motion are necessary and sufficient conditions (in the class of considered regular motions) for the stationarity of a given action. This method allows for the consideration of both finite- and infinite-dimensional configuration spaces, hence the action principle can be formulated by means of a unique formulation in both cases.

Lagrangian action functionals are given in terms of a suitable Lagrangian function, whose integration in time (and also in space if the configuration space is constituted by spatial fields) is required for calculating the action relative to a given motion. The form of such a function can be regarded as a conjectural choice, whose validity has to be experimentally tested. One can say that a constitutive choice is implicit in the choice of a Lagrange function.

Many opposers of Lagrangian methods object that nobody knows how to choose a Lagrangian for a given physical system. This is an extremely extravagant objection: they indeed complain because a very general (indeed so general that many scientists believe that by means of Lagrangian actions one can model every phenomenon, eventually adding in the picture some dissipation potential) mathematical modelling procedure includes some arbitrary modelling ingredients. These opposers should explain then how they choose the many (and not well-specified) balance equations which they need and how the choose the even more numerous constitutive equations they have to 
postulate: of course no appeal to physical intuition is admissible, as many may consider very intuitive the choice of the Lagragians usually postulated, and the intuition is not a matter of scientific discussion, as is also any question of taste. Indeed the arbitrary choices to be perfomed in the Lagrangian approach are the minimum possible to get a well-posed model and one does not see why modelling procedures which include more arbitrary (and indeed redundant) choices should be preferable.

However, given a configuration space $\mathcal{C}$, one can postulate, instead of a least action principle, a principle of virtual work. Most likely both Piola and the same Lagrange follow D'Alembert in this alternative path in order to avoid some of the most frequent objections which are used against the least action principle.

The principle of virtual work states that the motion of the considered system is uniquely characterized by assuming that for every (admissible) variation the sum of three linear continuous functionals is vanishing.

These functionals represent, respectively,

i) the internal work,

ii) the external work and

iii) the inertial work.

The constitutive choice specifying each of these functionals has a nature similar to the one which leads to a Lagrangian function and is similarly absolutely conjectural in its nature.

As previously stated and as it is required also in the framework of continuum thermodynamics, the validity of these constitutive equations has to be experimentally tested.

It needs to be explicitly remarked that if a Lagrange action functional can be split into three parts, i.e. into the sum of inertial, internal and external terms, the stationarity of action implies the validity of a virtual work principle.

However it is clear that, in general, a linear continuous functional of infinitesimal variations of motion may not be the first variation of a functional necessarily. In this sense then one can state that the principle of virtual works is more general than the principle of least action. The principle of virtual work includes obviously also the principle of least action as modified by Hamilton and Rayleigh.

Therefore, and contrary to what is sometimes stated, both the principle of least action and the principle of virtual work depend on fundamental constitutive assumptions: those which lead to the choice of, respectively, either the three work functionals or the Lagrangian function. The principle of virtual works is, once the configuration space is fixed, able to produce a wider class of motions. In particular it seems to be able to describe a wider class of dissipative phenomena (see e.g. Santilli [134]). However, it has to be remarked that

i) there are dissipative systems which are governed by a least action principle (see e.g. Moiseiwitsch [113] or Vujanovic and Jones [166]);

ii) it is conceivable, by a suitable embedding into a larger space of configuration, to find Lagrangian forms for systems which are initially not Lagrangian (see again Santilli [134] or Carcaterra and Akay [22, 23]).

Whatever may be stated by some savants, the physical insight gained using the principle of least action (or the principle of virtual work) cannot be over estimated.

For a deeper discussion of this point we limit ourselves to cite here, among the vast literature, the textbooks Landau and Lifshitz [85], Lanczos [86], Soper [149], Bedford [8], Kupershmidt [84], Kravchuk and Neittaanmaki [81], Lemons D.S. [90] and the methodological essay by Edwards [56]. Some results of interest in continuum mechanics and structural engineering are gathered in Leipholz [89], and Lippmann [91], while in Luongo and Romeo [93], Luongo et al. [92, 94], are presented some interesting results in the nonlinear dynamics of some structural members.

\subsection{Discrete and continuous models}

In many works (see e.g. Truesdell [162]) it is stated that the principle of least action is suitable to derive the evolution equations for finite

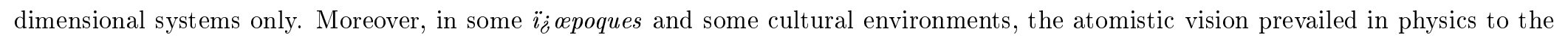
extent that continuum models were considered inappropriate simply for philosophical reasons. Indeed already Poisson bitterly criticized the first works of Piola (see e.g. the introduction of [121]) in which the foundations of modern continuum mechanics are laid based on the principle of virtual work. Actually in Poisson's opinion the true physical reality was atomistic and the most fundamental concept in mechanics was the concept of force, whose balance was bound to lead to the evolution equations of every mechanical system. As a consequence and in order to respond to the objections of Poisson, even if Piola was aware that a variational deduction of the evolution equations for continuous systems was possible, in the first half of the XIX century he decided to regard the continuum theory as the limit of a discrete system. It is interesting to remark that, only a few years later, a similar controversy arose between Mach and Boltzmann, based on Mach's rejection of the atomistic point of view in thermodynamics. We prefer to leave Piola ([121] page 2) to explain his (and our) point of view:

"In my opinion it is not safe enough to found the primordial formulas [of a theory] upon hypotheses which, even being very well-thought, do not receive support if not for a far correspondence with some observed phenomena, correspondence obtained by particularizing general statements, [in my opinion] this should be as coming back in a certain sense to the philosophy of Descartes and Gassendi: indeed the 
magisterium of nature [the experimental evidence] at the very small scale in which we try to conceive the effect of molecular actions will perhaps actually be very different from what we can mentally realize by means of the images impressed in our senses when experiencing their effects on a larger scale. Even let us assume that this difference be very small: a deviation quite insensitive in the fundamental constituents [of matter] -which one needs to consider as multiplied by millions and by billions before one can reach sensible dimensionscan be the ultimate source of notable errors. On the contrary, by using Lagrangian methods, one does not consider in the calculations the actions of internal forces but [only] their effects, which are well-known and are not at all influenced by the incertitude about the effects of prime causes, [so that] no doubt can arise regarding the exactitude of the results. It is true that our imagination may be less satisfied, as [with Lagrangian methods] we do not allow to it to trace the very fundamental origins of the internal motions in bodies: does it really matter? A very large compensation for this deprivation can be found in the certitude of deductions. I could here repeat, if they were not very well-known, the wise documents with which Newton summoned to the science of facts those philosophers who before him had left a too free leap to their imagination. It has to be remarked that I do not intend for this reason to proscribe the dictation of modern Physics about the internal constitution of bodies and the molecular interactions; I think, nay, to render to them the greater of services. When the equations of equilibrium and motion will be established firmly upon indisputable principles, because one has calculated certain effects rather than hypothetical expression of forces, I believe to be licit to try to reconstruct anew these equations by means of [suitable] assumptions about such molecular interactions: and if we manage in this way to get results which are identical to those we already know to be true, I believe that these hypotheses will acquire such a high degree of likeliness which one could never hope to get with other methods. Then the molecular Physics will be encouraged to continue with its deductions, under the condition that, being aware of the aberrations of some bald ancient thinkers, it will always mind to look carefully in the experimental observation those hints /coming by the application of Lagrangian macroscopic methods] which are explicit warnings left there to indicate every eventual deviation."

Regarding the concept of characteristic scale lengths relevant in physical phenomena, Piola had crystal clear ideas, expressed by him with such an elegance that even nowadays his words can be used (Piola [121] page 13):

"Scholium. The admissibility of the principle [i.e. the principle which assumes the existence of a characteristic length $\sigma$ determining the average distance among the molecules microscopically constituting the considered continuum] refers to the true condition of the human being, placed, as said by Pascal in his Thoughts (Part I. Art.IV) at immense distances both from infinity and the zero: distances in which one can imagine many orders of magnitude, of which one [order of magnitude] can be regarded as the whole when compared with the one which is preceding it, and nearly nothing when compared with [the order of magnitude] which follows it. Therefore it results that the same quantities which are asserted to be negligible for us without being afraid of being wrong, could be great and not at all negligible quantities for beings which could be, for instance, capable to perceive the proportions which are relevant for the structure of micro-organisms . For those beings those bodies which appear to us to be continuous could appear as bunches of sacks: water, which for us is a true liquid, could appear as for us [appears] millet or a flowing bunch of lead pellets. But also for these beings there would exist true fluids, relative to which for them the same consequences which we deduce relatively to water should be considered as true. There are therefore quantities which are null absolutely for all orders of beings, as the analytical elements used in the Integral Calculus, and there are quantities which are null only for beings of a certain order, and these quantities would not be null for other beings, as some elements which are considered in mechanics. As I was educated by Brunacci to the school of Lagrange, I always opposed to the metaphysical infinitesimal, as I believe that for the analysis and the geometry (if one wants to achieve clear ideas) it has to be replaced by the indeterminately small when it is needed: however I accept the physical infinitesimal, of which the idea is very clear. It is not an absolute zero, it is nay a magnitude which for other beings could be appreciable, but it is a zero relative to our senses, for which everything which is below them is exactly as if it were not existing.

The reader should remark that the original formulations which lead to the Cahn-Hilliard equations [15, 16] and to capillary fluid equations (see e.g. van Kampen [164], Evans [57], De Gennes [39]) were based on atomistic arguments. However these arguments may lead sometimes to equations (see for more details Casal and Gouin [26]) which are thermodynamically inconsistent. This circumstance was already clear to Piola, who suggested the use of macroscopic theories (based on the principle of virtual work) to derive and confirm the correct deductions from atomistic arguments. This good scientific practice is nowadays generally accepted. Many efforts have been dedicated to deduce from an atomistic scale discrete model the macroscopic form of the deformation energies which depend on first or higher gradients of deformation starting from the works of Piola [121]. The reader is referred to Esposito and Pulvirenti [62] for an extensive review about the results available for fluids. It is suggestive to conjecture that the macro-models for fluid flows discussed e.g. in [7, 28, 78], which involve some micro-macro identification procedure and more than one length scale, may be framed in the general scheme which is put forward here. In solid mechanics also, multiscale models have attracting the interest of many authors: we may refer, for instance, to Sunyk and Steinmann [151], Alibert et al. [1], Steinmann et al. [157], Rinaldi et al. [129], Misra and Chang [109], Yang and Misra [167][168], Yang et al. [169], Misra and Singh [111], Misra and Ching [112] for some other interesting results concerning granular solids. In the same context the results presented in Boutin and Hans [14], Auriault et al. [3], Chesnais et al. [30, 29], Soubestre [150] and Boutin $[13,12]$ have also to be cited. In these papers the authors, although starting in their procedure from balance laws valid at a microscopic level, proceed in a spirit very similar to the one found in the pioneering works by Piola. 


\section{Deduction of evolution equations for continuous systems using the least action principle}

In this part, starting from the least action principle, we present the formal deduction of the evolution equations which govern the motion of i) first gradient continua, in particular Euler fluids, and of ii) second gradient continua, in particular capillary fluids. Although the content of the following subsection is well-known (even if more or less consciously ignored in some literature) it was written pursuing a twofold aim: i) to establish the notation and calculation tools to be used in the subsequent sections; ii) to rephrase there, in a modern notation, the results of Piola $[118,122]$. It has to be remarked that in the literature the least action principle in continuum mechanics is presented in a very clear way in Berdichevsky [9]. It is evident that the Soviet school (see e.g. Sedov [139, 140], which developed, improved and elaborated it in several aspects), was aware of the content of Piola's contribution to continuum mechanics ${ }^{6}$, even if it is not so clear how the information managed to reach Soviet scientists. To establish the ways in which such connections are established is a scientific problem by itself, whose importance has been underestimated up to now.

\section{$3 \quad$ First gradient continua}

In this section we reproduce, by introducing more recent notations and by extensively using Levi-Civita's absolute tensor calculus, the arguments used by Piola for founding the classical continuum mechanics. The reader will observe by simple comparison (see Piola $[118,119,120,121,122])$ that the use of tensor calculus makes the presentation dramatically shorter. Moreover, as we will see in a subsequent subsection, by means of its use the calculations needed to deal with second gradient fluids become feasible. Another difference with Piola's presentation consists in our use of the least action principle instead of the principle of virtual work (see e.g. dell'Isola and Placidi [53]). However we keep the distinction among inertial, internal and external actions. Notations used in the following are detailed in the Appendices.

\subsection{Action functional}

Let us introduce the following action functional:

$$
\mathcal{A}=\Delta_{t_{o}}^{t_{1}} \Delta_{\mathcal{B}}\left(\frac{1}{2} \rho_{0} v^{2}-W(\chi, F, X)\right) d V d t+\Delta_{t_{o}}^{t_{1}} \Delta_{\partial \mathcal{B}}\left(-W_{\mathrm{S}}(\chi, X)\right) d A d t
$$

where :

- the field $\chi$ denotes the placement field between the referential (or Lagrangian) $\mathcal{B}$ and the spatial (or Eulerian) $\chi(\mathcal{B}) \subset \mathcal{E}$ configurations

$$
\chi: \mathcal{B} \rightarrow \mathcal{E}
$$

- the field $\rho_{0}(X)$ refers to the Lagrangian time-independent mass density, so that the Eulerian mass density is given by

$$
\rho=\operatorname{det} F^{-1}\left(\rho_{0}\right)^{\overrightarrow{(\mathcal{E})}}
$$

where the used notation is carefully defined in Appendix A;

- the placement gradient $F=\nabla_{X} \chi$ is a Lagrangian tensor field, i.e. a tensor field defined on $\mathcal{B}$;

- the velocity field $v=\frac{\partial \chi}{\partial t}$, associated to the placement field $\chi$, is a Lagrangian field of Eulerian vectors;

- the potential $W(\chi, F, X)$ is relative to the volumetric density of action inside the volume $\mathcal{B}$;

- the potential $W_{S}(\chi, X)$ pertains to the actions externally applied at the boundary $\partial \mathcal{B}$.

\footnotetext{
${ }^{6}$ It cannot be excluded logically that Piola could have sources which we could not find. However his works fix a date from which certain concepts start to appear
} in published-printed form. 
The results valid for infinite dimensional Lagrangian models (see e.g. dell'Isola and Placidi [53] and references therein) applied to the introduced action, lead to the following Euler-Lagrange equations (which hold at every internal point of $\mathcal{B}$ ):

$$
-\frac{\partial}{\partial t}\left(\rho_{0} v_{i}\right)+\frac{\partial}{\partial X^{A}}\left(\frac{\partial W}{\partial F_{A}^{i}}\right)-\frac{\partial W}{\partial \chi^{i}}=0
$$

and, if the boundary $\partial \mathcal{B}$ is suitably smooth, the following boundary conditions ${ }^{7}$

$$
-\frac{\partial W}{\partial F_{A}^{i}} N_{A}-\frac{\partial W_{\mathrm{S}}}{\partial \chi^{i}}=0
$$

which hold at every point $P$ belonging to the (Lagrangian) surface $\partial \mathcal{B}$ whose normal field is denoted $N(P)$ or, in components, $N_{M}(P)$. In the former expressions and throughout the paper, Lagragian indices are written in upper case while Eulerian indices are written in lower case. Furthermore the classical Einstein convention is applied and the summed indices are taken from the beginning of the alphabet.

\subsection{Objective deformation energy}

We now assume that the energy $W$ can be split into two parts, the first one representing the deformation energy, the second one an external (conservative) action of a bulk load

$$
W(\chi, F, X)=W^{\operatorname{def}}(C, X)+U^{\text {ext }}(\chi, X)
$$

where $C:=F^{T} F$ is the right Cauchy-Green tensor which, in components, has the following expressions:

$$
C_{M N}=g_{N A} F_{a}^{A} F_{M}^{a}=F_{N a} F_{M}^{a}=g_{a b} F_{M}^{b} F_{N}^{a},
$$

where $g_{M N}$ and $g_{i j}$ denotes, respectively, the (distinct) metric tensors over $\mathcal{B}$ and $\mathcal{E}$. The Euler-Lagrange stationarity conditions are the so-called balance of linear momentum, or balance of forces, represented by the equations

$$
-\frac{\partial}{\partial t}\left(\rho_{0} v_{i}\right)+\frac{\partial}{\partial X^{C}}\left(\frac{\partial W^{\text {def }}}{\partial C_{A B}} \frac{\partial C_{A B}}{\partial F_{C}^{i}}\right)-\frac{\partial U^{\text {ext }}}{\partial \chi^{i}}=0
$$

Observe that the equality concerns Eulerian vectors, but the fields are expressed in terms of the Lagrangian variables; therefore the differential operators are Lagrangian. Let us now observe that as:

$$
\frac{\partial C_{M N}}{\partial F_{P}^{i}}=g_{a b} \frac{\partial}{\partial F_{P}^{i}}\left(F_{M}^{b} F_{N}^{a}\right)=g_{a b}\left(\frac{\partial F_{M}^{b}}{\partial F_{P}^{i}} F_{N}^{a}+F_{M}^{b} \frac{\partial F_{N}^{a}}{\partial F_{P}^{i}}\right)=\left(\delta_{M}^{P} F_{i N}+F_{i M} \delta_{N}^{P}\right)
$$

we get

$$
\frac{\partial W^{\text {def }}}{\partial C_{A B}} \frac{\partial C_{A B}}{\partial F_{P}^{i}}=2 \frac{\partial W^{\text {def }}}{\partial C_{P A}} F_{i A}
$$

and the balance (1) becomes

$$
-\rho_{0} \frac{\partial v_{i}}{\partial t}+\frac{\partial}{\partial X^{A}}\left(2 F_{i B} \frac{\partial W^{\text {def }}}{\partial C_{A B}}\right)-\frac{\partial U^{\text {ext }}}{\partial \chi^{i}}=0
$$

The tensor

$$
P_{i}^{M}:=2 F_{i A} \frac{\partial W^{\text {def }}}{\partial C_{A B}} g^{B M}
$$

is the Piola stress tensor. It appears also in the boundary conditions which are deduced from

$$
\frac{\partial W^{\text {def }}}{\partial F_{A}^{i}} N_{A}=-\frac{\partial W_{S}}{\partial \chi^{i}}
$$

In Piola [121] the requirement of objectivity (i.e. the invariance under changes of observer) of Piola stress is clearly stated and analytically formulated. However, due to the lack of conceptual tools supplied by tensor calculus, in his results he did not achieve the clarity allowed by the tensorial formalism.

${ }^{7}$ To avoid any misunderstanding, in the expression $W_{\mathrm{S}}$ the subscript $S$ refers to "Surface" and is not an index. 


\subsection{The Eulerian form of force balance}

Using the Piola transformation (see Appendices), the equations (2), which represent the equations of motion become

$$
-\left(\left.\rho_{0} \frac{\partial v_{i}}{\partial t}\right|_{X}\right)^{\overrightarrow{(\mathcal{E})}}+J^{(\overrightarrow{\mathcal{E}})} \frac{\partial}{\partial x^{a}}\left(2 J^{-1}\left(F_{i A} \frac{\partial W^{\mathrm{def}}}{\partial C_{A B}} F_{B}^{a}\right)^{\overrightarrow{\mathcal{E}})}\right)-\left(\frac{\partial U^{\mathrm{ext}}}{\partial \chi^{i}}\right)^{\overrightarrow{\mathcal{E}})}=0 .
$$

We remark here that $J^{-1}=\operatorname{det}\left(F^{-1}\right)$, consequently $J^{-1}$ has to be considered as an Eulerian quantity. Multiplying this expression by $J^{-1}$ one gets

$$
-J^{-1}\left(\left.\rho_{0} \frac{\partial v_{i}}{\partial t}\right|_{X}\right)^{\overrightarrow{(\mathcal{E})}}+\frac{\partial}{\partial x^{a}}\left(2 J^{-1}\left(F_{i A} \frac{\partial W^{\text {def }}}{\partial C_{A B}} F_{B}^{a}\right)^{\overrightarrow{(\mathcal{E})}}\right)-J^{-1}\left(\frac{\partial U^{\text {ext }}}{\partial \chi^{i}}\right)^{(\overrightarrow{\mathcal{E}})}=0 .
$$

These are recognized as the celebrated balance equations of linear momentum of classical continuum mechanics, once one introduces:

1. the Cauchy stress tensor (which is self-adjoint)

$$
T_{i}^{j}:=2 J^{-1}\left(F_{i A} \frac{\partial W^{\text {def }}}{\partial C_{A B}} F_{B}^{j}\right)^{\overrightarrow{(\mathcal{E})}}
$$

2. the material Eulerian time derivative of a Lagragian field $\Phi$ as

$$
\left(\left.\frac{\partial \Phi}{\partial t}\right|_{X}\right)^{\overrightarrow{(\mathcal{E})}}
$$

3. the field

$$
b^{\text {ext }}:=-J^{-1}\left(\frac{\partial U^{\text {ext }}}{\partial \chi^{i}}\right)^{\overrightarrow{\mathcal{E}})}
$$

which can be called the Eulerian volume force density for considered bulk loads.

Finally, to transport the boundary conditions (3) into the Eulerian configuration, we introduce the following notations, assumptions and results:

1. The body boundary $\partial \mathcal{B}$, whose unit normal field is denoted $N$, is mapped by the placement $\chi$ onto the Eulerian surface $\chi(\partial \mathcal{B})$ whose unit normal field is denoted $n$;

2. Particularizing the relations (43) and (44) provided in the appendix, we obtain that

$$
N_{M}^{\overrightarrow{\mathcal{E}})}=\frac{\left(J^{-1}\left(F^{T}\right)_{M}^{a}\right) n_{a}}{\left\|\left(J^{-1}\left(F^{T}\right)_{A}^{b}\right) n_{b}\right\|}
$$

and that

$$
\frac{d A_{\mathcal{E}}}{d A_{\mathcal{B}}}=\left(\left\|\left(J^{-1}\left(F^{T}\right)_{A}^{a}\right) n_{a}\right\|^{-1}\right)^{(\overrightarrow{\mathcal{B}})}=\left\|\left(J\left(F^{-T}\right)_{a}^{A}\right) N_{A}\right\|
$$

3. The Lagrangian conditions (3) imply

$$
2 \frac{\partial W^{\text {def }}}{\partial C_{A B}} F_{i A} N_{B}=-\frac{\partial W_{S}}{\partial \chi^{i}}
$$

which, by using (6), become

$$
2 F_{i A} \frac{\partial W^{\text {def }}}{\partial C_{A B}} F_{B}^{a}\left(J^{-1} n_{a}\right)^{\overrightarrow{(\mathcal{B})}}=-\frac{\partial W_{S}}{\partial \chi^{i}}\left\|\left(J\left(F^{-T}\right)_{a}^{A}\right) N_{A}\right\|
$$

These last equations, by using (5) and (7), allow us to obtain the well-known Eulerian boundary conditions

$$
T_{i}^{a} n_{a}=\left(-\frac{d A_{\mathcal{E}}}{d A_{\mathcal{B}}} \frac{\partial W_{S}}{\partial \chi^{i}}\right)^{\overrightarrow{\mathcal{E}})}
$$

\subsection{Euler fluids}

We now continue to parallel Piola ([121] Capo V pages 111-146). However our treatment differs since we characterize the material symmetry of Euler fluids by assuming the equation (9) below, while Piola imposes it on the Eulerian transformation of Piola stress. Let us assume 


$$
W^{\text {def }}(C)=\Psi\left(\rho^{\overrightarrow{\mathcal{B}})}(C)\right)=W^{\text {eul }}(F)
$$

and recall the following relations:

$$
\rho^{\overrightarrow{(\mathcal{B})}}=\rho_{0}(\operatorname{det} F)^{-1} ; \quad(\operatorname{det} F)^{2}=\operatorname{det}\left(F^{T} F\right)=\operatorname{det}(C) ; \quad \rho^{\overrightarrow{(\mathcal{B})}}=\rho_{0}(\operatorname{det} C)^{-\frac{1}{2}}
$$

To particularize (4) we need to determine the special form assumed by Cauchy's tensor for Euler fluids. This is done by using:

1. The equality (47) given in the appendices

$$
\frac{\partial \rho^{\overrightarrow{(\mathcal{B})}}}{\partial C_{M N}}=-\frac{\rho^{\overrightarrow{(\mathcal{B})}}}{2}\left(F^{-1}\right)^{M a}\left(F^{-1}\right)_{a}^{N}
$$

2. The equality

$$
T_{i}^{j}=2 J^{-1}\left(F_{i A} \frac{\partial \Psi}{\partial \rho^{\overrightarrow{(\mathcal{B})}}} \frac{\partial \rho^{\overrightarrow{(\mathcal{B})}}}{\partial C_{A B}} F_{B}^{j}\right)^{\overrightarrow{(\mathcal{E})}}=-J^{-1} \rho \frac{\partial \Psi}{\partial \rho} \delta_{i}^{a} \delta_{a}^{j}=-\rho^{2} \frac{\partial\left(\Psi / \rho_{0}^{(\overrightarrow{\mathcal{E}})}\right)}{\partial \rho} \delta_{i}^{j}
$$

3. The definition of the constitutive equation giving the pressure as a function of density

$$
p(\rho):=\rho^{2} \frac{\partial\left(\Psi / \rho_{0}^{\overrightarrow{\mathcal{E}})}\right)}{\partial \rho}
$$

In conclusion, by using (11) and (10), the Eulerian force balance equations assume the form:

$$
-\rho_{0}^{\overrightarrow{\mathcal{E}})} J^{-1}\left(\left.\frac{\partial v_{i}}{\partial t}\right|_{X}\right)^{\overrightarrow{\mathcal{E}})}-\frac{\partial p(\rho)}{\partial x^{i}}-J^{-1}\left(\frac{\partial U}{\partial \chi^{i}}\right)^{\overrightarrow{\mathcal{E}})}=0
$$

By considering the external potential energy per unit mass, the last equation reads

$$
-\rho\left(\left.\frac{\partial v_{i}}{\partial t}\right|_{X}\right)^{\overrightarrow{(\mathcal{E})}}-\frac{\partial p(\rho)}{\partial x^{i}}-\rho\left(\frac{\partial\left(U / \rho_{0}\right)}{\partial \chi^{i}}\right)^{\overrightarrow{(\mathcal{E})}}=0 .
$$

Finally, using the formula for calculating the material derivative of velocity we obtain

$$
-\rho\left(\frac{\partial v_{i}^{\overrightarrow{(\mathcal{E})}}}{\partial t}+\frac{\partial v_{i}^{\overrightarrow{(\mathcal{E})}}}{\partial x^{a}}\left(v^{a}\right)^{\overrightarrow{(\mathcal{E})}}\right)-\frac{\partial p(\rho)}{\partial x^{i}}-\rho\left(\frac{\partial\left(U / \rho_{0}\right)}{\partial \chi^{i}}\right)^{\overrightarrow{(\mathcal{E})}}=0
$$

The expression (10) for the Cauchy stress, which is valid for Euler fluids, together with the boundary condition (8) implies that:

Not all externally applied actions can be sustained by Euler fluids. Indeed Euler fluids cannot sustain arbitrary surface tractions (as pressure is always positive) nor surface shear forces.

This statement, which can be already found in Piola [121] (see equation (37) page 136 and the subsequent discussion), implies that:

"The assumptions about the internal deformation energy determine the capability of the considered body to sustain externally applied actions. Therefore: the expression of the internal deformation energy characterizes the class of admissible external actions of a continuous body." We will return on this point in the next sections.

\section{Second gradient continua}

In this section we generalize the expression for deformation energy used up to now to take the second gradient of the displacement field into account. It has to be remarked that in Piola ([121] page 152) a first (and persuasive!) argument supporting the possible importance of dependence of the internal work functional on higher gradients of displacement field is put forward. This point deserves a deeper discussion and is postponed to future investigations. To our knowledge Piola is the first author who analyzed such a dependence. Therefore we 
propose to name after him the obtained generalized continuum theories. It is assumed that second gradient materials have a deformation energy which depends both on the Cauchy-Green tensor and on its first gradient. The more general Lagrangian density function to be considered has the following form

$$
\mathcal{L}=\frac{1}{2} \rho_{0} v^{2}-\left(W^{I}(\chi, F, X)+W^{I I}(\chi, F, \nabla F, X)\right) .
$$

\subsection{Piola-type second gradient deformation energy}

The expression (12) will be assumed in the sequel. The term $W^{I}(\chi, F, X)$ coincides with the first order term previously considered, while $W^{I I}(\chi, F, \nabla F, X)$ stands for an additive term in which the first order derivative of the gradient $F$ appears. As a consequence, we need to compute the first variation of the following functional

$$
\mathcal{A}^{I I}=\Delta_{\mathcal{B}}-W^{I I}(\chi, F, \nabla F, X) d V
$$

Paralleling the style of presentation used by Piola, while developing the calculations we comment on the results as soon as they are obtained. Because of the assumed structure of the added deformation energy, we have

$$
\begin{aligned}
\delta \mathcal{A}^{I I} & =\delta_{\chi} \mathcal{A}^{I I}+\delta_{F} \mathcal{A}^{I I}+\delta_{\nabla F} \mathcal{A}^{I I} \\
& =\Delta_{\mathcal{B}}-\left(\frac{\partial W^{I I}(\chi, F, \nabla F, X)}{\partial \chi} \delta \chi+\frac{\partial W^{I I}(\chi, F, \nabla F, X)}{\partial F} \delta F+\frac{\partial W^{I I}(\chi, F, \nabla F, X)}{\partial \nabla F} \delta \nabla F\right) d V
\end{aligned}
$$

It can be observed that the first two terms can be treated exactly in the manner of the first gradient action. The following expression in the bulk equation will be obtained

$$
D I V_{X}\left(\frac{\partial W^{I I}}{\partial F}\right)-\frac{\partial W^{I I}}{\partial \chi}
$$

together with the following term in the boundary conditions:

$$
-\frac{\partial W^{I I}}{\partial F} \cdot N
$$

On the contrary, new difficulties appear when calculating the first variation $\delta_{\nabla F} \mathcal{A}$. However, the techniques developed by Mindlin, Green, Rivlin, Toupin and Germain (see also dell'Isola et al. [54]) allow us to treat this term efficiently and elegantly. Starting from (the comma indicates partial differentiation $)^{8}$

$$
\delta_{\nabla F} \mathcal{A}^{I I}=\Delta_{\mathcal{B}}-\left(\frac{\partial W^{I I}}{\partial F_{A, B}^{a}} \delta F_{A, B}^{a}\right) d V
$$

we perform a first integration by parts. Indeed remarking that

$$
\frac{\partial}{\partial X^{B}}\left(\frac{\partial W^{I I}}{\partial F_{A, B}^{a}} \delta F_{A}^{a}\right)=\frac{\partial}{\partial X^{B}}\left(\frac{\partial W^{I I}}{\partial F_{A, B}^{a}}\right) \delta F_{A}^{a}+\frac{\partial W^{I I}}{\partial F_{A, B}^{a}} \delta F_{A, B}^{a}
$$

and applying the divergence theorem (recall that we denote by $N_{M}$ the components of the unit normal to the surface $\partial \mathcal{B}$ ), we obtain

$$
\begin{aligned}
\delta_{\nabla F} \mathcal{A}^{I I} & =\Delta_{\mathcal{B}}-\left(\frac{\partial W^{I I}}{\partial F_{A, B}^{a}} \delta F_{A, B}^{a}\right) d V=-\Delta_{\mathcal{B}} \frac{\partial}{\partial X^{B}}\left(\frac{\partial W^{I I}}{\partial F_{A, B}^{a}} \delta F_{A}^{a}\right) d V+\Delta_{\mathcal{B}}\left(\frac{\partial}{\partial X^{B}}\left(\frac{\partial W^{I I}}{\partial F_{A, B}^{a}}\right) \delta F_{A}^{a}\right) d V \\
& =-\Delta_{\partial \mathcal{B}}\left(\frac{\partial W^{I I}}{\partial F_{A, B}^{a}} N_{B}\right) \delta F_{A}^{a} d A+\Delta_{\mathcal{B}}\left(\frac{\partial}{\partial X^{B}}\left(\frac{\partial W^{I I}}{\partial F_{A, B}^{a}}\right) \delta F_{A}^{a}\right) d V .
\end{aligned}
$$

Let us observe that the second term of the previous expression has exactly the same form as the first variation of the first gradient action.

Therefore this term becomes

$$
\begin{aligned}
\Delta_{\mathcal{B}}\left(\frac{\partial}{\partial X^{B}}\left(\frac{\partial W^{I I}}{\partial F_{A, B}^{a}}\right) \delta F_{A}^{a}\right) d V & =\Delta_{\mathcal{B}} \frac{\partial}{\partial X^{A}}\left(\frac{\partial}{\partial X^{B}}\left(\frac{\partial W^{I I}}{\partial F_{A, B}^{a}}\right) \delta \chi^{a}\right) d V-\Delta_{\mathcal{B}}\left(\frac{\partial^{2}}{\partial X^{A} \partial X^{B}}\left(\frac{\partial W^{I I}}{\partial F_{A, B}^{a}}\right) \delta \chi^{a}\right) d V \\
& =\Delta_{\partial \mathcal{B}}\left(N_{A} \frac{\partial}{\partial X^{B}}\left(\frac{\partial W^{I I}}{\partial F_{A, B}^{a}}\right)\right) \delta \chi^{a} d A-\Delta_{\mathcal{B}}\left(\frac{\partial^{2}}{\partial X^{A} \partial X^{B}}\left(\frac{\partial W^{I I}}{\partial F_{A, B}^{a}}\right) \delta \chi^{a}\right) d V
\end{aligned}
$$

\footnotetext{
${ }^{8}$ In this paper we introduce in both referential and spatial configurations a chart with vanishing Christoffel symbol so that covariant derivatives coincide with
} derivatives. 
which implies that to (13) and (14) the following terms must, respectively, be added to the bulk and surface Euler-Lagrange conditions

$$
-D I V_{X}\left(D I V_{X}\left(\frac{\partial W^{I I}}{\partial \nabla F}\right)\right) ; \quad D I V_{X}\left(\frac{\partial W^{I I}}{\partial \nabla F}\right) \cdot N
$$

\section{2 $\quad$ First gradient surface stress}

We now have to treat the first term in (15), performing a surface integration by parts. We obtain

$$
-\Delta_{\partial \mathcal{B}}\left(\frac{\partial W^{I I}}{\partial F_{A, B}^{a}} N_{B}\right) \delta F_{A}^{a} d A
$$

Recall that in dell'Isola et al. [54] the factor

$$
\left(\frac{\partial W^{I I}}{\partial F_{A, B}^{a}} N_{B}\right)
$$

appearing in a virtual work functional of the kind given in (16) was called first gradient surface stress. To proceed in the calculations we need to use some results from Gaussian differential geometry (see e.g. Appendices for more details). The main tool we use consists in the introduction of two projector fields $P$ and $Q$ in the neighborhood of the surface $\partial \mathcal{B}$. The operator $P$ projects onto its tangent plane, while $Q$ projects on the normal. The used integration-by-parts techniques were introduced to us by Seppecher [141]. They are developed in the framework of Levi-Civita absolute tensor calculus, however it is clear that the sources of Berdichevsky [9] systematically employed these techniques. With their help, the expression (16) is transformed in the following way

$$
\begin{aligned}
-\Delta_{\partial \mathcal{B}}\left(\frac{\partial W^{I I}}{\partial F_{A, B}^{a}} N_{B}\right) \delta \chi_{, A}^{a} d A & =-\Delta_{\partial \mathcal{B}}\left(\frac{\partial W^{I I}}{\partial F_{A, B}^{a}} N_{B}\right) \delta \chi_{, C}^{a} \delta_{A}^{C} d A=-\Delta \partial \mathcal{B}\left(\frac{\partial W^{I I}}{\partial F_{A, B}^{a}} N_{B}\right) \delta \chi_{, C}^{a}\left(Q_{A}^{C}+P_{A}^{C}\right) d A \\
& =-\Delta \partial \mathcal{B}\left(\frac{\partial W^{I I}}{\partial F_{A, B}^{a}} N_{B}\right) \delta \chi_{, C}^{a} Q_{D}^{C} Q_{A}^{D} d A-\Delta \partial \mathcal{B}\left(\frac{\partial W^{I I}}{\partial F_{A, B}^{a}} N_{B}\right) \delta \chi_{, C}^{a} P_{D}^{C} P_{A}^{D} d A
\end{aligned}
$$

In the following subsections, each elementary term will be discussed.

\subsection{External and contact surface double forces}

Considering that

$$
Q_{D}^{C}:=N^{C} N_{D}
$$

the first term in equation (17) is rewritten

$$
-\Delta_{\partial \mathcal{B}}\left(\frac{\partial W^{I I}}{\partial F_{A, B}^{a}} N_{B}\right) \delta \chi_{, C}^{a} Q_{D}^{C} Q_{A}^{D} d A=-\Delta \partial \mathcal{B}\left(\frac{\partial W^{I I}}{\partial F_{A, B}^{a}} N_{B}\right) \delta \chi_{, C}^{a} N^{C} N_{D} N^{D} N_{A} d A=-\Delta \partial \mathcal{B}\left(\frac{\partial W^{I I}}{\partial F_{A, B}^{a}} N_{B} N_{A}\right)\left(\delta \chi_{, C}^{a} N^{C}\right) d A
$$

or, in coordinate-free form,

$$
-\Delta_{\partial \mathcal{B}}\left(\frac{\partial W^{I I}}{\partial \nabla F} \cdot(N \otimes N)\right) \cdot\left(\frac{\partial \delta \chi}{\partial N}\right) d A .
$$

This last expression cannot be reduced further, and makes clear the appearance of a new kind of boundary condition. This quantity represents the work expended on the independent kinematical quantity

$$
\frac{\partial \delta \chi}{\partial N}
$$

by its dual action, which is sometimes called a double force (see e.g Germain [69]), namely

$$
\frac{\partial W^{I I}}{\partial \nabla F} \cdot(N \otimes N)
$$

Actually the appearance of the work functional (18) justifies the following statement, which fits in the spirit of Piola [121] and is reaffirmed in Berdichevsky [9]:

Second gradient continua can sustain external surface double forces, i.e. external actions expending work on the virtual normal gradient of displacement fields. 
As a consequence, in the action functional, one is allowed to add a term of the kind:

$$
\mathcal{A}_{S}^{I I}=\Delta_{t_{o}}^{t_{1}} \Delta \partial \mathcal{B}\left(-W_{S}^{I I}\left(\chi, \frac{\partial \chi}{\partial N}, X\right)\right) d A d t
$$

where the potential $W_{S}^{I I}\left(\chi, \frac{\partial \chi}{\partial N}, X\right)$ can be called surface external double potential.

\subsection{Edge contact forces}

The term expressing the work expended on virtual displacement fields parallel to the tangent space to $\partial \mathcal{B}$, namely

$$
\Delta_{\partial \mathcal{B}} \delta \chi_{, C}^{a} P_{D}^{C} P_{A}^{D}\left(\frac{\partial W^{I I}}{\partial F_{A, B}^{a}} N_{B}\right) d A
$$

can be reduced by means of an integration by parts in the submanifold $\partial \mathcal{B}$ to

$$
\Delta_{\partial \mathcal{B}}\left(\delta \chi_{, C}^{a} P_{A}^{D} \frac{\partial W^{I I}}{\partial F_{A, B}^{a}} N_{B}\right) P_{D}^{C} d A=\Delta \partial \mathcal{B} \frac{\partial}{\partial X^{C}}\left(P_{A}^{D} \frac{\partial W^{I I}}{\partial F_{A, B}^{a}} N_{B} \delta \chi^{a}\right) P_{D}^{C} d A-\Delta_{\partial \mathcal{B}} \frac{\partial}{\partial X^{C}}\left(P_{A}^{D} \frac{\partial W^{I I}}{\partial F_{A, B}^{a}} N_{B}\right) \delta \chi^{a} P_{D}^{C} d A
$$

Surface divergence theorem is then applied to the first term, resulting in the following equality (see Appendices or dell'Isola et al. [54])

$$
\Delta_{\partial \mathcal{B}} \frac{\partial}{\partial X^{C}}\left(P_{A}^{D} \frac{\partial W^{I I}}{\partial F_{A, B}^{a}} N_{B} \delta \chi^{a}\right) P_{D}^{C} d A=\Delta \partial \partial \mathcal{B}\left(\frac{\partial W^{I I}}{\partial F_{A, B}^{a}} N_{B} \delta \chi^{a}\right) P_{A}^{C} \nu_{C} d L=\Delta \partial \partial \mathcal{B} \delta \chi^{a}\left(\frac{\partial W^{I I}}{\partial F_{A, B}^{a}} N_{B} \nu_{A}\right) d L
$$

When the surface $\partial \mathcal{B}$ is orientable and $C^{1}$, the boundary $\partial \partial \mathcal{B}$ is empty. Alternatively, if $\partial \mathcal{B}$ is piecewise $C^{1}$ then $\partial \partial \mathcal{B}$ is the union of the edges of $\partial \mathcal{B}$ and the obtained expression represents the work expended by contact edge forces on the virtual displacement $\delta \chi$. To the boundary conditions it is therefore necessary to add on $\partial \partial \mathcal{B}$ the following terms, which balance external line forces

$$
\frac{\partial W^{I I}}{\partial F_{A, B}^{i}} N_{B} \nu_{A}
$$

Once again, the appearance of the work functional (20) justifies the following statement:

Second gradient continua can sustain external line forces, i.e. external actions expending work on virtual displacement fields at the edges of the boundary $\partial \mathcal{B}$.

This means that, in the action functional, one is allowed to add a term of the kind:

$$
\mathcal{A}_{L}^{I I}=\Delta_{t_{o}}^{t_{1}} \Delta_{\partial \partial \mathcal{B}}\left(-W_{L}^{I I}(\chi, X)\right) d L d t
$$

where the potential $W_{L}^{I I}(\chi, X)$ can be called line external potential.

\subsection{Contact forces depending on curvature of contact surfaces}

The second term of equation (19) produces a further term to be added to surface boundary conditions, which can be interpreted as a new kind of contact force (as it expends work on virtual displacements). The newly (by Casal, Mindlin, Green, Rivlin and Germain) found contact force does not obey the so-called Cauchy postulate, as it depends not only on the normal of Cauchy cuts but also on their curvature. The surface boundary conditions have to be complemented by the following terms

$$
-D I V_{\partial \mathcal{B}}\left(P\left(\frac{\partial W^{I I}}{\partial \nabla F} \cdot N\right)\right)
$$

which depend explicitly on the curvature of the surface $\partial \mathcal{B}$.

\subsection{Resumï ¿œ of terms to be added to Euler-Lagrange equations for second gradient continua}

The Euler-Lagrange conditions found for first gradient action have to be completed by the terms listed below (see [45, 46]): 
- terms to be added to bulk equations

$$
D I V_{X}\left(\frac{\partial W^{I I}}{\partial F}\right)-\frac{\partial W^{I I}}{\partial \chi^{i}}-D I V_{X}\left(D I V_{X}\left(\frac{\partial W^{I I}}{\partial \nabla F}\right)\right)
$$

- terms to be added to surface boundary conditions

$$
-\frac{\partial W^{I I}}{\partial F} \cdot N+D I V_{X}\left(\frac{\partial W^{I I}}{\partial \nabla F}\right) \cdot N-D I V_{\partial \mathcal{B}}\left(P\left(\frac{\partial W^{I I}}{\partial \nabla F} \cdot N\right)\right)
$$

- terms to be added to form new edge boundary conditions

$$
\frac{\partial W^{I I}}{\partial \nabla F} \cdot(N \otimes \nu)-\frac{\partial W_{L}^{I I}(\chi, X)}{\partial \chi}
$$

- terms forming new surface boundary conditions (which may be called balance of contact double forces)

$$
\frac{\partial W^{I I}}{\partial \nabla F} \cdot(N \otimes N)-\frac{\partial W_{S}^{I I}\left(\chi, \frac{\partial \chi}{\partial N}, X\right)}{\partial\left(\frac{\partial \chi}{\partial N}\right)}
$$

\subsection{Objective second gradient energies}

The added term

$$
W^{I I}(\chi, F, \nabla F, X)
$$

must of course be invariant under the change of the observer in the Eulerian configuration. The use of the Cauchy-Green deformation tensor ensures that the deformation energy is objective (see e.g. [52]). This requirement is verified by a deformation energy having one of the forms

$$
\hat{W}^{I I}(C, \nabla C, X) ; \quad \check{W}^{I I}\left(C^{-1}, \nabla C^{-1}, X\right)
$$

It is interesting to remark that many continuum models of fiber reinforced materials (see e.g Steigmann [152], Atai and Steigmann [2], Nadler and Steigmann [114], Nadler et al. [115], Haseganu and Steigmann [76]) show some peculiarities which can be explained by the introduction of second gradient or even higher gradient models. Therefore, in order to calculate the partial derivatives with respect to $F$ and $\nabla F$ appearing in the equations $(21),(22),(23)$ and $(24)$, it is necessary to calculate the derivatives listed in the following formulas (see Appendix 8 for more details ).

- Derivatives of $C$ and $\nabla C$ :

$$
\begin{gathered}
\frac{\partial C_{M N}}{\partial F_{P}^{i}}=\delta_{M}^{P} F_{i N}+F_{i M} \delta_{N}^{P} \\
\frac{\partial C_{M N, O}}{\partial F_{P}^{i}}=F_{i M, O} \delta_{P}^{N}+F_{i N, O} \delta_{P}^{M} \\
\frac{\partial C_{M N, O}}{\partial F_{P, Q}^{i}}=\delta_{M}^{P} \delta_{O}^{Q} F_{N i}+\delta_{N}^{P} \delta_{O}^{Q} F_{M i}
\end{gathered}
$$

- Derivatives of $C^{-1}$ and $\nabla C^{-1}$ :

$$
\begin{gathered}
\frac{\partial C_{M N}^{-1}}{\partial F_{P}^{i}}=-\left(F^{-1}\right)_{M i}\left(F^{-1}\right)_{a}^{P}\left(F^{-1}\right)_{N}^{a}-\left(F^{-1}\right)_{N i}\left(F^{-1}\right)^{b P}\left(F^{-1}\right)_{b M} \\
\frac{\partial C_{M N, O}^{-1}}{\partial F_{P}^{l}}=-\left(F^{-1}\right)^{a P}\left(\left(F^{-1}\right)_{N l}\left(F^{-1}\right)_{M a, O}+\left(F^{-1}\right)_{M l}\left(F^{-1}\right)_{a N, O}\right) \\
\frac{\partial C_{M N, O}^{-1}}{\partial F_{P, Q}^{i}}=-\left[\left(F^{-1}\right)_{M i}\left(F^{-1}\right)^{a P}\left(F^{-1}\right)_{a N}+\left(F^{-1}\right)_{N i}\left(F^{-1}\right)^{b P}\left(F^{-1}\right)_{b M}\right] \delta_{Q}^{O}
\end{gathered}
$$

\subsection{Capillary fluids}

In Poisson [125] pages 5-6: (translated by the authors) one finds the following statements about the region of a fluid in which a phase transition occurs (page 5)

"But Laplace omitted, in his calculations, a physical circumstance whose consideration is essential: I refer to the rapid variation of density which the liquid experiences in proximity of its free surface and of the tube wall, [variation] without which the capillary phenomena 
could not occur [...] Actually, in an equilibrium state, each layer infinitely thin of a liquid is compressed equally on both of its faces by the repulsive actions of all close molecules diminished by their attractive force [....] and its level of condensation is determined by the magnitude of the compressive force. At a sensible distance from the surface of the liquid the aforementioned force is exerted by a liquid layer adjacent to the infinitely thin layer, whose thickness is complete and everywhere constant, i.e. equal to the radius of activity of fluid molecules; and for this reason the internal density of the liquid is also constant [...] But when this distance is less than the radius of molecular activity the thickness of the layer under the layer which we are considering is also smaller than this radius: the compressive force which is exerted by the said upper layer is therefore decreasing very rapidly with the distance from the surface and vanishes at the surface itself, where the infinitesimal thin layer is compressed only by the atmospheric pressure. Consequently, the condensation of the liquid is also decreasing, following an unknown law, when one is approaching its free surface and its density is very different in that surface and at a depth which exceeds by a small amount the activity radius of its molecules, which is sufficient for having this density to be equal to the internal density of the liquid. Now it will be proven in the first chapter of this work that if one neglects this rapid variation of density in the thickness of the interfacial layer ${ }^{9}$ then the capillary surface should result to be plane and horizontal and one could not observe neither elevation nor lowering of the liquid level.[...]"

Therefore we can conclude that already Poisson wanted, with some assumptions which probably need to be clarified, to model the interfacial layer as a thin but three-dimensional layer. It is interesting to remark that it is only because of the development of the ideas by Piola (ideas which Poisson violently criticized) that the modern theory of capillary fluids managed to give a precise meaning to the Poisson's intuitions. What Poisson calls an unknown law is now explicitly determined by using second gradient continua (see e.g. [24, 142]).

In the spirit of Piola's works, we now consider the most simple class of second gradient continua, i.e. capillary fluids. We recall here that capillary fluids are continua whose Eulerian volumetric deformation energy density depends both on their Eulerian mass density $\rho$ and on its gradient $\nabla \rho$. For capillary fluids an additive extra term in the part of action related to deformation energy has to be considered:

$$
\mathcal{A}^{\text {cap }}=\Delta_{\mathcal{E}} \hat{W}^{\text {cap }}(\rho, \nabla \rho) d v=\Delta_{\mathcal{B}} J \hat{W}^{\text {cap }}\left((\rho)^{(\overrightarrow{\mathcal{B}})},(\nabla \rho)^{(\overrightarrow{\mathcal{B}})}\right) d V
$$

The notations $(\cdot)^{\overrightarrow{\mathcal{B}})}$ and $(\cdot)^{\overrightarrow{(\mathcal{E})}}$ introduced in the Appendix A, will be omitted occasionally for the sake of readability. Obviously the dependence of $\hat{W}^{\text {cap }}$ on $\nabla \rho$ must be objective. Therefore we must have (see e.g. Ball [5])

$$
\hat{W}^{\text {cap }}(\rho, \nabla \rho)=\check{W}^{\text {cap }}(\rho, \beta)
$$

where we introduced the scalar

$$
\beta:=\nabla \rho \cdot \nabla \rho .
$$

A particular case of the energy (25) is given by the one discussed by Cahn and Hilliard

$$
\check{W}^{\text {cap }}(\rho, \beta)=\frac{1}{2} \lambda(\rho) \beta=\frac{1}{2} \lambda(\rho)(\nabla \rho \cdot \nabla \rho)
$$

where the function $\lambda(\rho)$ has been often considered to be constant.

\subsubsection{Lagrangian expression for the deformation energy of capillary fluids}

It is therefore needed to calculate the following first variation

$$
\delta \mathcal{A}^{\text {cap }}=\delta\left(\Delta_{\mathcal{B}} J \check{W}^{\text {cap }}\left((\rho)^{\overrightarrow{(\mathcal{B})}},(\beta)^{(\overrightarrow{\mathcal{B}})}\right) d V\right) .
$$

Once we have defined (with an abuse of notation)

$$
W^{\text {cap }}(F, \nabla F):=J \check{W}^{\text {cap }}\left((\rho)^{\overrightarrow{(\mathcal{B})}},(\beta)^{\overrightarrow{(\mathcal{B})}}\right)=\frac{\rho_{0}}{(\rho)^{(\overrightarrow{\mathcal{B}})}} \check{W}^{\text {cap }}
$$

\footnotetext{
${ }^{9}$ This thickness must have a finite value but this value must be undetectable not sensible, because of the hypothesis which was accepted about the extension of
} molecular activity. This is confirmed by the experience made by M.Gay-Lussac. 


$$
\begin{aligned}
\delta\left(J \check{W}^{\text {cap }}\right) & =\left(\check{W}^{\text {cap }} \delta J+J \frac{\partial \check{W}^{\text {cap }}}{\partial(\rho)^{\overline{(\mathcal{B})}}} \delta(\rho)^{\overrightarrow{(\mathcal{B})}}+J \frac{\partial \check{W}^{\text {cap }}}{\partial(\beta)^{\overline{(\mathcal{B})}}} \delta(\beta)^{\overrightarrow{(\mathcal{B})}}\right) \\
& =\left(\check{W}^{\text {cap }} \frac{\partial J}{\partial F} \delta F+J \frac{\partial \check{W}^{\text {cap }}}{\partial(\rho)^{\overline{(\mathcal{B})}}} \frac{\partial(\rho)^{\overrightarrow{\mathcal{B}}}}{\partial F} \delta F\right)+\frac{\partial \check{W}^{\text {cap }}}{\partial(\beta)^{\overline{(\mathcal{B})}}} J\left(\frac{\partial(\beta)^{\overrightarrow{\mathcal{B}})}}{\partial F} \delta F+\frac{\partial(\beta)^{\overrightarrow{\mathcal{B}})}}{\partial \nabla F} \delta \nabla F\right)
\end{aligned}
$$

As a consequence (with another abuse of notation) we have

$$
\begin{aligned}
\frac{\partial W^{\text {cap }}}{\partial F} & =\check{W}^{\text {cap }} \frac{\partial J}{\partial F}+J \frac{\partial \check{W}^{\text {cap }}}{\partial \rho} \frac{\partial \rho}{\partial F}+J \frac{\partial \check{W}^{\text {cap }}}{\partial \beta} \frac{\partial \beta}{\partial F} \\
\frac{\partial W^{\text {cap }}}{\partial \nabla F} & =J \frac{\partial \check{W}^{\text {cap }}}{\partial \beta} \frac{\partial \beta}{\partial \nabla F}
\end{aligned}
$$

\subsubsection{Eulerian balance equations for capillary fluids}

Following the original methods introduced by Piola, after having applied the principle of least action or the principle of virtual work in the Lagrangian description, we must transform the obtained stationarity conditions into some other conditions which are valid in the Eulerian description. As previously seen, in Lagrangian description the balance equations for capillary fluids read

$$
-\frac{\partial}{\partial t}\left(\rho_{0} v_{i}\right)+D I V_{X}\left(\frac{\partial W^{\text {eul }}}{\partial F}+\frac{\partial W^{\text {cap }}}{\partial F}\right)-D I V_{X}\left(D I V_{X}\left(\frac{\partial W^{\text {cap }}}{\partial \nabla F}\right)\right)=0
$$

where $W^{\text {eul }}$ and $W^{\text {cap }}$ were defined, respectively, in (9) and (26). The terms in (29), which are specific to capillary fluids, must therefore be estimated. Starting from equation (27) and using the following result (calculated in (8.1.7) and (8.1.2))

$$
\frac{\partial J}{\partial F_{M}^{i}}=J\left(F^{-1}\right)_{i}^{M}, \quad \frac{\partial \rho}{\partial F_{M}^{i}}=-\rho\left(F^{-1}\right)_{i}^{M}, \quad \frac{\partial \beta}{\partial F_{M}^{i}}=-2 g^{a b}\left(\rho_{, a} \rho_{, i}\left(F^{-1}\right)_{b}^{M}+\rho_{, a}\left(\rho\left(F^{-1}\right)_{i}^{M}\right)_{, b}\right)
$$

we obtain (the notation $(\cdot)^{\overrightarrow{\mathcal{B}})}$ has been dropped to yield more readable formulas),

$$
\frac{\partial W^{\text {cap }}}{\partial F_{M}^{i}}=-\mathcal{P}^{\text {cap }} J\left(F^{-1}\right)_{i}^{M}-2 \frac{\partial \check{W}^{\text {cap }}}{\partial \beta} J\left(g^{a b} \rho_{, a} \rho, i\left(F^{-1}\right)_{b}^{M}+\beta\left(F^{-1}\right)_{i}^{M}+g^{a b} \rho_{, a} \rho\left(F^{-1}\right)_{i, b}^{M}\right)
$$

where we have introduced

$$
\mathcal{P}^{\text {cap }}:=\rho \frac{\partial \breve{W}^{\text {cap }}}{\partial \rho}-\check{W}^{\text {cap }}
$$

\subsubsection{Piola stress decomposition}

In the remaining part of the paper, different Piola stress tensors will be considered. Therefore, and in order to avoid any misunderstanding, some time will be devoted to properly define these different stress tensors. This discussion is specific to higher-order continua, since for the first gradient continuum these different tensors are either identical or null. As a starting point we define the bulk Piola stress for capillary fluids by

$$
\mathbb{P}_{i}^{M}:=\frac{\partial W^{\text {eul }}}{\partial F_{M}^{i}}+\frac{\partial W^{\text {cap }}}{\partial F_{M}^{i}}-\frac{\partial}{\partial X^{A}}\left(\frac{\partial W^{\text {cap }}}{\partial F_{M, A}^{i}}\right)
$$

as the quantity that appears in the Lagrangian balance equation

$$
-\frac{\partial}{\partial t}\left(\rho_{0} v_{i}\right)+\frac{\partial \mathbb{P}_{i}^{A}}{\partial X^{A}}-\frac{\partial U^{\mathrm{ext}}}{\partial \chi^{i}}=0
$$

This tensor is an effective tensor (in the following effective tensor are written using blackboard fonts) since it is composed of tensors of different order, as

$$
\mathbb{P}_{i}^{M}:=\mathrm{P}_{i}^{M}+\frac{\partial}{\partial X^{A}}\left(\mathrm{H}_{i}^{M A}\right),
$$

where $\mathrm{P}_{i}^{M}$ is the classical Piola stress, and $\mathrm{H}_{i}^{M A}$ is third-order Hyper Piola stress defined as 


$$
\mathrm{H}_{i}^{M N}:=\frac{\partial W}{\partial F_{M, N}^{i}} .
$$

It is worth noting that for capillary fluids, the classical Piola stress can be decomposed as

$$
\mathrm{P}_{i}^{M}:=\left(\mathrm{P}^{\mathrm{eul}}\right)_{i}^{M}+\left(\mathrm{P}^{\mathrm{cal}}\right)_{i}^{M}
$$

Hence, another effective tensor can be defined

$$
\left(\mathbb{P}^{\mathrm{cal}}\right)_{i}^{M}:=\left(\mathrm{P}^{\mathrm{cal}}\right)_{i}^{M}+\frac{\partial}{\partial X^{A}}\left(\mathrm{H}_{i}^{M A}\right)
$$

resulting in the following additive decomposition of the bulk Piola stress

$$
\mathbb{P}_{i}^{M}:=\left(\mathrm{P}^{\mathrm{eul}}\right)_{i}^{M}+\left(\mathbb{P}^{\mathrm{cal}}\right)_{i}^{M}
$$

\subsubsection{Piola stress for capillary fluids}

Now we will effectively compute the effective bulk Piola tensor. To that aim, we start by calculating the relevant term by using (28) and

(56) thus,

$$
\begin{gathered}
\frac{\partial \beta}{\partial F_{M, N}^{i}}=-2 g^{a b} \rho \rho_{, a}\left(F^{-1}\right)_{i}^{M}\left(F^{-1}\right)_{b}^{N}, \\
-\frac{\partial}{\partial X^{A}}\left(\frac{\partial \check{W}^{\mathrm{cap}}}{\partial F_{M, A}^{i}}\right)=-\frac{\partial}{\partial X^{A}}\left(J \frac{\partial \check{W}^{\text {cap }}}{\partial \beta} \frac{\partial \beta}{\partial F_{M, A}^{i}}\right)=\frac{\partial}{\partial X^{A}}\left(\rho_{0} 2 g^{a b} \rho_{, a} \frac{\partial \check{W}^{\mathrm{cap}}}{\partial \beta}\left(F^{-1}\right)_{i}^{M}\left(F^{-1}\right)_{b}^{A}\right) \\
=\frac{\partial}{\partial X^{A}}\left(\rho_{0} 2 g^{a b} \rho_{, a} \frac{\partial \check{W}^{\mathrm{cap}}}{\partial \beta}\left(F^{-1}\right)_{i}^{M}\right)\left(F^{-1}\right)_{b}^{A}+\rho_{0} 2 g^{a b} \rho_{, a} \frac{\partial \check{W}^{\mathrm{cap}}}{\partial \beta}\left(F^{-1}\right)_{i}^{M} \frac{\partial}{\partial X^{A}}\left(\left(F^{-1}\right)_{b}^{A}\right) \\
=\frac{\partial}{\partial x^{b}}\left(\rho_{0} 2 g^{a b} \rho_{, a} \frac{\partial \check{W}^{\text {cap }}}{\partial \beta}\left(F^{-1}\right)_{i}^{M}\right)+\rho_{0} 2 g^{a b} \rho_{, a} \frac{\partial \check{W}^{\mathrm{cap}}}{\partial \beta}\left(F^{-1}\right)_{i}^{M}\left(F^{-1}\right)_{b, A}^{A} .
\end{gathered}
$$

Now we use (42), i.e.

$$
\left(F^{-1}\right)_{i, A}^{A}=\frac{\rho_{, A}}{\rho}\left(F^{-1}\right)_{i}^{A}=\frac{\rho_{, i}}{\rho}
$$

to derive

$$
-\frac{\partial}{\partial X^{A}}\left(\frac{\partial W^{\text {cap }}}{\partial F_{M, A}^{i}}\right)=\frac{\partial}{\partial x^{b}}\left(2 \frac{\partial \check{W}^{\text {cap }}}{\partial \beta} \rho_{0} g^{a b} \rho_{, a}\right)\left(F^{-1}\right)_{i}^{M}+2 \frac{\partial \check{W}^{\text {cap }}}{\partial \beta} \rho_{0} g^{a b} \rho_{, a}\left(F^{-1}\right)_{i, b}^{M}+2 \beta \frac{\partial \check{W}^{\text {cap }}}{\partial \beta} J\left(F^{-1}\right)_{i}^{M} .
$$

Using (30) and (32) in (31) we obtain

$$
\mathbb{P}_{i}^{M}=\left(-\left(p(\rho)+\mathcal{P}^{\text {cap }}\right)+\rho \frac{\partial}{\partial x^{b}}\left(2 \frac{\partial \check{W}^{\text {cap }}}{\partial \beta} g^{a b} \rho_{, a}\right)\right) J\left(F^{-1}\right)_{i}^{M}-2 \frac{\partial \check{W}^{\text {cap }}}{\partial \beta} g^{a b} \rho_{, a} \rho_{, i} J\left(F^{-1}\right)_{b}^{M}
$$

where we have used

$$
\frac{\partial W^{\mathrm{eul}}}{\partial F_{M}^{i}}=-J p(\rho)\left(F^{-1}\right)_{i}^{M}
$$

\subsubsection{Cauchy stress for capillary fluids}

As for the effective bulk Piola stress, we define the effective bulk Cauchy stress as the quantity that appears in the Eulerian balance equation

$$
-\rho\left(\frac{\partial v_{i}^{\overrightarrow{\mathcal{E}})}}{\partial t}+\frac{\partial v_{i}^{\overrightarrow{\mathcal{E}})}}{\partial x^{a}}\left(v^{a}\right)^{\overrightarrow{\mathcal{E}})}\right)-\frac{\partial}{\partial x^{b}}\left(\mathbb{T}_{i}^{b}\right)-\rho\left(\frac{\partial\left(U^{\left.\exp / \rho_{0}\right)}\right.}{\partial \chi^{i}}\right)^{\overrightarrow{\mathcal{E})}}=0 .
$$

This effective tensor can be decomposed as

$$
\mathbb{T}_{i}^{j}:=\mathrm{T}_{i}^{j}+\frac{\partial}{\partial x^{A}}\left(\mathrm{~S}_{i}^{j a}\right),
$$

where $\mathrm{T}_{i}^{j}$ is the second-order capillary Cauchy stress, and $\mathrm{S}_{i}^{j k}$ is the third-order capillary Hyper Cauchy stress. As previously explained, the second-order Cauchy stress can be decomposed as 


$$
\mathrm{T}_{i}^{j}:=\left(\mathrm{T}^{\mathrm{eul}}\right)_{i}^{j}+\left(\mathrm{T}^{\mathrm{cap}}\right)_{i}^{j}
$$

Hence, another effective tensor can be defined

$$
\left(\mathbb{T}^{\mathrm{cap}}\right)_{i}^{j}:=\left(\mathrm{T}^{\mathrm{cap}}\right)_{i}^{j}+\frac{\partial}{\partial x^{a}}\left(\mathrm{~S}_{i}^{j a}\right),
$$

resulting in the following additive decomposition of the following bulk Cauchy stress:

$$
\mathbb{T}_{i}^{j}:=\left(\mathrm{T}^{\mathrm{eul}}\right)_{i}^{j}+\left(\mathbb{T}^{\mathrm{cap}}\right)_{i}^{j}
$$

Let us now return to the explicit determination of $\mathbb{T}_{i}^{j}$. By recalling (see Appendix A) the Piola transformation of tensors from the Lagrangian to the Eulerian description, i.e.

$$
\mathbb{T}_{i}^{j}=J^{-1}\left(\mathbb{P}_{i}^{A} F_{A}^{j}\right)^{\overrightarrow{(\mathcal{E})}},
$$

the bulk Cauchy stress tensor for capillary fluids is obtained as

$$
\mathbb{T}_{i}^{j}=\left(-\left(p(\rho)+\mathcal{P}^{\text {cap }}\right)+\rho \frac{\partial}{\partial x^{b}}\left(2 \frac{\partial \check{W}^{\text {cap }}}{\partial \beta} g^{a b} \rho_{, a}\right)\right) \delta_{i}^{j}-2 \frac{\partial \check{W}^{\text {cap }}}{\partial \beta} g^{a j} \rho_{, a} \rho_{, i} .
$$

In the case of Cahn-Hilliard fluids with a constant $\lambda$ we have

$$
2 \frac{\partial \check{W}^{\text {cap }}}{\partial \beta}=\lambda, \quad \check{W}^{\text {cap }}=-\mathcal{P}^{\text {cap }}=\frac{\lambda}{2} g^{a b} \rho_{, a} \rho_{, b}
$$

so that

$$
\mathbb{T}_{i}^{j}=\left(-p(\rho)+\frac{\lambda}{2} g^{a b} \rho_{, a} \rho_{, b}+\rho \frac{\partial}{\partial x^{b}}\left(\lambda g^{a b} \rho_{, a}\right)\right) \delta_{i}^{j}-\lambda g^{a j} \rho_{, a} \rho_{, i},
$$

which is exactly the result found in the literature (see Seppecher [143] or Casal and Gouin [26, 27]). Let us now develop the Eulerian divergence of the effective capillary Cauchy tensor:

$$
\begin{aligned}
\frac{\partial}{\partial x^{c}}\left(\mathbb{T}_{i}^{c}\right) & =\frac{\partial}{\partial x^{c}}\left(\left(-p(\rho)+\frac{\lambda}{2} g^{a b} \rho_{, a} \rho_{, b}+\rho \frac{\partial}{\partial x^{b}}\left(\lambda g^{a b} \rho_{, a}\right)\right) \delta_{i}^{c}-\lambda g^{a c} \rho_{, a} \rho_{, i}\right) \\
& =-\frac{\partial}{\partial x^{i}} p(\rho)+\lambda g^{a b} \rho_{, a} \rho_{, b i}+\frac{\partial}{\partial x^{i}}\left(\rho \lambda g^{a b} \rho_{, a b}\right)-\lambda g^{a c} \rho_{, a c} \rho_{, i}-\lambda g^{a c} \rho_{, a} \rho_{, i c} \\
& =-\frac{\partial}{\partial x^{i}} p(\rho)+\lambda \rho \frac{\partial}{\partial x^{i}}\left(g^{a b} \rho_{, a b}\right) .
\end{aligned}
$$

In conclusion the Eulerian balance equation for Cahn-Hilliard fluids is:

$$
-\rho\left(\frac{\partial v_{i}^{\overrightarrow{\mathcal{E}}}}{\partial t}+\frac{\partial v_{i}^{(\overrightarrow{\mathcal{E}})}}{\partial x^{a}}\left(v^{a}\right)^{\overrightarrow{\mathcal{E}})}\right)-\frac{\partial}{\partial x^{i}} p(\rho)+\lambda \rho \frac{\partial}{\partial x^{i}}\left(g^{a b} \rho_{, a b}\right)-\rho\left(\frac{\partial\left(U^{e x p} / \rho_{0}\right)}{\partial \chi^{i}}\right)^{\overrightarrow{\mathcal{E}})}=0 .
$$

To complete the description of the model, the associated boundary conditions have to be supplied.

\subsubsection{Boundary terms}

In the particular case of capillary fluids the Hyper Piola tensor has the following explicit expression:

$$
\mathrm{H}_{i}^{M N}=\frac{\partial W^{\text {cap }}}{\partial \beta} \frac{\partial \beta}{\partial F_{M, N}^{i}}=-\lambda \rho_{0} \rho_{, a} g^{a b}\left(F^{-1}\right)_{b}^{M}\left(F^{-1}\right)_{i}^{N} .
$$

Its Eulerian equivalent is the following Hyper Cauchy tensor

$$
\mathrm{S}_{i}^{j k}=-J^{-1} H_{i}^{A B} F_{B}^{j} F_{A}^{k}=-J^{-1} \rho_{0} g^{a b} \rho_{, a} \lambda\left(F^{-1}\right)_{i}^{B}\left(F^{-1}\right)_{b}^{A} F_{B}^{j} F_{A}^{k}=-\lambda \rho g^{a k} \rho_{, a} \delta_{i}^{j} .
$$

Double force The expression of contact double force will be discussed first. In the absence of surface external double force, the boundary conditions read

$$
\frac{\partial W^{\text {cap }}}{\partial \nabla F} \cdot(N \otimes N)=0
$$


or, in components

$$
\frac{\partial W^{\text {cap }}}{\partial F_{A, B}^{i}} N_{A} N_{B}=-\lambda \rho_{0} \rho_{, a} g^{a b}\left(F^{-1}\right)_{b}^{A}\left(F^{-1}\right)_{i}^{B} N_{A} N_{B}=0
$$

Using the Piola transformation for normals (43), the former expression is rewritten

$$
-\lambda \rho_{0} \rho_{, a} g^{a b}\left(F^{-1}\right)_{b}^{M}\left(F^{-1}\right)_{i}^{N} J^{-1} F_{M}^{c} n_{c} J^{-1} F_{N}^{e} n_{e}=0
$$

Hence, for line forces (23) we obtain

$$
-J^{-1} \lambda \rho \rho_{, a} g^{a b} n_{b} \nu_{i}=0 .
$$

Force In the absence of external force, the new boundary conditions read

$$
\mathbb{P}_{i}^{A} N_{A}+\frac{\partial}{\partial X^{E}}\left(P_{C}^{D}\left(\mathrm{H}_{i}^{B C} N_{B}\right)\right) P_{D}^{E}=0,
$$

or, using the Piola transformation, in Eulerian form

$$
\mathbb{T}_{i}^{a} n_{a}+\frac{\partial}{\partial x^{e}}\left(P_{c}^{d}\left(\mathrm{~S}_{i}^{b c} n_{b}\right)\right) P_{d}^{e}=0
$$

The first term will first be considered. This term can be expanded as

$$
\begin{aligned}
\mathbb{T}_{i}^{a} n_{a} & =\left[\left(-p(\rho)+\frac{\lambda}{2} g^{b c} \rho_{, b} \rho_{, c}+\rho \frac{\partial}{\partial x^{c}}\left(\lambda g^{b c} \rho_{, b}\right)\right) \delta_{i}^{a}-\lambda g^{b a} \rho_{, b} \rho_{, i}\right] n_{a} \\
& =\left(-p(\rho)+\frac{\lambda}{2} g^{b c} \rho_{, b} \rho_{, c}+\rho \frac{\partial}{\partial x^{c}}\left(\lambda g^{b c} \rho_{, b}\right)\right) n_{i}-\lambda g^{a b} \rho_{, b} \rho_{, i} n_{a} \\
& =\left(-p(\rho)+\frac{\lambda}{2} g^{b c} \rho_{, b} \rho_{, c}+\rho \frac{\partial}{\partial x^{c}}\left(\lambda g^{b c} \rho_{, b}\right)\right) n_{i}-\lambda n^{b} \rho_{, b} \rho_{, i} \\
& =\left(-p(\rho)+\frac{\lambda}{2} g^{b c} \rho_{, b} \rho_{, c}+\rho \frac{\partial}{\partial x^{c}}\left(\lambda g^{b c} \rho_{, b}\right)-\lambda n^{i} \rho_{, i} n^{b} \rho_{, b}\right) n_{i} .
\end{aligned}
$$

It remains now to consider the last part of the boundary conditions, i.e.

$$
\frac{\partial}{\partial x^{d}}\left(P_{b}^{c}\left(\mathrm{~S}_{i}^{a b} n_{a}\right)\right) P_{c}^{d}
$$

This computation is a bit more tricky. In order to easily derive the expression, the following identities will be established:

$$
\begin{aligned}
& Q \cdot(v \otimes n)=(v \cdot n) Q \\
& P \cdot(v \otimes n)=(v \cdot n) P
\end{aligned}
$$

Their demonstration is straightforward:

$$
\begin{aligned}
Q_{a}^{i} v^{a} n_{j} & =n^{i} n_{a} v^{a} n_{j}=n_{a} v^{a} n^{i} n_{j}=Q_{j}^{i} v^{a} n_{a} \\
P_{a}^{i} v^{a} n_{j} & =\left(\delta_{a}^{i}-Q_{a}^{i}\right) v^{a} n_{j}=\left(\delta_{j}^{i} \delta_{a}^{j} v^{a} n_{j}\right)+Q_{j}^{i} v^{a} n_{a} \\
& =\delta_{j}^{i} v^{a} n_{a}+Q_{j}^{i} v^{a} n_{a}=\left(\delta_{j}^{i}+Q_{j}^{i}\right) v^{a} n_{a} \\
& =P_{j}^{i} v^{a} n_{a}
\end{aligned}
$$

Now, using the definition of the hyperstress for capillary fluids, we obtain

$$
\mathrm{S}_{i}^{a j} n_{a}=-\lambda \rho \rho^{j} n_{i}
$$

In the following, the factor $-\lambda \rho$ will dropped and only added at the end. Using the identity (33) we have the first transformation relation

$$
P_{a}^{i}\left(\rho^{a} n_{j}\right)=\rho^{a} n_{a} P_{j}^{i} .
$$




$$
\nabla_{k}^{S}\left(P_{a}^{i}\left(\rho^{a} n_{j}\right)\right)=\nabla_{k}^{S}\left(\rho^{a} n_{a} P_{j}^{i}\right)=\nabla_{k}^{S}\left(\rho^{a} n_{a}\right) P_{j}^{i}+\rho^{a} n_{a} \nabla_{k}^{S}\left(P_{j}^{i}\right)
$$

where $\nabla_{k}^{S}:=P_{k}^{a} \frac{\partial}{\partial x_{a}}$ denotes the surface (tangential) gradient. Let us now compute the surface gradient of the projection operator $P$,

$$
\begin{aligned}
\nabla_{k}^{S}\left(P_{j}^{i}\right) & =\nabla_{k}^{S} \delta_{j}^{i}-\nabla_{k}^{S}\left(n^{i} n_{j}\right)=-\left(\nabla_{k}^{S}\left(n^{i}\right) n_{j}+n^{i} \nabla_{k}^{S}\left(n_{j}\right)\right) \\
& =L_{k}^{i} n_{j}+n^{i} L_{k j}
\end{aligned}
$$

where $L_{i j}:=-P_{i}^{a} n_{a j}$ is the Weingarten curvature tensor. Therefore, it follows that

$$
\nabla_{k}^{S}\left(P_{a}^{i}\left(\rho^{a} n_{j}\right)\right)=\nabla_{k}^{S}\left(\rho^{a} n_{a}\right) P_{j}^{i}+\rho^{a} n_{a}\left(L_{k}^{i} n_{j}+n^{i} L_{k j}\right)
$$

To obtain the surface divergence it remains to multiply the previous result by $\delta_{k}^{i}$

$$
\nabla_{i}^{S}\left(P_{a}^{i}\left(\rho^{a} n_{j}\right)\right)=\nabla_{i}^{S}\left(\rho^{a} n_{a}\right) P_{j}^{i}+\rho^{a} n_{a}\left(L_{i}^{i} n_{j}+n^{i} L_{i j}\right) .
$$

This expression can be simplified, using

$$
\begin{gathered}
\nabla_{i}^{S} P_{j}^{i}=P_{i}^{a} \frac{\partial}{\partial x_{a}} P_{j}^{i}=P_{i}^{a} P_{j}^{i} \frac{\partial}{\partial x_{a}}=\nabla_{j}^{S} \\
n^{i} L_{i j}=n^{i} P_{i}^{a} n_{a j}=0
\end{gathered}
$$

and

$$
2 H:=L_{i}^{i}
$$

where $H$ is the surface mean curvature. Therefore, at the end of the journey,

$$
\nabla_{i}^{S}\left(P_{a}^{i}\left(\rho^{a} n_{j}\right)\right)=\nabla_{j}^{S}\left(\rho^{a} n_{a}\right)+2 \rho^{a} n_{a} H n_{j} .
$$

Once the two parts added, we obtain

$$
\begin{gathered}
\left(-p(\rho)+\frac{\lambda}{2} g^{a b} \rho_{, a} \rho_{, b}+\rho \frac{\partial}{\partial x^{b}}\left(\lambda g^{a b} \rho_{, a}\right)-\lambda n^{i} \rho_{, i} n^{a} \rho_{, a}+2 \rho^{a} n_{a} H\right) n_{i}+\nabla_{i}^{S}\left(\rho^{a} n_{a}\right)=0, \\
-p^{*} n_{i}+\nabla_{i}^{S}\left(\rho^{a} n_{a}\right)=0,
\end{gathered}
$$

in which

$$
p^{*}=\left(p(\rho)-\frac{\lambda}{2} g^{a b} \rho_{, a} \rho_{, b}-\rho \frac{\partial}{\partial x^{b}}\left(\lambda g^{a b} \rho_{, a}\right)+\lambda n^{i} \rho_{, i} n^{a} \rho_{, a}+2 \rho^{a} n_{a} H\right) .
$$

This is exactly the result found in $[143,144,26,27]$.

\subsubsection{Bernoulli Law for capillary fluids}

The results in the previous sections imply that for capillary fluids the following Eulerian Balance of force holds (see also [25, 26])

$$
-\rho\left(\frac{\partial v_{i}^{\overrightarrow{\mathcal{E}})}}{\partial t}+\frac{\partial v_{i}^{(\overrightarrow{\mathcal{E}})}}{\partial x^{a}}\left(v^{a}\right)^{\overrightarrow{\mathcal{E}})}\right)-\frac{\partial}{\partial x^{i}}(p(\rho))+\frac{\partial}{\partial x^{b}}\left(\mathbb{T}^{\mathrm{cap}}\right)_{i}^{b}-\rho\left(\frac{\partial U / \rho_{0}}{\partial \chi^{i}}\right)^{\overrightarrow{(\mathcal{E})}}=0
$$

where we have introduced the constitutive equations

$$
\begin{gathered}
\left(\mathbb{T}^{\text {cap }}\right)_{i}^{b}=\left(-\mathcal{P}^{\text {cap }}(\rho, \beta)+\rho \frac{\partial}{\partial x^{b}}\left(2 \frac{\partial \check{W}^{\text {cap }}}{\partial \beta} g^{a b} \rho_{, a}\right)\right) \delta_{i}^{j}-2 \frac{\partial \check{W}^{\text {cap }}}{\partial \beta} g^{a j} \rho_{, a} \rho_{, i}, \\
-\mathcal{P}^{\text {cap }}:=\check{W}^{\text {cap }}-\rho \frac{\partial \check{W}^{\text {cap }}}{\partial \rho} \quad ; \quad p(\rho):=\rho^{2} \frac{\partial\left(\Psi / \rho_{0}\right)}{\partial \rho} .
\end{gathered}
$$


If the last relationship is invertible one can express the density as a function $\hat{\rho}$ of the pressure and introduce the function

$$
Q(p)=\Delta \frac{1}{\hat{\rho}(p)} d p
$$

which has the remarkable property

$$
\frac{\partial Q(p)}{\partial x^{i}}=\frac{1}{\hat{\rho}(p)} \frac{\partial p}{\partial x^{i}}
$$

As a consequence, once divided by $\rho$ the equations become

$$
-\frac{\partial v_{i}^{\overrightarrow{\mathcal{E}})}}{\partial t}-\frac{\partial v_{i}^{(\overrightarrow{\mathcal{E}})}}{\partial x^{a}}\left(v^{a}\right)^{\overrightarrow{(\mathcal{E})}}-\frac{\partial}{\partial x^{i}}(Q(p))+\frac{1}{\rho} \frac{\partial}{\partial x^{b}}\left(\mathbb{T}^{\mathrm{cap}}\right)_{i}^{b}-\left(\frac{\partial U / \rho_{0}}{\partial \chi^{i}}\right)^{\overrightarrow{(\mathcal{E})}}=0 .
$$

The calculation of $\frac{\partial}{\partial x^{a}}\left(\mathbb{T}^{\text {cap }}\right)_{i}^{a}$ We have to compute the following term

$$
\begin{aligned}
& \frac{\partial}{\partial x^{a}}\left(\mathbb{T}^{\text {cap }}\right)_{i}^{a}=\frac{\partial}{\partial x^{a}}\left(\left(-\mathcal{P}^{\text {cap }}(\rho, \beta)+\rho \frac{\partial}{\partial x^{b}}\left(2 \frac{\partial \check{W}^{\text {cap }}}{\partial \beta} g^{b c} \rho_{, c}\right)\right) \delta_{i}^{a}-2 \frac{\partial \check{W}^{\text {cap }}}{\partial \beta} g^{d a} \rho_{, d} \rho_{, i}\right) \\
& =\underbrace{\frac{\partial}{\partial x^{i}}\left(\check{W}^{\text {cap }}-\rho \frac{\partial \check{W}^{\text {cap }}}{\partial \rho}+\rho \frac{\partial}{\partial x^{b}}\left(2 \frac{\partial \check{W}^{\text {cap }}}{\partial \beta} g^{b c} \rho, c\right)\right)}_{A} \underbrace{-2 \frac{\partial}{\partial x^{a}}\left(\frac{\partial \check{W}^{\text {cap }}}{\partial \beta} g^{d a} \rho_{, d} \rho_{, i}\right)}_{B} .
\end{aligned}
$$

Let us process first the term labeled $A$ :

$$
\begin{aligned}
A & =\frac{\partial}{\partial x^{i}} \check{W}^{\text {cap }}-\frac{\partial}{\partial x^{i}}\left(\rho \frac{\partial \check{W}^{\text {cap }}}{\partial \rho}\right)+\frac{\partial}{\partial x^{i}}\left(\rho \frac{\partial}{\partial x^{b}}\left(2 \frac{\partial \check{W}^{\text {cap }}}{\partial \beta} g^{b c} \rho_{, c}\right)\right) \\
& =\frac{\partial \check{W}^{\text {cap }}}{\partial \rho} \frac{\partial \rho}{\partial x^{i}}+\frac{\partial \check{W}^{\text {cap }}}{\partial \beta} \frac{\partial \beta}{\partial x^{i}}-\rho_{, i} \frac{\partial \check{W}^{\text {cap }}}{\partial \rho}-\rho \frac{\partial}{\partial x^{i}}\left(\frac{\partial \check{W}^{\text {cap }}}{\partial \rho}\right)+\frac{\partial}{\partial x^{i}}\left(\rho \frac{\partial}{\partial x^{b}}\left(2 \frac{\partial \check{W}^{\text {cap }}}{\partial \beta} g^{b c} \rho_{, c}\right)\right) \\
& =\frac{\partial \check{W}^{\text {cap }}}{\partial \beta} \frac{\partial \beta}{\partial x^{i}}-\rho \frac{\partial}{\partial x^{i}}\left(\frac{\partial \check{W}^{\text {cap }}}{\partial \rho}\right)+\rho_{, i} \frac{\partial}{\partial x^{b}}\left(2 \frac{\partial \check{W}^{\text {cap }}}{\partial \beta} g^{b c} \rho_{, c}\right)+\left(\rho \frac{\partial}{\partial x^{i}} \frac{\partial}{\partial x^{b}}\left(2 \frac{\partial \check{W}^{\text {cap }}}{\partial \beta} g^{b c} \rho_{, c}\right)\right) .
\end{aligned}
$$

The term $B$ is easy to determine:

$$
B=-2 \rho_{, i} \frac{\partial}{\partial x^{a}}\left(\frac{\partial \check{W}^{\text {cap }}}{\partial \beta} g^{a d} \rho_{, d}\right)-2 \frac{\partial \check{W}^{\text {cap }}}{\partial \beta} g^{d a} \rho_{, d} \rho_{, i a}
$$

Therefore we have

$$
\frac{\partial}{\partial x^{a}}\left(\mathbb{T}^{\text {cap }}\right)_{i}^{a}=-\rho \frac{\partial}{\partial x^{i}}\left(\frac{\partial \check{W}^{\text {cap }}}{\partial \rho}\right)+\left(\rho \frac{\partial}{\partial x^{i}} \frac{\partial}{\partial x^{b}}\left(2 \frac{\partial \check{W}^{\text {cap }}}{\partial \beta} g^{b c} \rho_{, c}\right)\right)+\frac{\partial \check{W}^{\text {cap }}}{\partial \beta} \frac{\partial \beta}{\partial x^{i}}-2 \frac{\partial \check{W}^{\text {cap }}}{\partial \beta} g^{d a} \rho_{, d} \rho_{, i a} \quad,
$$

and recalling that

$$
\frac{\partial \beta}{\partial x^{i}}=\frac{\partial}{\partial x^{i}}\left(g^{a b} \rho_{, a} \rho_{, b}\right)=2 g^{a b} \rho_{, a} \rho_{, b i}
$$

the desired result is finally obtained

$$
\begin{aligned}
\frac{\partial}{\partial x^{a}}\left(\mathbb{T}^{\mathrm{cap}}\right)_{i}^{a} & =-\rho \frac{\partial}{\partial x^{i}}\left(\frac{\partial \check{W}^{\text {cap }}}{\partial \rho}\right)+\rho \frac{\partial}{\partial x^{i}} \frac{\partial}{\partial x^{b}}\left(2 \frac{\partial \check{W}^{\text {cap }}}{\partial \beta} g^{b c} \rho_{, c}\right) \\
& =\rho \frac{\partial}{\partial x^{i}}\left(\frac{\partial}{\partial x^{b}}\left(2 \frac{\partial \check{W}^{\text {cap }}}{\partial \beta} g^{b c} \rho_{, c}\right)-\left(\frac{\partial \check{W}^{\text {cap }}}{\partial \rho}\right)\right) \\
& =\rho \frac{\partial}{\partial x^{i}}\left(\mathcal{P}^{\mathrm{eff}}\left(\rho ; \rho_{, a} ; g^{a b} \rho_{, a b}\right)\right) .
\end{aligned}
$$

\subsubsection{Bernoulli constant of motion along flow curves}

To conclude our argument we need a last tensorial equality (see e.g. Lebedev et al. [88])

$$
\frac{\partial v_{i}}{\partial x^{a}} v^{a}=\frac{\partial v^{a}}{\partial x^{i}} v_{a}+\left(\frac{\partial v_{i}}{\partial x^{a}} v^{a}-\frac{\partial v^{a}}{\partial x^{i}} v_{a}\right)=\frac{\partial}{\partial x^{i}}\left(\frac{1}{2} v^{a} v_{a}\right)+W_{i}^{a} v_{a}
$$

where the tensor $W_{i}^{j}$ defined by

$$
W_{i}^{j}:=\frac{\partial v_{i}}{\partial x^{j}}-\frac{\partial v^{j}}{\partial x^{i}}
$$


clearly saisfies the equality

$$
W_{b}^{a} v_{a} v^{b}=\left(\frac{\partial v_{b}}{\partial x^{a}} v^{b} v^{a}-\frac{\partial v^{a}}{\partial x^{b}} v^{b} v_{a}\right)=\frac{1}{2}\left(\frac{\partial\left(v_{b} v^{b}\right)}{\partial x^{a}} v^{a}-\frac{\partial\left(v^{a} v_{a}\right)}{\partial x^{b}} v^{b}\right)=0 .
$$

Let consider the equations (34)

$$
-\frac{\partial v_{i}^{(\mathcal{E})}}{\partial t}-\frac{\partial v_{i}^{\overrightarrow{\mathcal{E}}}}{\partial x^{a}}\left(v^{a}\right)^{\overrightarrow{\mathcal{E}})}-\frac{\partial}{\partial x^{i}}(Q(p))+\frac{1}{\rho} \frac{\partial}{\partial x^{b}}\left(\mathbb{S}_{i}^{b}\right)-\left(\frac{\partial U / \rho_{0}}{\partial \chi^{i}}\right)^{\overrightarrow{\mathcal{E}})}=0 .
$$

If the applied bulk external forces are such that there exists a scalar Eulerian function $V$ for which

$$
\left(\frac{\partial U / \rho_{0}}{\partial \chi^{i}}\right)^{\overrightarrow{\mathcal{E}})}=\frac{\partial V}{\partial x^{i}}
$$

and by making use of $(35)$ ( the notation $(\cdot)^{\overrightarrow{(\mathcal{E})}}$ has been dropped), we obtain

$$
-\frac{\partial v_{i}}{\partial t}-\frac{\partial}{\partial x^{i}}\left(\frac{1}{2} v^{c} v_{c}\right)+W_{i}^{d} v_{d}-\frac{\partial}{\partial x^{i}}(Q(p))+\frac{\partial}{\partial x^{i}}\left(\frac{\partial}{\partial x^{b}}\left(2 \frac{\partial \check{W}^{\text {cap }}}{\partial \beta} g^{a b} \rho_{, a}\right)-\left(\frac{\partial \check{W}^{\text {cap }}}{\partial \rho}\right)\right)-\frac{\partial V}{\partial x^{i}}=0 .
$$

By calculating the inner product with $v$ we get

$$
\frac{\partial}{\partial t}\left(\frac{1}{2} v \cdot v\right)+\nabla\left(\frac{1}{2} v \cdot v+Q(p(\rho))-\mathcal{P}^{\mathrm{eff}}\left(\rho ; \rho_{, a} ; g^{a b} \rho_{, a b}\right)+V\right) \cdot v=0
$$

and if the field $v$ be stationary, i.e. if

$$
\frac{\partial v}{\partial t}=0
$$

the last equation becomes

$$
\nabla\left(\frac{1}{2} v \cdot v+Q(p(\rho))-\mathcal{P}^{\mathrm{eff}}\left(\rho ; \rho_{, a} ; g^{a b} \rho_{, a b}\right)+V\right) \cdot v=0
$$

i.e. along (steady) flow curves there exists a constant $K_{0}$ such that

$$
\frac{1}{2} v \cdot v+Q(p(\rho))-\mathcal{P}^{\mathrm{eff}}\left(\rho ; \rho_{, a} ; g^{a b} \rho_{, a b}\right)+V=K_{0}
$$

\section{Conclusions: towards continuum analytical mechanics ?}

The role of the principle of least action (or of its weaker version the principle of virtual work) in applied mathematics, and in particular in mathematical physics, has been controversial since its very first formulations. The attitude towards this postulation is often one of total rejection. Indeed, both the supporters of variational postulations and the supporters of balance of everything behave often as if the controversy does not exist. They simply pretend that the other postulation process is not used at all or anymore. Of course the supporters of balance of everything are aware of the importance of a variational principle, especially when a numerical code has to be designed or an existence and uniqueness theorem needs to be proved. They treat the variational principle as a theorem to be proved in their postulation scheme. Very strange and somehow clumsy expressions are used like: theorem of the principle of virtual work which is rather an oxymoron. Their attitude (see the section on variational principles in Truesdell and Toupin [163]) is that a variational formulation cannot be generally obtained. If they exist, they are considered as mathematical curiosities that merely facilitate the work of the mathematicians. For them the search for variational principles is a secondary task relegated to the applied mathematicians.

On the contrary the supporters of variational postulations behave as if their point of view were the only one possible: they do not even care to announce that they use it as, in their opinion, everybody has to do so. To these supporters are directed the words of Piola which we already cited:

"Somebody could here object that this [i.e. the variational foundations of Analytical Mechanics] is a very old knowledge, which does not deserve to be newly promulgated by me: however [it seems that my efforts are needed] as my beautiful theories [after being published] are then criticized."

Actually the elitist attitude of many supporters of variational postulations is the true cause of the frequent rediscoveries of the same variational principles in different times and the loss of the information about their first historical appearance. Variational principles have to be regarded as the most powerful heuristic tool in applied mathematics. The wise attitude of Hamilton and Rayleigh consisted in refraining from the effort of describing dissipative phenomena directly and explicitly by means of the least action principle, but including them in the 
picture only in a second step, by means of the introduction of a suitable dissipation functional. Of course this heuristic attitude does not imply that a purely variational formulation of given model cannot be obtained, at worst by embedding the original space of configurations in a wider one. When this further step can be performed then the value of the improved mathematical model will increase.

In this context we found interesting the works Carcaterra and Sestieri [18], Carcaterra et al. [19], Culla et al. [34], Carcaterra [20], Carcaterra and Akai [22], which were initially motivated by the need to develop innovative technological solutions. In these papers it is proven that a conservative system can show, if one restricts his attention to a subset of its degrees of freedom, an apparent dissipative behavior. Actually in suitably designed conservative systems the energy may flow from some primary degrees of freedom into a precise set of other (secondary or hidden) ones, and remain there trapped for a very long (from the point of view of practical application: infinite) time. Therefore, in some cases, a non-conservative description of a primary system, including an ad-hoc dissipation functional, is a realistic and effective modeling simplification, even if the true and complete system is actually Hamiltonian and conservative. The greatest advantage in variational based models is that, if the action functional is well-behaved, they always produce intrinsically well-posed mathematical problems. Somebody claimed that this is a purely mathematical requirement: actually this is not the case. It is a "physical" prescription that a model could give a "unique" provision of the modalities of occurrence of a physical phenomenon!

There is also a practical advantage in the variational formulation of models as they are easily transformed into numerical codes. Of course after having considered Lagrangian systems (the evolution of which are governed by a least action functional) the study of nonLagrangian ones (for which such a functional may not exist) may appear very difficult. It is often stated that dissipation cannot be described by means of a least action principle. This is not exactly true, as it is possible to find some action functionals for a large class of dissipative systems (see e.g. Maugin [99],Vujanovic and Jones [166] or Moiseiwitsch [113]). However it is true that not every conceived system can be regarded as a Lagrangian one. This point is mathematically delicate and will be only evoked here (see e.g. Santilli [134] for more details). In general, a non-Lagragian system can be regarded as Lagrangian in two different ways: i) because it is an approximation of a Lagrangian system (see the case of Cattaneo equation for heat propagation in e.g. Vujanovic and Jones[166]), and this approximation leads to cancel the lacking part of the true action functional; ii) because the considered system is simply a subsystem of a larger one which is truly Lagrangian. (see e.g. Carcaterra and Sestieri [18], Carcaterra et al. [19] Carcaterra [20], Carcaterra ans Akai[22] [22]). The assumption that variational principles can be used only for non-dissipative systems is contradicted by, e.g., Bourdin et al.[11], Maugin and Trimarco [98] or Rinaldi and Lai [128] where variational principles modeling dissipative phenomena occurring in damage and fracture are formulated. In our opinion models for surface phenomena in presence of thermodynamical phenomena and diffusion or phase transitions in solids developed e.g. in McBride et al [103, 104], Steeb and Diebels [156] and Steinmann et al. [158] or for growth phenomena in living tissues such as those presented in [95] (with suitable modifications!) should be formulated in a variational form.

One should not believe that the aforementioned considerations are limited to the description of mechanical phenomena only: actually the formulation of variational principles proved to be a powerful tool in many different research fields. In the following list (which cannot be exhaustive) we simply want to indicate the enormous variety of phenomena which were considered, up to now, from the variational point of view, by citing only those few works among the many available in the literature that are more familiar to us:

- for biological evolutionary phenomena (see e.g. Edwards [56], Klimek et al. [79] and references therein);

- for the mathematical study of mutation and selection phenomena in species evolution (see e.g. Baake and Georgii [4]);

- for some phenomena of solid/solid phase transitions in plates and shells (see e..g. to Eremeyev Pietraszkiewicz et al. [126], Eremeev et al. [58], Eremeyev and Pietraszkiewicz [59] );

- for mechanical vibration control (see e.g. Carcaterra and Akai [22]);

- for electromagnetic phenomena (see e.g. Daher and Maugin [37] and references therein);

- for vibration control using distributed arrays of piezoelectric actuators (see e.g. dell'Isola Vidoli [48, 49]);

- for interfacial phenomena (see e.g. Eremeyev and Pietraszkiewicz [59], [35] Steigmann and Ogden [153], Daher and Maugin [38] and references therein);

- for the theory of membranes and rods (see e.g. Steigmann and Faulkner [155]);

- for mechanical phenomena involving different length scales (see e.g. Steigmann [154], dell'Isola et al. [51] and references therein);

- for phase transition phenomena in fluids (see Seppecher $[67,141,142,143,144]$ or Casal and Gouin [26, 27]);

- for damage and fracture phenomena (see e.g. Francfort and Marigo [66], Yang and Misra [110, 111], Contrafatto and Cuomo $[31,32,33]$, [36], Rinaldi and Lai [128] and Del Piero [55]) ; 


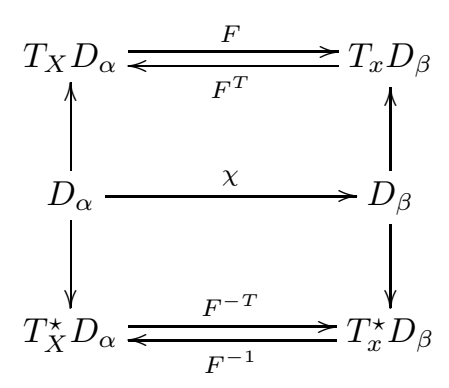

Figure 1: Diagram of Figure 1.

- for some phenomena related to fluid flow in deformable porous media (see e.g. to dell'Isola et al. [50], dell'Isola et al. [51], Sciarra et al. [136, 137, 138], Quiligotti et al. [127]);

- for some piezoelectromechanical or magnetoelastic coupling phenomena (see e.g. to Barham et al. [6], Maurini et al. [101], Maugin and Attou [97], Maurini, et al. [102], dell'Isola and Vidoli [48, 49]).

\section{Acknowledgments}

F.d.I. would like to thank his students of the course "Meccanica Analitica per Fisici" which he taught in the Academic Year 2010/2011 at the Universitï¡ di Napoli "Federico II" (his Alma Mater) and the students of the Doctoral School in Theoretical and Applied Mechanics of the Universitï¡œ di Roma "La Sapienza". Their demanding attitude towards the Professor obliged him -after having searched unsuccessfully in the literature- to write a paper where he had to prove that the Lagragian principle of least action can be the basis of the study of capillary fluids also. The ideas expressed by Pierre Seppecher during years of collaboration also greatly influenced this paper, even if it is not sure that he will approve all presented conclusions. Also the fruitful discussions with Prof. Carlo Massimo Casciola were very helpful.

This work was supported by the International Research Center M\&MoCS. V.A.E. was supported by the RFBR [grant number 12-0100038]. A.M. was supported by the project BQR 2013 "Matï¿œriaux Mïœœso et Micro-Hïœetïœœrogïœœnes: Optimisation par Modï¿œles de Second Gradient et Applications en Ingï œœnierie" [BQR 2013-0054].

\section{Appendix A. Piola transformations and the formula of material derivative}

\subsection{Geometric framework}

Let $\chi$ be a $C^{2}$-diffeomorphism between the domains $D_{\alpha}$ and $D_{\beta}$. The following notations will be adopted

$$
F:=\nabla \chi, \quad J:=\operatorname{det} F, \quad F^{-T}:=\left(F^{-1}\right)^{T}
$$

These fields are all defined in $D_{\alpha}$. Conversely, the fields

$$
F^{-1}, \quad J^{-1}:=\operatorname{det} F^{-1}, \quad F^{T}
$$

are obviously defined on $D_{\beta}$. These relations are summed up in the following diagram of Figure 1 in which $T_{p} D$ and $T_{p}^{\star} D$ denote, respectively, the tangent and cotangent plane to $D$ at $p$. For every tensor field $T_{\alpha}$ defined in $D_{\alpha}$, and for every tensor field $T_{\beta}$ defined in $D_{\beta}$, we use the notations

$$
T_{\alpha}^{\overrightarrow{(\beta)}}:=T_{\alpha} \circ \chi^{-1}, \quad T_{\beta}^{(\overrightarrow{(\alpha)}}:=T_{\beta} \circ \chi
$$

We will say that $T_{\alpha}^{(\vec{\beta})}$ is the field $T_{\alpha}$ displaced in $D_{\beta}$ and conversely. These relations are exemplified in the diagram of Figure 2 in the specific case of two vectors fields. 


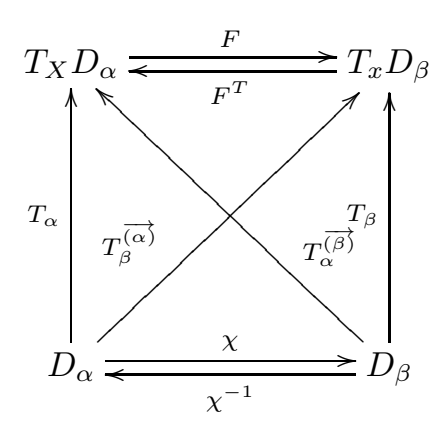

Figure 2: Diagram of Figure 2.

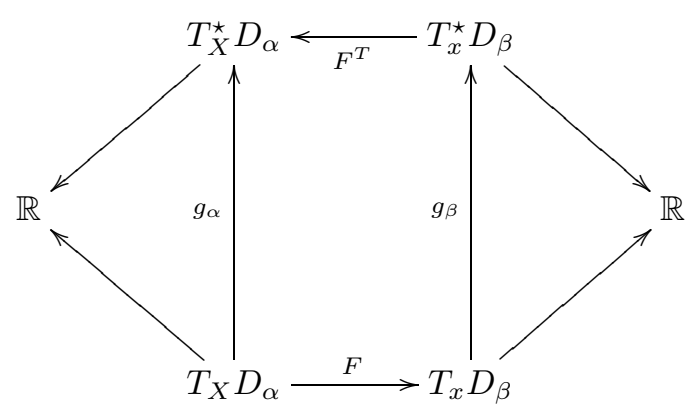

Figure 3: Diagram of Figure 3.

\subsection{Transposition of linear mappings}

The transposed $F^{T}$ of the linear mapping $F$ from the vector space $T_{X} D_{\alpha}$ to the vector space $T_{x} D_{\beta}$ is defined as the unique linear mapping from $T_{x}^{\star} D_{\beta}$ to $T_{X}^{\star} D_{\alpha}$ such that for every couple $(V, l) \in T_{X} D_{\alpha} \times T_{x}^{\star} D_{\beta}$

$$
\langle l, F V\rangle_{\left(T_{x}^{\star} D_{\beta}, T_{x} D_{\beta}\right)}=\left\langle F^{T} l, V\right\rangle_{\left(T_{X}^{\star} D_{\alpha}, T_{X} D_{\alpha}\right)}
$$

where the bracket denotes the duality product. If both $D_{\alpha}$ and $D_{\beta}$ are equipped with an inner product on their tangent space at each point $^{10}$, then tangent and cotangent space can be identified. Let us denote by $g_{\alpha}$ and $g_{\beta}$ the fields of metric defined, respectively, on $D_{\alpha}$ and $D_{\beta}$. Through $g_{\beta}$ a vector $w$ can be associated to any covector $l$, more precisely:

$$
\forall l \epsilon T_{x}^{\star} D_{\beta}, \exists w \epsilon T_{x} D_{\beta}, \quad l=g_{\beta} w
$$

Therefore the equality between the duality bracket can be rewritten

$$
\left\langle g_{\beta} w, F V\right\rangle_{\left(T_{x}^{\star} D_{\beta}, T_{x} D_{\beta}\right)}=\left\langle F^{T} g_{\beta} w, V\right\rangle_{\left(T_{X}^{\star} D_{\alpha}, T_{X} D_{\alpha}\right)}
$$

This construction can be summarized by the diagram of Figure 3 .

Once bases are introduced in $T_{X} D_{\alpha}$ and $T_{x} D_{\beta}$, we can represent vectors, tensors and inner products in terms of their components. The following relation is written in the domain $D_{\beta}$, hence quantities defined on $D_{\alpha}$ have to be transported:

$$
g_{a b} w^{b}\left(F_{A}^{a} V^{A}\right)^{\overrightarrow{(\beta)}}=V^{A}\left(F^{T}\right)_{A}^{a} g_{a b} w^{b}\left(V^{A}\right)^{\overrightarrow{(\beta)}} \quad \forall V^{A}, \forall w^{b}
$$

which implies

$$
g_{a b}\left(\left(F_{A}^{a}\right)^{\overrightarrow{(\beta)}}-\left(F^{T}\right)_{A}^{a}\right) V^{A} w^{b}=0 \quad \forall V^{A}, \forall w^{b}
$$

Therefore we have

$$
\left(F_{M}^{i}\right)^{\overrightarrow{(\beta)}}=\left(F^{T}\right)_{M}^{i},
$$

and, conversely,

$$
\left(F^{-1}\right)_{i}^{M(\alpha)}=\left(F^{-T}\right)_{i}^{M} .
$$

\footnotetext{
${ }^{10}$ In others terms, if both $D_{\alpha}$ and $D_{\beta}$ are Riemannian manifolds.
} 
Let us now consider the following inner product (with a slight abuse of notation)

$$
\langle F V, F W\rangle_{T_{x} D_{\beta}}
$$

where $F$ is the same linear mapping as before. By considering the transposed mapping one gets

$$
\langle F V, F W\rangle_{T_{x} D_{\beta}}=\left\langle F^{T} F V, W\right\rangle_{T_{X} D_{\alpha}}
$$

which in terms of components becomes

$$
\left(g_{a b}\right)^{\overrightarrow{(\alpha)}} F_{A}^{a} V^{A} F_{B}^{b} W^{B}=g_{C B}\left(F^{T} F\right)_{B}^{C} V^{A} W^{B}
$$

therefore

$$
\left(F^{T} F\right)_{M N}=\left(g_{a b}\right)^{\overrightarrow{(\alpha)}} F_{M}^{a} F_{N}^{b}=\left(F_{M a}\right)^{(\alpha)} F_{N}^{a},
$$

or more simply, dropping the change of domain:

$$
\left(F^{T} F\right)_{M N}=F_{M a} F_{N}^{a} \quad ; \quad\left(F^{T} F\right)^{M N}=F_{a}^{M} F^{a N}
$$

\subsection{Piola transformation for virtual work and stress tensors}

We call virtual displacement stemming from $\chi$ a vector field $\delta \chi$ defined in $D_{\alpha}$ and such that, for every $X$ in $D_{\alpha}$, the vector $\delta \chi(X)$ belongs to the tangent space at the point $\chi(X)$. We will denote by $\mathcal{D}$ the space of such virtual displacements:

$$
\mathcal{D}=\left\{\delta \chi: D_{\alpha} \rightarrow T D_{\beta}, X \mapsto \delta \chi(X)\right\}
$$

A virtual work functional must obviously be identified as a linear and continuous functional defined on $\mathcal{D}$ (for a detailed discussion of this point see dell'Isola et al. [54] and references therein), i.e to an element of $\mathcal{D}^{\star}$ the dual space of $\mathcal{D}$

$$
\mathcal{D}^{\star}=\{\mathcal{W}: \mathcal{D} \rightarrow \mathbb{R}, \delta \chi \mapsto W\}
$$

Because of a representation theorem due to Schwartz [135] we can state that for any virtual work functional $\mathcal{W}$ defined in $D_{\alpha}$ there exist $N$ regular fields $\underset{\gamma}{\mathrm{P}}($ where $\gamma=1, \ldots N)$ such that

$$
\mathcal{W}(\delta \chi)=\sum_{\gamma=1}^{N} \underset{D_{\alpha}}{\Delta} \underset{\gamma}{\mathrm{P}} \nabla_{\alpha}(\delta \chi) d V_{\alpha}
$$

where

$$
\underset{\gamma}{\nabla_{\alpha}}=D_{\alpha} \rightarrow \otimes^{\gamma} T^{\star} D_{\alpha} \otimes T D_{\beta} \quad ; \quad \underset{\gamma}{\mathrm{P}}=D_{\alpha} \rightarrow \otimes^{\gamma} T D_{\alpha} \otimes T^{\star} D_{\beta}
$$

Modifying slightly the nomenclature introduced by Truesdell and Toupin [163] we can call $\underset{\gamma}{\mathrm{P}}$ the $\gamma-$ th order Piola stress tensor. Now, following Piola [121], we can transport the field $\delta \chi$ on $D_{\beta}$ and define the corresponding Cauchy stress tensors $\underset{\gamma}{\mathrm{T}}$ by means of the equality

$$
\underset{D_{\alpha}}{\Delta}{ }_{\gamma} \nabla_{\gamma}(\delta \chi) d V_{\alpha}:=\underset{D_{\beta}}{\operatorname{T}} \underset{\gamma}{\nabla_{\beta}}\left(\delta \chi^{\overrightarrow{(\beta)}}\right) d V_{\beta} \quad \forall \delta \chi \in \mathcal{D}
$$

in which

$$
\underset{\gamma}{\nabla_{\beta}}=D_{\beta} \rightarrow \otimes^{\gamma} T^{\star} D_{\beta} \otimes T D_{\beta} \quad ; \quad \underset{\gamma}{\mathrm{T}}=D_{\alpha} \rightarrow \otimes^{\gamma} T D_{\beta} \otimes T^{\star} D_{\beta} .
$$

To prove that such a tensor exists, and to get its representation, let us write component-wise the previous equation:

$$
\underset{D_{\alpha}}{\Delta} \mathrm{P}_{i}^{A_{1} \ldots A_{\gamma}}(\delta \chi)_{, A_{1} \ldots A_{\gamma}}^{i} d V_{\alpha}=\underset{D_{\beta}}{\Delta \mathrm{T}_{i}^{j_{1} \ldots j_{\gamma}}}\left(\delta \chi^{(\overrightarrow{\beta)})}\right)_{, j_{1} \ldots j_{\gamma}}^{i} d V_{\beta} \quad \forall \delta \chi \in \mathcal{D} .
$$

Then using the chain rule the derivatives:

$$
\left(\delta \chi^{\overrightarrow{(\beta)}}\right)_{, j_{1} \ldots j_{\gamma}}^{i}=\left((\delta \chi)_{, A_{1} \ldots A_{\gamma}}^{i}\right)^{\overrightarrow{(\beta)}}\left(F^{-1}\right)_{j_{1}}^{A_{1}} \ldots\left(F^{-1}\right)_{j_{\gamma}}^{A_{\gamma}}
$$


and a change of variable in the second integral, we obtain

$$
\underset{D_{\alpha}}{\Delta} \mathrm{P}_{i}^{A_{1} \ldots A_{\gamma}}(\delta \chi)_{, A_{1} \ldots A_{\gamma}}^{i} d V_{\alpha}=\underset{D_{\alpha}}{\Delta} J\left(\mathrm{~T}_{i}^{j_{1} \ldots j_{\gamma}}\left(F^{-1}\right)_{j_{1}}^{A_{1}} \ldots\left(F^{-1}\right)_{j_{\gamma}}^{A_{\gamma}}\right)^{\overrightarrow{(\alpha)}}(\delta \chi)_{, A_{1} \ldots A_{\gamma}}^{i} d V_{\alpha} \quad \forall \delta \chi \in \mathcal{D}
$$

which is equivalent to the following Piola formula for transformation of stress tensors

$$
\mathrm{P}_{i}^{A_{1} \ldots A_{\gamma}}=J\left(\mathrm{~T}_{i}^{j_{1} \ldots j_{\gamma}}\left(F^{-1}\right)_{j_{1}}^{A_{1}} \ldots\left(F^{-1}\right)_{j_{\gamma}}^{A_{\gamma}}\right)^{\overrightarrow{(\alpha)}}
$$

or, using the transformation $(37)$ :

$$
\underset{\gamma}{\mathrm{P}}=J\left(\begin{array}{c}
\mathrm{T} \\
\gamma
\end{array}\right)^{\overrightarrow{(\alpha)}} \underbrace{F^{-T} \ldots F^{-T}}_{\gamma} .
$$

With simple algebra we also get

$$
J^{-1}\left(\mathrm{P}_{i}^{A_{1} \ldots A_{\gamma}} F_{A_{1}}^{i_{1}} \ldots F_{A_{\gamma}}^{i_{\gamma}}\right)^{(\overrightarrow{\beta)}}=\mathrm{T}_{\gamma i}^{i_{1} \ldots i_{\gamma}}
$$

or, using the transformation (36) :

$$
\underset{\gamma}{\mathrm{T}}=J^{-1}(\underset{\gamma}{P})^{\overrightarrow{(\beta)}} \underbrace{F^{T} \ldots F^{T}}_{\gamma} .
$$

\subsection{Piola transformation for divergence}

For any tensor field $T_{\alpha}$ the following equality holds (for a proof see e.g. dell'Isola et al. [53] or Hughes and Marsden [96]).

$$
\nabla_{\alpha} \cdot T_{\alpha}=J\left(\nabla_{\beta} \cdot\left(J^{-1} T_{\alpha}^{(\overrightarrow{\beta)}} F^{T}\right)\right)^{\overrightarrow{(\alpha)}}
$$

which obviously implies, vice versa,

$$
\left(\nabla_{\alpha} \cdot T_{\alpha}\right)^{\overrightarrow{(\beta)}}=J^{\overrightarrow{(\beta)}} \nabla_{\beta} \cdot\left(J^{-1} T_{\alpha}^{\vec{\beta})} F^{T}\right) .
$$

In components this relation reads (where $X^{L}$ and $x^{j}$ denote the components of the position vector in $D_{\alpha}$ and $D_{\beta}$ respectively)

$$
\left(\frac{\partial T_{\alpha}^{A}}{\partial X^{A}}\right)^{\overrightarrow{(\beta)}}=J^{\overrightarrow{(\beta)}} \frac{\partial}{\partial x^{a}}\left(J^{-1}\left(T_{\alpha}^{A} F_{A}^{a}\right)^{\overrightarrow{(\beta)}}\right) .
$$

Similarly we have that the following relationship, in some sense inverse of the relation (38):

$$
\nabla_{\beta} \cdot T_{\beta}=J^{-1}\left(\nabla_{\alpha} \cdot\left(J T_{\beta}^{(\alpha)} F^{-T}\right)\right)^{\overrightarrow{(\beta)}}
$$

\subsection{The Piola-Ricci-Bianchi condition}

The equation (40) was first found, without the help of tensor calculus, by Piola [121]. In the case where $T_{\beta}$ reduces to the identity, the former equation takes the following form

$$
\nabla \cdot\left(J F^{-T}\right)=0
$$

which in components can be written

$$
\frac{\partial}{\partial X^{A}}\left(J\left(F^{-1}\right)_{i}^{A}\right)=0
$$

Equation (41) is a particular case of the Bianchi condition for the Ricci curvature tensor, when interpreting Lagrangian coordinates as a chart for the Eulerian configuration of the body. Fromthe Piola-Ricci-Bianchi condition

$$
\frac{\partial}{\partial X^{A}}\left(J\left(F^{-1}\right)_{i}^{A}\right)=0
$$




$$
\begin{aligned}
J_{, A}\left(F^{-1}\right)_{i}^{A}+J\left(F^{-1}\right)_{i, A}^{A} & =0 \\
\left(F^{-1}\right)_{i, A}^{A} & =-J^{-1}\left(\frac{\rho_{0}}{\rho}\right)_{, A}\left(F^{-1}\right)_{i}^{A}=-\rho_{0} J^{-1}\left(-\frac{1}{\rho^{2}}\right) \rho_{, A}\left(F^{-1}\right)_{i}^{A}=\left(\frac{1}{\rho}\right) \rho_{, A}\left(F^{-1}\right)_{i}^{A} .
\end{aligned}
$$

In conclusion

$$
\left(F^{-1}\right)_{i, A}^{A}=\frac{\rho_{, A}}{\rho}\left(F^{-1}\right)_{i}^{A}=\frac{\rho_{, i}}{\rho}
$$

\subsection{Piola transformation for double divergence}

To obtain the Eulerian form for balance equation of the capillary fluids we need to apply the divergence twice to calculate the transformation

of double Lagrangian divergence. We proceed as follows: the equality (39) implies that (remark: we assume that the tensor $T_{\alpha}^{A B}$ is symmetric)

$$
\left(\frac{\partial T_{\alpha}^{A B}}{\partial X^{B}}\right)^{\overrightarrow{(\beta)}}=J^{\overrightarrow{(\beta)}} \frac{\partial}{\partial x^{b}}\left(J^{-1}\left(T_{\alpha}^{A B} F_{B}^{b}\right)^{\overrightarrow{(\beta)}}\right)
$$

Then

$$
\begin{aligned}
\left(\frac{\partial}{\partial X^{A}}\left(\frac{\partial T_{\alpha}^{A B}}{\partial X^{B}}\right)\right)^{\overrightarrow{(\beta)}} & =J^{\overrightarrow{(\beta)}} \frac{\partial}{\partial x^{a}}\left(J^{-1}\left(\left(\frac{\partial T_{\alpha}^{A B}}{\partial X^{B}}\right)^{\overrightarrow{(\beta)}}\left(F_{A}^{a}\right)^{\overrightarrow{(\beta)}}\right)\right) \\
& =J^{\overrightarrow{(\beta)}} \frac{\partial}{\partial x^{a}}\left(J^{-1}\left(J^{\overrightarrow{(\beta)}} \frac{\partial}{\partial x^{b}}\left(J^{-1}\left(T_{\alpha}^{A B} F_{B}^{b}\right)^{\overrightarrow{(\beta)}}\right)\left(F_{A}^{a}\right)^{\overrightarrow{(\beta)}}\right)\right) \\
& =J^{\overrightarrow{(\beta)}} \frac{\partial}{\partial x^{a}}\left(\frac{\partial}{\partial x^{b}}\left(J^{-1}\left(T_{\alpha}^{A B} F_{B}^{b}\right)^{\overrightarrow{(\beta)}}\right)\left(F_{A}^{a}\right)^{\overrightarrow{(\beta)}}\right) .
\end{aligned}
$$

In conclusion we have:

$$
\left(\frac{\partial}{\partial X^{A}}\left(\frac{\partial T_{\alpha}^{A B}}{\partial X^{B}}\right)\right)^{\overrightarrow{(\beta)}}=J^{(\overrightarrow{\beta)}} \frac{\partial}{\partial x^{a}}\left(\frac{\partial}{\partial x^{b}}\left(J^{-1}\left(T_{\alpha}^{A B} F_{B}^{b}\right)^{(\vec{\beta})}\right)\left(F_{A}^{a}\right)^{\overrightarrow{(\beta)}}\right) .
$$

\subsection{Piola transformation for normals}

For normals we have the following formula (see e.g. dell'Isola et al. [51])

$$
N_{\alpha}^{\overrightarrow{(\beta)}}=\frac{\left(J^{-1} F^{T}\right) N_{\beta}}{\left\|\left(J^{-1} F^{T}\right) N_{\beta}\right\|}
$$

while, for the passage from $\alpha$ to $\beta$ domain, the following transformation formula for areas holds:

$$
\left(\left\|\left(J^{-1} F^{T}\right) N_{\beta}\right\|^{-1}\right)^{\overrightarrow{(\alpha)}}=\left\|\left(J F^{-T}\right) N_{\alpha}\right\|=\frac{d A_{\beta}}{d A_{\alpha}} .
$$

\subsection{Material derivative}

For the formula of material derivative we start by remarking that

$$
\left(T_{\alpha}^{(\overrightarrow{\beta)}}\right)^{\overrightarrow{(\alpha)}}=T_{\alpha}
$$

Therefore

$$
\begin{aligned}
\left(\left.\frac{\partial T_{\alpha}}{\partial t}\right|_{X}\right) & =\left(\left.\frac{\partial\left(T_{\alpha}^{(\vec{\beta})}\right)^{\overrightarrow{(\alpha)}}}{\partial t}\right|_{X}=\left(\left.\frac{\partial\left(T_{\alpha}^{(\vec{\beta})} \circ \chi\right)}{\partial t}\right|_{X}=\left(\left.\frac{\partial\left(T_{\alpha}^{\vec{\beta})}(\chi(X, t), t)\right)}{\partial t}\right|_{X}\right)\right.\right. \\
& =\left(\left.\frac{\partial\left(T_{\alpha}^{(\vec{\beta})}(x, t)\right)}{\partial t}\right|_{x} \circ \chi\right)+\left.\left(\left.\nabla_{x} T_{\alpha}^{(\overrightarrow{\beta)}}(x, t)\right|_{x} \circ \chi\right) \cdot \frac{\partial \chi}{\partial t}\right|_{X} .
\end{aligned}
$$




$$
\left(\left.\frac{\partial T_{\alpha}}{\partial t}\right|_{X}\right)^{\overrightarrow{(\beta)}}=\left(\left.\frac{\partial\left(T_{\alpha}^{\overrightarrow{(\beta)}}(x, t)\right)}{\partial t}\right|_{x}+\left.\left(\left.\nabla_{x} T_{\alpha}^{\overrightarrow{(\beta)}}(x, t)\right|_{x}\right) \cdot \frac{\partial \chi}{\partial t}\right|_{X} ^{\overrightarrow{(\beta)}}\right.
$$

\section{Appendix B. Basic kinematic formulas}

In this section some useful kinematic formulas are proven (for a complete presentation of this subject see e.g. [88]). They are the basis of the procedure on which Hamilton-Piola postulation is founded. However, because of they central role, they cannot be avoided in any case: their use can be only postponed to subsequent steps, when different postulations are attempted and indeed kinematic formulas of this type are presented in any textbook of continuum mechanics. From now on, the $\alpha$ domain will coincide with the Lagrangian set of coordinates while $\beta$ domain will coincide with the Eulerian domain and the notation $(\cdot)^{\overrightarrow{\mathcal{B}})}$ and $(\cdot)^{\overrightarrow{\mathcal{E}})}$ will be consistently used. They will be omitted occasionally for the sake of readability.

\subsection{Formulas on Eulerian mass density and its gradients}

Mass density and its gradients play a pivotal role in the strain energy of fluids. Here we gather some useful formulas relating them to $C, F$ and $\nabla F$ (we will omit the needed $\left.(\cdot)^{(\mathcal{B})},(\cdot)^{(\overrightarrow{\mathcal{E}})}\right)$ for the sake of king readability).

\subsubsection{The derivative of the determinant a matrix with respect its entries}

We start by recalling the well-known formula

$$
\frac{\partial \operatorname{det}(A)}{\partial A_{M}^{i}}=\operatorname{det} A\left(A^{-T}\right)_{i}^{M}
$$

which can be recovered by using the Laplace rule for calculating the determinant

$$
\delta_{M}^{N} \operatorname{det} A=A_{M}^{a}\left(A^{*}\right)_{a}^{N}
$$

where $\left(A^{*}\right)_{i}^{N}$ is the cofactor of the element $A_{N}^{i}$. Observing that the cofactors of all elements of the $M-t h$ row are independent of the entry $A_{M}^{i}$, together with the inversion theorem for matrices, one gets

$$
\frac{\partial \operatorname{det}(A)}{\partial A_{M}^{i}}=\left(A^{*}\right)_{i}^{M}=\operatorname{det} A\left(A^{-T}\right)_{i}^{M} .
$$

\subsubsection{Partial derivatives of $\rho, J$ and $F^{-1}$ with respect to $F$}

Once one recalls that

$$
\rho_{0} \operatorname{det} F=\rho,
$$

and having defined the cofactor of $F$ as

$$
\left(F^{*}\right)_{i}^{A} F_{A}^{j}=\operatorname{det} F \delta_{i}^{j}
$$

it is easy to deduce that

$$
\begin{aligned}
\frac{\partial J}{\partial F_{M}^{i}} & =J\left(F^{-T}\right)_{M}^{i}=\frac{\rho_{0}}{\rho}\left(F^{-T}\right)_{M}^{i}, \\
\frac{\partial \rho}{\partial F_{M}^{i}} & =-\rho\left(F^{-1}\right)_{i}^{M}, \\
\frac{\partial\left(F^{-1}\right)_{j}^{N}}{\partial F_{M}^{i}} & =-\left(F^{-1}\right)_{i}^{N}\left(F^{-1}\right)_{j}^{M} .
\end{aligned}
$$

\subsubsection{Partial derivative of mass density with respect to $C$}

To prove the identity

$$
\frac{\partial \rho}{\partial C_{M N}}=-\frac{\rho}{2}\left(F^{-1}\right)^{M a}\left(F^{-1}\right)_{a}^{N}
$$


we proceed in the following way:

$$
\frac{\partial \rho}{\partial C_{M N}}=\rho_{0} \frac{\partial(\operatorname{det} C)^{-\frac{1}{2}}}{\partial C_{M N}}=\rho_{0} \frac{\partial(\operatorname{det} C)^{-\frac{1}{2}}}{\partial \operatorname{det} C} \frac{\partial \operatorname{det} C}{\partial C_{M N}}=-\frac{\rho_{0}}{2}(\operatorname{det} C)^{-\frac{3}{2}} \frac{\partial \operatorname{det} C}{\partial C_{M N}} .
$$

In conclusion we have

$$
\frac{\partial \rho}{\partial C_{M N}}=-\frac{\rho_{0}}{2}(\operatorname{det} C)^{-\frac{1}{2}}\left(C^{-1}\right)^{M N}=-\frac{\rho}{2}\left(F^{-1}\right)^{M a}\left(F^{-1}\right)_{a}^{L N} .
$$

\subsubsection{Lagrangian and Eulerian gradients of $F^{-1}$}

Starting from

$$
\left(F^{-1}\right)_{a}^{M} F_{N}^{a}=\delta_{N}^{M}
$$

after differentiation we obtain:

$$
\left(F^{-1}\right)_{a}^{M} F_{N, O}^{a}+F_{N}^{a}\left(F^{-1}\right)_{a, O}^{M}=0
$$

which produces the following chain of equalities:

$$
\begin{aligned}
F_{N}^{a}\left(F^{-1}\right)_{a, O}^{M} & =-\left(F^{-1}\right)_{a}^{M} F_{N, O}^{a} \\
\left(F^{-1}\right)_{i, O}^{M} & =-\left(F^{-1}\right)_{i}^{A}\left(F^{-1}\right)_{a}^{M} F_{A, O}^{a}
\end{aligned}
$$

The last equality can be then multiplied by $F^{-1}$ to get the Eulerian gradient

$$
\left(F^{-1}\right)_{i, j}^{M}=-\left(F^{-1}\right)_{j}^{A}\left(F^{-1}\right)_{i}^{B}\left(F^{-1}\right)_{a}^{M} F_{B, A}^{a} .
$$

It can be useful to observe that:

$$
-\left(\rho\left(F^{-1}\right)_{i}^{M}\right)_{, j}=-\rho_{, j}\left(F^{-1}\right)_{i}^{M}-\rho\left(F^{-1}\right)_{i, j}^{M}=-\rho_{, j}\left(F^{-1}\right)_{i}^{M}+\rho\left(F^{-1}\right)_{j}^{A}\left(F^{-1}\right)_{i}^{B}\left(F^{-1}\right)_{a}^{M} F_{B, A}^{a}
$$

\subsubsection{Expression of Eulerian gradient of density in terms of $F$ and its gradients}

We start from the defining relationship:

$$
\rho=\frac{\rho_{0}}{\operatorname{det}(F)}=\rho_{0} \operatorname{det}\left(F^{-1}\right) .
$$

As it is possible to assume that $\rho_{0}$ is constant, we calculate the gradient of the density as follows

$$
\rho_{, i}=\rho_{0} \operatorname{det}\left(F^{-1}\right)_{, i}=\rho_{0} \frac{\partial \operatorname{det}\left(F^{-1}\right)}{\partial\left(F^{-1}\right)_{a}^{A}} \frac{\partial\left(F^{-1}\right)_{a}^{A}}{\partial x^{i}}=\rho_{0} \operatorname{det}\left(F^{-1}\right) F_{A}^{a}\left(F^{-1}\right)_{a, i}^{A},
$$

and finally

$$
\rho_{, i}^{(\overrightarrow{\mathcal{B}})}=\rho F_{A}^{b}\left(F^{-1}\right)_{b, B}^{A}\left(F^{-1}\right)_{i}^{B} .
$$

To summarize, from all the previous expressions we obtain the following useful formulas :

$$
\begin{aligned}
\frac{\rho_{, i}}{\rho} & =-\left(F^{-1}\right)_{a}^{A}\left(F^{-1}\right)_{i}^{B} F_{A, B}^{a}=-\left(F^{-1}\right)_{a}^{A} F_{A, i}^{a} \\
\rho_{, i} & =\rho F_{A}^{a}\left(F^{-1}\right)_{i}^{B}\left(F^{-1}\right)_{a, B}^{A}=\rho F_{A}^{a}\left(F^{-1}\right)_{a, i}^{A} \\
F_{A}^{a}\left(F^{-1}\right)_{a, M}^{A} & =\frac{\rho_{, i}}{\rho} F_{M}^{i} \\
\left(F^{-1}\right)_{j, A}^{M}\left(F^{-1}\right)_{i}^{A} & =\left(F^{-1}\right)_{j}^{M} \frac{\rho_{, i}}{\rho} .
\end{aligned}
$$

\subsubsection{Calculation of partial derivative of Eulerian gradient of mass density with respect to $F$}

We need to estimate the following partial derivative:

$$
\frac{\partial \rho_{, i}}{\partial F_{M}^{j}}=\frac{\partial}{\partial F_{M}^{j}}\left(-\rho\left(F^{-1}\right)_{a}^{A}\left(F^{-1}\right)_{i}^{B}\right) F_{A, B}^{a}
$$




$$
\begin{gathered}
\left(\frac{\partial \rho}{\partial F_{M}^{i}}\left(F^{-1}\right)_{j}^{N}\left(F^{-1}\right)_{k}^{O}+\rho\left(F^{-1}\right)_{k}^{O} \frac{\partial\left(F^{-1}\right)_{j}^{N}}{\partial F_{M}^{i}}+\rho\left(F^{-1}\right)_{j}^{N} \frac{\partial\left(F^{-1}\right)_{k}^{O}}{\partial F_{M}^{i}}\right)= \\
-\rho\left(F^{-1}\right)_{i}^{M}\left(F^{-1}\right)_{j}^{N}\left(F^{-1}\right)_{k}^{O}-\rho\left(F^{-1}\right)_{i}^{N}\left(F^{-1}\right)_{j}^{M}\left(F^{-1}\right)_{k}^{O}-\rho\left(F^{-1}\right)_{j}^{N}\left(F^{-1}\right)_{i}^{O}\left(F^{-1}\right)_{k}^{M},
\end{gathered}
$$

where we used the equalities (45) and (46), we can then conclude

$$
\frac{\partial \rho,_{i}}{\partial F_{M}^{j}}=\rho\left(\left(F^{-1}\right)_{j}^{M}\left(F^{-1}\right)_{i}^{A}\left(F^{-1}\right)_{a}^{B} F_{B, A}^{a}+\left(F^{-1}\right)_{j}^{C}\left(F^{-1}\right)_{i}^{M}\left(F^{-1}\right)_{b}^{D} F_{D, C}^{b}+\left(F^{-1}\right)_{i}^{E}\left(F^{-1}\right)_{j}^{F}\left(F^{-1}\right)_{c}^{M} F_{F, E}^{c}\right)
$$

by using (51) we get

$$
\frac{\partial \rho, i}{\partial F_{M}^{j}}=-\rho_{, i}\left(F^{-1}\right)_{j}^{M}-\rho_{, j}\left(F^{-1}\right)_{i}^{M}+\rho\left(F^{-1}\right)_{i}^{A}\left(F^{-1}\right)_{l j}^{B}\left(F^{-1}\right)_{a}^{M} F_{B, A}^{a} .
$$

Finally by substituting (49) we can conclude:

$$
\frac{\partial \rho,_{i}}{\partial F_{M}^{j}}=-\rho_{, j}\left(F^{-1}\right)_{i}^{M}-\left(\rho\left(F^{-1}\right)_{j}^{M}\right)_{, i}
$$

\subsubsection{The derivatives of $(\beta)^{\overrightarrow{(\mathcal{B})}}$ with respect $F$ and $\nabla F$}

We start from a direct expression for $(\beta)^{\overrightarrow{(\mathcal{B})}}$ :

$$
(\beta)^{\overrightarrow{(\mathcal{B})}}=(\nabla \rho \cdot \nabla \rho)^{\overrightarrow{(\mathcal{B})}}=\left(g^{a b} \rho_{, a} \rho_{, b}\right)^{\overrightarrow{(\mathcal{B})}}
$$

which implies that

$$
\begin{aligned}
\frac{\partial}{\partial F}(\beta)^{\overrightarrow{(\mathcal{B})}} & =2(\nabla \rho)^{\overrightarrow{(\mathcal{B})}} \cdot \frac{\partial(\nabla \rho)^{\overrightarrow{(\mathcal{B})}}}{\partial F}, \\
\frac{\partial}{\partial \nabla F}(\beta)^{\overrightarrow{(\mathcal{B})}} & =2(\nabla \rho)^{\overrightarrow{(\mathcal{B})}} \cdot \frac{\partial(\nabla \rho)^{(\overrightarrow{\mathcal{B}})}}{\partial \nabla F} .
\end{aligned}
$$

Then using (55) and (52) we get easily:

$$
\begin{aligned}
\frac{\partial \beta}{\partial F_{M}^{i}} & =2 g^{a b} \rho_{, a} \frac{\partial(\rho, b)^{\overrightarrow{\mathcal{B}})}}{\partial F_{M}^{i}} \\
\frac{\partial(\beta)^{\overline{(\mathcal{B})}}}{\partial F_{M}^{i}} & =-2 g^{a b}\left(\rho_{, a} \rho_{, i}\left(F^{-1}\right)_{b}^{M}+\rho_{, a}\left(\rho\left(F^{-1}\right)_{i}^{M}\right)_{, b}\right)^{\overrightarrow{(\mathcal{B})}}
\end{aligned}
$$

Similarly, using (54) and (51) we obtain

$$
\frac{\partial(\beta)^{\overrightarrow{(\mathcal{B})}}}{\partial F_{M, N}^{i}}=2 g^{a b}\left(\rho_{, a}\right)^{\overrightarrow{(\mathcal{B})}} \frac{\partial\left(\rho,_{b}\right)^{\overrightarrow{(\mathcal{B})}}}{\partial F_{M, N}^{i}}=-2 g^{a b}\left(\rho \rho_{, a}\left(F^{-1}\right)_{i}^{M}\left(F^{-1}\right)_{b}^{N}\right)^{\overrightarrow{(\mathcal{B})}} .
$$

\subsection{Derivatives of $C, C^{-1}, \nabla C$ and $\nabla C^{-1}$ with respect to $F$ and $\nabla F$}

\subsubsection{Computation of $\frac{\partial C_{M N}}{\partial F_{P}^{i}}$}

$$
\begin{aligned}
\frac{\partial C_{M N}}{\partial F_{P}^{i}} & =g_{a b} \frac{\partial}{\partial F_{P}^{i}}\left(F_{M}^{a} F_{N}^{b}\right)=g_{a b}\left(\frac{\partial F_{M}^{b}}{\partial F_{P}^{i}} F_{N}^{a}+F_{M}^{b} \frac{\partial F_{N}^{a}}{\partial F_{P}^{i}}\right) \\
& =g_{a b}\left(\delta_{i}^{b} \delta_{M}^{P} F_{N}^{a}+F_{M}^{b} \delta_{i}^{a} \delta_{N}^{P}\right)=\left(\delta_{M}^{P} F_{i N}+F_{i M} \delta_{N}^{P}\right)
\end{aligned}
$$




\subsubsection{Computation of $\frac{\partial C_{M N, O}}{\partial F_{P}^{i}}$}

$$
\begin{aligned}
\frac{\partial C_{M N, O}}{\partial F_{P}^{i}} & =\frac{\partial}{\partial F_{P}^{i}}\left(\frac{\partial F_{M}^{a}}{\partial X^{O}} F_{N a}+\frac{\partial F_{N}^{b}}{\partial X^{O}} F_{L b}\right) \\
& =\frac{\partial}{\partial F_{P}^{i}}\left(\frac{\partial F_{M}^{a}}{\partial X^{O}}\right) F_{N a}+\frac{\partial F_{M}^{b}}{\partial X^{O}} \frac{\partial F_{N b}}{\partial F_{P}^{i}}+\frac{\partial}{\partial F_{P}^{i}}\left(\frac{\partial F_{N}^{c}}{\partial X^{O}}\right) F_{M c}+\frac{\partial F_{N}^{d}}{\partial X^{O}} \frac{\partial F_{M l}}{\partial F_{P}^{d}} \\
& =g_{a b} F_{M, O}^{a} \frac{\partial F_{N}^{b}}{\partial F_{P}^{i}}+g_{c d} F_{N, O}^{c} \frac{\partial F_{M}^{d}}{\partial F_{P}^{i}}=g_{a b} F_{M, O}^{a} \delta_{i}^{b} \delta_{P}^{N}+g_{c d} F_{N, O}^{c} \delta_{i}^{d} \delta_{P}^{M} \\
& =F_{i M, O} \delta_{P}^{N}+F_{i N, O} \delta_{P}^{M} .
\end{aligned}
$$

\subsubsection{Computation of $\frac{\partial C_{M N}^{-1}}{\partial F_{P}^{i}}$}

$$
\frac{\partial\left(C^{-1}\right)_{M N}}{\partial F_{P}^{i}}=\frac{\partial\left(\left(F^{-1}\right)_{a M}\left(F^{-1}\right)_{N}^{a}\right)}{\partial F_{P}^{i}}=\frac{\partial\left(\left(F^{-1}\right)_{a M}\right)}{\partial F_{P}^{i}}\left(F^{-1}\right)_{N}^{a}+\left(F^{-1}\right)_{b M} \frac{\partial\left(\left(F^{-1}\right)_{N}^{b}\right)}{\partial F_{P}^{i}} .
$$

Using equation (46) we obtain

$$
\frac{\partial\left(C^{-1}\right)_{M N}}{\partial F_{P}^{i}}=-\left(F^{-1}\right)_{M i}\left(F^{-1}\right)_{a}^{P}\left(F^{-1}\right)_{N}^{a}-\left(F^{-1}\right)_{N i}\left(F^{-1}\right)^{b P}\left(F^{-1}\right)_{b M}
$$

8.2.4 Computation of $\frac{\partial C_{M N, O}^{-1}}{\partial F_{P}^{i}}$

$$
\begin{aligned}
\frac{\partial C_{M N, O}^{-1}}{\partial F_{P}^{i}} & =\left(\left(F^{-1}\right)_{M a, O} \frac{\partial\left(F^{-1}\right)_{N}^{a}}{\partial F_{P}^{i}}+\frac{\partial\left(F^{-1}\right)_{M b}}{\partial F_{P}^{i}}\left(F^{-1}\right)_{N, O}^{b}\right) \\
& =-\left(\left(F^{-1}\right)_{N i}\left(F^{-1}\right)^{a P}\left(F^{-1}\right)_{M a, O}+\left(F^{-1}\right)_{M i}\left(F^{-1}\right)^{a P}\left(F^{-1}\right)_{a N, O}\right) \\
& =-\left(F^{-1}\right)^{a P}\left(\left(F^{-1}\right)_{N i}\left(F^{-1}\right)_{M a, O}+\left(F^{-1}\right)_{M i}\left(F^{-1}\right)_{a N, O}\right) .
\end{aligned}
$$

\subsubsection{Computation of $\frac{\partial C_{M N, O}}{\partial F_{P, Q}^{i}}$}

The computation is straightforward

$$
\frac{\partial C_{M N, O}}{\partial F_{P, Q}^{i}}=\frac{\partial}{\partial F_{P, Q}^{i}}\left(F_{M, O}^{a} F_{N a}+F_{N, O}^{b} F_{M b}\right)=\left(\delta_{i}^{a} \delta_{M}^{P} \delta_{O}^{Q} F_{N a}+\delta_{i}^{b} \delta_{N}^{P} \delta_{O}^{Q} F_{M b}\right)=\left(\delta_{M}^{P} \delta_{O}^{Q} F_{N i}+\delta_{N}^{P} \delta_{O}^{Q} F_{M i}\right)
$$

\subsubsection{Computation of $\frac{\partial C_{M N, O}^{-1}}{\partial F_{P, Q}^{i}}$}

We compute the partial derivative as the following product:

$$
\frac{\partial C_{M N, O}^{-1}}{\partial F_{P, Q}^{i}}=\frac{\partial C_{M N, O}^{-1}}{\partial\left(F^{-1}\right)_{A, B}^{a}} \frac{\partial\left(F^{-1}\right)_{A, B}^{a}}{\partial F_{P, Q}^{i}} .
$$

The first term is directly proceed:

$$
\begin{aligned}
\frac{\partial C_{M N, O}^{-1}}{\partial\left(F^{-1}\right)_{P, Q}^{i}} & =\frac{\partial}{\partial\left(F^{-1}\right)_{P, Q}^{i}}\left(g_{a b}\left(F^{-1}\right)_{N}^{a}\left(F^{-1}\right)_{M, O}^{b}+\left(F^{-1}\right)_{M c}\left(F^{-1}\right)_{N, O}^{c}\right) \\
& =g_{a b} \delta_{i}^{b} \delta_{M}^{P} \delta_{Q}^{O}\left(F^{-1}\right)_{N}^{a}+\delta_{i}^{c} \delta_{N}^{P} \delta_{Q}^{O}\left(F^{-1}\right)_{M c}\left(F^{-1}\right)_{N, L}^{c} \\
& =\delta_{Q}^{O}\left[\delta_{M}^{P}\left(F^{-1}\right)_{i N}+\delta_{N}^{P}\left(F^{-1}\right)_{M i}\right]
\end{aligned}
$$

Deriving equation (48) with respect to $F_{P, Q}^{i}$ we obtain 


$$
\frac{\partial\left(F^{-1}\right)_{i, N}^{M}}{\partial F_{P, Q}^{j}}=-\left(F^{-1}\right)_{j}^{M}\left(F^{-1}\right)_{i}^{P} \delta_{N}^{Q},
$$

Combining the results and considering that

$$
\left(F^{-1}\right)_{M, N}^{i}=g^{i a} g_{M A}\left(F^{-1}\right)_{a, N}^{A}
$$

we finally have

$$
\begin{aligned}
\frac{\partial C_{M N, O}^{-1}}{\partial F_{P, Q}^{i}} & =-\delta_{A}^{O}\left[\delta_{M}^{B}\left(F^{-1}\right)_{a N}+\delta_{N}^{B}\left(F^{-1}\right)_{M a}\right]\left(F^{-1}\right)_{B i}\left(F^{-1}\right)^{a P} \delta_{Q}^{A} \\
& =-\delta_{Q}^{O}\left[\left(F^{-1}\right)_{M i}\left(F^{-1}\right)^{a P}\left(F^{-1}\right)_{a N}+\left(F^{-1}\right)_{N i}\left(F^{-1}\right)^{b P}\left(F^{-1}\right)_{M b}\right] .
\end{aligned}
$$

\section{Appendix C. Gauss divergence theorem for embedded Riemannian manifolds}

We choose a global orthonormal basis $\left(e_{i}, i=1,2,3\right)$ for the vector field of displacements in $E^{3}$, the tridimensional Euclidean space. All tensor fields will be represented by their components with respect to this basis. In this section we consider an embedded Riemannian manifold $\mathcal{M}$ in $E^{3}$. This manifold can be therefore a regular curve or surface, but will be restricted to a surface in the present discussion. As $\mathcal{M}$ can be equipped with a Gaussian coordinate systems, it is possible to introduce in the neighborhood of any point of $\mathcal{M}$ (For more details see dell'Isola et al. [54]):

- $P$, the field of projection operator on tangent space;

- $Q$ the field of projection operator on tangent space.

These projectors verify the following obvious identities:

$$
\begin{array}{cc}
\delta_{i}^{j}=P_{i}^{j}+Q_{i}^{j}, & P_{i}^{a} P_{a}^{j}=P_{i}^{j}, \\
Q_{i}^{a} Q_{a}^{j}=Q_{i}^{j}, & P_{i}^{a} Q_{a}^{j}=0 .
\end{array}
$$

In order to simplify the forthcoming calculations, instead of using curvilinear coordinates, we rather use a global Cartesian coordinate system, completed by $P$ and $Q$ in the neighborhood of $\mathcal{M}$. This technical choice is exactly the same one which allowed Germain to generalize, for second gradient materials, the results found by Green, Rivlin, Toupin and Mindlin.

The unit external normal to $\mathcal{M}$ on its border, which is denoted $\nu$, belongs to the tangent space to $\mathcal{M}$.

Using these notations the divergence theorem reads (see e.g. Spivak [159]): for any vector field $W$ defined in the vicinity of $M$

$$
\Delta_{M}\left(P_{b}^{a} W^{b}\right)_{, c} P_{a}^{c} d S=\Delta_{\partial M} W^{a} P_{a}^{b} \nu_{b} d L
$$

This theorem together with relation

$$
Q_{j, b}^{a} P_{a}^{b}=-Q_{j}^{a} P_{a, b}^{b}
$$

implies that, for any vector field $W$ defined in a neighborhood of $\mathcal{M}$,

$$
\begin{aligned}
\Delta_{M}\left(W^{a}\right){ }_{,} P_{a}^{b} d S & =\Delta_{M}\left[\left(P_{b}^{a} W^{b}\right)_{, c} P_{a}^{c}+\left(Q_{e}^{d} W^{e}\right)_{, f} P_{d}^{f}\right] d S \\
& =\Delta_{M} W^{a} Q_{a, c}^{b} P_{b}^{c} d S+\Delta_{\partial M} W^{d} P_{d}^{e} \nu_{e} d L=-\Delta_{M} W^{a} Q_{a}^{b} P_{b, c}^{c} d S+\Delta_{\partial M} W^{d} P_{d}^{f} \nu_{f} d L .
\end{aligned}
$$

\section{References}

[1] Alibert, J.J., Seppecher, P. and dell'Isola, F., Truss modular beams with deformation energy depending on higher displacement gradients. Mathematics and Mechanics of Solids, 8, 51-73 (2003).

[2] Atai, A.A. and Steigmann, D.J., On the nonlinear mechanics of discrete networks. Archive of Applied mechanics, 67, 303-319 (1997) 
[3] Auriault, J.-L., Geindreau, C. and Boutin, C., Filtration law in porous media with poor separation of scales. Transport in Porous Media, 60, 89-108 (2005) .

[4] Baake, E. and Georgii, H.-O., Mutation, selection, and ancestry in branching models: a variational approach. Journal of Mathematical Biology, 54, 257-303 (2007).

[5] Ball, J. M., Convexity conditions and existence theorems in nonlinear elasticity, Archive for Rational Mechanics and Analysis, 63 (4), 337-403 (1976).

[6] Barham, M., Steigmann, D.J., McElfresh, M. and Rudd, R.E. Limit-point instability of a magnetoelastic membrane in a stationary magnetic field. Smart Materials and Structures,17 (2008).

[7] Bassanini P., Casciola C.M., Lancia M.R., Piva R., On the trailing edge singularity and Kutta condition for 3D airfoils - European journal of mechanics. B, Fluids, 15, 6, pp. 809-830 (1996)

[8] Bedford, A., Hamilton's principle in continuum mechanics. Volume 139 di Research notes in mathematics Pitman Advanced Publishing Program, 1985.

[9] Berdichevsky, V., Variational principles of continuum mechanics. Voll.I,II, Springer, 2009.

[10] Bleustein, J.L., A note on the boundary conditions of Toupin's strain-gradient theory. International Journal of Solids and Structures, 3, 1053-1057 (1967).

[11] Bourdin, B., Francfort, G.A. and Marigo, J.-J., The variational approach to fracture. Journal of Elasticity, 91, 1-148 (2008). (The paper also appeared as a Springer book: ISBN: 978-1-4020-6394-7).

[12] Boutin, C. and Auriault, J.-L., Acoustics of a bubbly fluid at large bubble concentration. European Journal of mechanics B/fluids, 12, 367-399 (1993).

[13] Boutin, C., Hans, S. and Chesnais, C., Generalized beams and continua. Dynamics of reticulated structures. In Mechanics of Generalized Continua (131-141). Springer New York (2011).

[14] Boutin, C. and Hans, S., Homogenisation of periodic discrete medium: Application to dynamics of framed structures. Computers and Geotechnics, 30, 303-320 (2003).

[15] Cahn J.W., and Hilliard, J.E., Free Energy of a Nonuniform System. I. Interfacial Free Energy. The Journal of Chemical Physics, 28, 258-267 (1958).

[16] Cahn, J.W. and Hilliard, J.E., Free energy of a non uniform system III. The Journal of Chemical Physics, 31, 688-699 (1959).

[17] Capecchi, D. and Ruta, G.C., Piola's contribution to continuum mechanics, Archive for History of Exact Sciences, 61, 303-342 (2007).

[18] Carcaterra, A. and Sestieri A., Energy Density Equations and Power Flow in Structures. Journal of Sound and Vibration, 188, 269-282 (1995).

[19] Carcaterra, A., E. Ciappi, A. and Iafrati, E.F., Campana, Shock spectral analysis of elastic systems impacting on the water surface. Journal of Sound and Vibration, 229, 579-605(2000).

[20] Carcaterra, A., Ensemble energy average and energy flow relationships for nonstationary vibrating systems. Journal of Sound and Vibration, 288, 751-790(2005).

[21] Carcaterra, A., Akay A. and Ko, I.M., Near-irreversibility in a conservative linear structure with singularity points in its modal density. Journal of the Acoustical Society of America, 119, 2141-2149 (2006) .

[22] Carcaterra, A. and Akay, A., Theoretical foundations of apparent-damping phenomena and nearly irreversible energy exchange in linear conservative systems. Journal of the Acoustical Society of America, 12 1971-1982 (2007).

[23] Carcaterra, A. and Akay, A., Dissipation in a finite-size bath. Physical Review E, 84, 011121 (2011).

[24] Casal, P., La capillarité interne. Cahier du groupe Francais de rhéologie, 3, 31-37 (1961).

[25] Casal, P., La théorie du second gradient et la capillarité. Comptes rendus de l'Académie des Sciences Série A, 274, 1571-1574 (1972). 
[26] Casal, P. and Gouin H., Connection between the energy equation and the motion equation in Korteweg's theory of capillarity. Comptes rendus de l'Académie des Sciences Série II, 300, 231-234 (1985).

[27] Casal, P. and Gouin H., Equations of motion of thermocapillary fluids, Comptes rendus de l'Académie des Sciences Série II, 306, 99-104 (1988).

[28] Casciola C.M., Gualtieri P., Jacob B., Piva R. Scaling properties in the production range of shear dominated flows Physical review letters 95, 024503 (2005)

[29] Chesnais, C., Boutin, C and Hans, S., Wave propagation and non-local effects in periodic frame materials: Generalized continuum mechanics (In preparation).

[30] Chesnais, C., Boutin, C., Hans, S., Effects of the local resonance on the wave propagation in periodic frame structures: Generalized Newtonian mechanics. Journal of the Acoustical Society of America, 132, 2873-2886 (2012).

[31] Contrafatto, L. and Cuomo, M., A new thermodynamically consistent continuum model for hardening plasticity coupled with damage. International Journal of Solids and Structures, 39, 6241-6271 (2002).

[32] Contrafatto, L. and Cuomo, M., A framework of elastic-plastic damaging model for concrete under multiaxial stress states, International Journal of Plasticity, 22, 2272-2300 (2006).

[33] Contrafatto, L. and Cuomo, M., A globally convergent numerical algorithm for damaging elasto-plasticity based on the Multiplier method. International Journal for Numerical Methods in Engineering, 63,1089-1125 (2005).

[34] Culla, A., Sestieri, A. and Carcaterra, A., Energy flow uncertainties in vibrating systems: Definition of a statistical confidence factor. Mechanical Systems and Signal Processing, 17, 635-663(2003) .

[35] Cuomo, M. and Ventura, G., Complementary Energy Approach to Contact Problems Based on Consistent Augmented Lagrangian regularization. Mathematical and Computer Modelling, 28, 185-204 (1998)

[36] Cuomo, M. and Contrafatto, L., Stress rate formulation for elastoplastic models with internal variables based on augmented Lagrangian regularisation. International Journal of Solids and Structures, 37 3935-3964 (2000).

[37] Daher, N. and Maugin, G.A., Virtual power and thermodynamics for electromagnetic continua with interfaces. Journal of Mathematical Physics, 27, 3022-3035 (1986).

[38] Daher, N., Maugin, G.A., The method of virtual power in continuum mechanics. Application to media presenting singular surfaces and interfaces. Acta Mechanica, 60, 217-240 (1986) .

[39] de Gennes, P.G., Some effects of long range forces on interfacial phenomena. Journal de Physique Lettres, 42, L-377, L-379 (1981).

[40] dell'Isola, F. and Romano, A., On a general balance law for continua with an interface. Ricerche di Matematica, 35, 325-337 (1986).

[41] dell'Isola, F. and Romano, A., On the derivation of thermomechanical balance equations for continuous systems with a nonmaterial interface. International Journal of Engineering Science, 25, 1459-1468 (1987).

[42] dell'Isola, F. and Romano, A., A phenomenological approach to phase transition in classical field theory. International Journal of Engineering Science, 25, 1469-1475 (1987).

[43] dell'Isola, F. and Kosinski, W., Deduction of thermodynamic balance laws for bidimensional nonmaterial directed continua modelling interphase layers. Archives of Mechanics, 45, 333-359 (1993).

[44] F.dell'Isola, Gouin, H. and Seppecher, P., Radius and surface tension of microscopic bubbles by second gradient theory, Comptes rendus de l'Académie des Sciences Série IIb, 320, 211-216, (1995).

[45] dell'Isola, F. and Seppecher, P., The relationship between edge contact forces, double force and interstitial working allowed by the principle of virtual power. Comptes rendus de l'Académie des Sciences Serie IIb, 321, 303-308 (1995).

[46] dell'Isola, F. and Seppecher, P., Edge Contact Forces and Quasi-Balanced Power. Meccanica, 32, 33-52 (1997).

[47] dell'Isola, F. and Hutter, K., What are the dominant thermomechanical processes in the basal sediment layer of large ice sheets? Proceedings of the Royal Society of London. Series A: Mathematical, Physical and Engineering Sciences, 454, 1169-1195 (1972). 
[48] dell'Isola, F. and Vidoli, S. Damping of bending waves in truss beams by electrical transmission lines with PZT actuators. Archive of Applied Mechanics, 68, 626-636 (1998).

[49] dell'Isola, F. and Vidoli, S. Continuum modelling of piezoelectromechanical truss beams: an application to vibration damping. Archive of Applied Mechanics, 68, 1-19 (1998).

[50] dell'Isola, F., Guarascio, M. and Hutter, K.A., Variational approach for the deformation of a saturated porous solid. A second-gradient theory extending Terzaghi's effective stress principle. Archive of Applied Mechanics, 70, 323-337 (2000).

[51] dell'Isola, F., Madeo, A. and Seppecher, P., Boundary Conditions in Porous Media: A Variational Approach. International Journal of Solids and Structures, 46, 3150-3164 (2009).

[52] dell'Isola, F., Sciarra, G. and Vidoli, S., Generalized Hooke's law for isotropic second gradient materials. Proceedings of the Royal Society of London. Series A: Mathematical, Physical and Engineering Sciences, 465, 2177-2196 (2009).

[53] dell'Isola, F. and Placidi, L., Variational principles are a powerful tool also for formulating field theories. Variational Models and Methods in Solid and Fluid mechanics CISM Courses and Lectures, 535, 1-15 (2011).

[54] dell'Isola, F., Seppecher, P. and Madeo, A., How contact interactions may depend on the shape of Cauchy cuts in N-th gradient continua: approach á la D'Alembert. Zeitschrift für Angewandte Mathematik und Physik (ZAMP), 63, 1119-1141 (2012).

[55] Del Piero, G., A Variational Approach to Fracture and Other Inelastic Phenomena, Journal of Elasticity, 112(1), 3-77, (2013).

[56] Edwards, A.W.F., Maximisation principles in Evolutionary Biology. Philosophy of Biology, Mohan Matthen and Christopher Stephens Editors Elsevier 335-349 (2007).

[57] Evans R., The nature of the liquid-vapor interface and other topics in the statistical mechanics of non-uniform, classical fluids. Advances in Physics, 28, 143-200 (1979).

[58] Eremeev V.A., Freidin A.B. and Sharipova L.L., Nonuniqueness and stability in problems of equilibrium of elastic two-phase bodies. Doklady Physics, 48, 359-363 (2003).

[59] Eremeyev V.A. and Pietraszkiewicz W., The nonlinear theory of elastic shells with phase transitions. Journal of Elasticity, 74, 67-86 (2004).

[60] Eremeyev, V. A. and Pietraszkiewicz, W., Thermomechanics of shells undergoing phase transition. Journal of the Mechanics and Physics of Solids, 59, 1395-1412 (2011).

[61] Eremeyev V.A. and Lebedev L.P., Existence of weak solutions in elasticity. Mathematics and Mechanics of Solids, 18, 204-217 (2013).

[62] Esposito, R. and Pulvirenti, M., From particles to fluids. Handbook of mathematical fluid dynamics. Vol. III, 1-82, North-Holland, Amsterdam, 2004.

[63] Fermi, E., Pasta, J. and Ulam, S., Studies of Nonlinear Problems. Document LA-1940, 1955.

[64] Forest, S., Cordero, N.M. and Busso, E.P., First vs. second gradient of strain theory for capillarity effects in an elastic fluid at small length scales. Computational Materials Science, 50, 1299-1304 (2011).

[65] Forest, S., Micromorphic approach for gradient elasticity, viscoplasticity, and damage. Journal of Engineering Mechanics, 135, 117-131 (2009).

[66] Francfort, G.A. and Marigo, J.-J., Revisiting brittle fracture as an energy minimization problem. Journal of the Mechanics and Physics of Solids, 46, 1319-1342 (1998).

[67] Gatignol, R. and Seppecher, P., Modelisation of fluid-fluid interfaces with material properties. Journal de Mécanique Théorique et Appliquée, 225-247 (1986).

[68] Gavrilyuk, S. and Gouin, H., A new form of governing equations of fluids arising from Hamilton's principle. International Journal of Engineering Science, 37, 1495-1520 (1999).

[69] Germain, P., La méthode des puissances virtuelles en mécanique des milieux continus. Premiére partie. Théorie du second gradient, Journal de Mécanique, 12, 235-274 (1973). 
[70] Germain, P., The method of virtual power in continuum mechanics. Part 2: Microstructure. SIAM, Journal of Applied Mathematics 25, 556-575 (1973).

[71] Germain, P., Toward an analytical mechanics of materials, in:Nonlinear thermodynamical processes in continua (Eds.W.Muschik and G.A.Maugin), TUB-Dokumentation und Tagungen, Heft 61, Berlin , 198-212 (1992).

[72] Green, A.E. and Rivlin, R.S., Multipolar continuum mechanics, Archive for Rational Mechanics and Analysis, 17, 113-147 (1964).

[73] Green, A.E. aand Rivlin, R.S., Simple force and stress multipoles, Archive for Rational Mechanics and Analysis,16, 325-353 (1964).

[74] Green, A.E. and Rivlin, R.S., On Cauchy's equations of motion, Zeitschrift für Angewandte Mathematik und Physik (ZAMP),15, 290-292, (1964).

[75] Green, A.E.and Rivlin, R.S., Multipolar continuum mechanics: functional theory. I, Proceedings of the Royal Society of London. Series A: Mathematical, Physical and Engineering Sciences, 284, 303-324 (1965).

[76] Haseganu, E.M. and Steigmann, D.J., Equilibrium analysis of finitely deformed elastic networks. Computational mechanics, 17, 359-373 (1996) .

[77] Hellinger, E., Die allgemeinen Ansitze der Mechanik der Kontinua. Enz. math. Wiss. 4 , 602-694 (1972).

[78] Jacob B., Casciola C.M., Talamelli A., Alfredsson P.H., Scaling of mixed structure functions in turbulent boundary layers Physics of fluids 20 (4), 045101-045101-7 (2008)

[79] Klimek, P., Thurner, S. and Hanel, R., Evolutionary dynamics from a variational principle, Physical Review E, 82, 011901 (2010).

[80] Korteweg, D. J. and de Vries, G., On the Change of Form of Long Waves Advancing in a Rectangular Canal, and on a New Type of Long Stationary Waves. Philosophical Magazine, 39, 422-443 (1895).

[81] Kravchuk, A. and Neittaanmaki, P., Variational and quasi-variational Inequalities in mechanics. Springer (2007).

[82] Korteweg, D. J., Sur la forme que prennent les équations des mouvements des fluides si l'on tient compte des forces capillaires par des variations de densité. Arch. Néer. Sci. Exactes Sér. II, 6, 1-24 (1901).

[83] Kroner, E., Mechanics of Generalized Continua, Springer (1968).

[84] Kupershmidt B., The variational principles of Dynamics, World Scientific (1992).

[85] Landau, L.D. and Lifshitz, E.M., Quantum mechanics: Non-Relativistic Theory. Vol. 3 (3rd ed.), Pergamon Press (1977).

[86] Lanczos, C., The Variational principles of mechanics. Toronto: University of Toronto (1970).

[87] Lagrange, J.L., Mécanique Analytique, Editions Jaques Gabay, Sceaux (1788).

[88] Lebedev, L.P., Cloud, M.J., and Eremeyev, V. A., Tensor Analysis with Applications in Mechanics. New Jersey: World Scientific (2010).

[89] Leipholz, H.H.E., Six Lectures on Variational Principkes in Structural Engineering, University of Waterloo, Canada (1983).

[90] Lemons, D.S., Perfect Form: Variational principles, Methods and Applications in Elementary Physics. Princeton University Press (1997).

[91] Lippmann, H., Extremum and Variational principles in mechanics. CISM Springer Verlag (1972).

[92] Luongo, A. and Di Egidio, A., Bifurcation equations through multiple-scales analysis for a continuous model of a planar beam. Nonlinear Dynamics, 41, 171-190 (2005).

[93] Luongo, A. and Romeo, F., A Transfer-matrix-perturbation approach to the dynamics of chains of nonlinear sliding beams. Journal of Vibration and Acoustics, 128, 190-196 (2006).

[94] Luongo, A., Zulli, D. and Piccardo, G., On the effect of twist angle on nonlinear galloping of suspended cables. Computers $\mathcal{E}^{3}$ Structures, 87, 1003-1014 (2009).

[95] Madeo, A., Lekszycki, T. and dell'Isola, F., A continuum model for the bio-mechanical interactions between living tissue and bioresorbable graft after bone reconstructive surgery. Comptes rendus Mecanique, 339, 625-682 (2011). 
[96] Marsden, J. E., \& Hughes, T. J. (1983). Mathematical foundations of elasticity. Dover Publications.

[97] Maugin, G.A. and Attou, D., An asymptotic theory of thin piezoelectric plates. The Quarterly Journal of Mechanics and Applied Mathematics, 43, 347-362 (1989).

[98] Maugin, G.A. and Trimarco, C., Pseudomomentum and material forces in nonlinear elasticity: variational formulations and application to brittle fracture. Acta Mechanica 94, 1-28 (1992).

[99] Maugin, G.A., Towards an analytical mechanics of dissipative materials. Rend. Sem. Mat. Univ. Pol. Torino Etude des conditions aux limites en théorie du second gradiVol. 58, 2 (2000).

[100] Maugin, G.A., The principle of virtual power: from eliminating metaphysical forces to providing an efficient modelling tool. Continuum Mechanics and Thermodynamics, 25, 127-146 (2011).

[101] Maurini, C., dell'Isola, F and del Vescovo, D., Comparison of piezoelectronic networks acting as distributed vibration absorbers. Mechanical Systems and Signal Processing, 18, 1243-1271 (2004).

[102] Maurini, C., and Pouget, J. and dell'Isola, F., On a model of layered piezoelectric beams including transverse stress effect. International Journal of Solids and Structures, 4, 4473-4502 (2004).

[103] McBride, A.T., Javili, A., Steinmann, P. and Bargmann, S., Geometrically nonlinear continuum thermomechanics with surface energies coupled to diffusion, Journal of the Mechanics and Physics of Solids, 59, 2116-2133 (2011).

[104] McBride, A.T., Mergheim, J., Javili, A., Steinmann, P. and Bargmann, S., Micro-to-macro transitions for heterogeneous material layers accounting for in-plane stretch, Journal of the Mechanics and Physics of Solids, 60, 1221-1239 (2012).

[105] Maxwell, J.C., A treatise on electricity and magnetism Voll.I,II Oxford at the Clarendon Press (1873).

[106] Mindlin, R.D., Micro-structure in linear elasticity. Archive for Rational Mechanics and Analysis, 16, 51-78 (1964).

[107] Mindlin, R.D., Second gradient of strain and surface tension in linear elasticity. International Journal of Solids and Structures, 1, 417-438 (1965).

[108] Mindlin, R.D. and Eshel, N.N. On first strain-gradient theories in linear elasticity. International Journal of Solids and Structures, 4, 109-124 (1968).

[109] Misra, A. and Chang, C.S. , Effective Elastic Moduli of Heterogeneous Granular Solids. International Journal of Solids and Structures, $30,2547-2566(1993)$.

[110] Misra, A. and Yang, Y.,. Micromechanical model for cohesive materials based upon pseudo-granular structure. International Journal of Solids and Structures, 47, 2970-2981 (2010) .

[111] Misra, A. and Singh, V., Micromechanical model for viscoelastic-materials undergoing damage. Continuum Mechanics and Thermodynamics, 25, 1-16 (2013).

[112] Misra, A. and Ching, W.Y., Theoretical nonlinear response of complex single crystal under multi-axial tensile loading, Scientific Reports, 3 (2013).

[113] Moiseiwitsch, B.L., Variational principles. Dover (2004).

[114] Nadler, B. and Steigmann, D.J., A model for frictional slip in woven fabrics. Comptes Rendus Mecanique, 331, 797-804 (2003).

[115] Nadler, B., Papadopoulos, P. and Steigmann, D.J., Multiscale constitutive modeling and numerical simulation of fabric material, International Journal of Solids and Structures, 43, 206-221 (2006).

[116] Noll, W. Foundations of mechanics and Thermodynamics, Selected Papers. Springer-Verlag, New York (1974).

[117] Noll, W. and Truesdell, C. The Non-Linear Field Theories of mechanics, Encyclopie of Phisics, vol. III/3, Springer-Verlag, New York (1965).

[118] Piola, G., Sull'applicazione de' principj della meccanica analitica del Lagrange ai principali problemi. Memoria di Gabrio Piola presentata al concorso del premio e coronata dall'I.R. Istituto di Scienze, ecc. nella solennita del giorno 4 ottobre 1824, Milano, Imp.

Regia stamperia, 1825 
[119] Piola, G., La meccanica de' corpi naturalmente estesi: trattata col calcolo delle variazioni, Milano, Giusti, (1833).

[120] Piola, G., Nuova analisi per tutte le questioni della meccanica molecolare - del Signor Dottore Don Gabrio Piola - Ricevuta adí 21 Marzo 1835, Memorie di Matematica e di Fisica della Società Italiana delle Scienze residente in Modena, 21, pp. 155-321, (1836).

[121] Piola, G., Intorno alle equazioni fondamentali del movimento di corpi qualsivogliono, considerati secondo la naturale loro forma e costituzione - Memoria del Signor Dottor Gabrio Piola - Ricevuta adí 6 Ottobre 1845, Memorie di Matematica e di Fisica della Società Italiana delle Scienze residente in Modena, 24, pp. 1-186, (1848). Translated in this volume.

[122] Piola, G., Di un principio controverso della Meccanica analitica di Lagrange e delle molteplici sue applicazioni - Memoria postuma di Gabrio Piola - (pubblicata per cura del prof. Francesco Brioschi), Memorie dell'I.R. Istituto Lombardo di Scienze, Lettere ed Arti, 6, pp. 389-496, (1856). Translated in this volume.

[123] Poisson, S.-D., Mémoire sur l'équilibre et le mouvement des Corps solides élastiques. Mémoires de l'Institut de France T. VIII. pag. 326,400 ;

[124] Poisson, S.-D., Mémoire sur les Equations générales de l'équilibre et du mouvement des Corps solides, élastiques et fluides. Journal de l'Ecole Polytechnique, 13, 1-174 (1829).

[125] Poisson, S.-D., Nouvelle Théorie de l'Action Capillaire. Bachelier, Paris (1831)

[126] Pietraszkiewicz, W., Eremeyev, V.A. and Konopinska, V., Extended non-linear relations of elastic shells undergoing phase transitions. Zeitschrift für Angewandte Mathematik und Mechanik (ZAMM), 87, 150-159 (2007).

[127] Quiligotti, S., Maugin, G.A. and dell'Isola, F., An Eshelbian approach to the nonlinear mechanics of constrained solid-fluid mixtures, Acta Mechanica, 160, 45-60 (2003).

[128] Rinaldi, A. and Lai, Y.-C., Statistical damage theory of 2D lattices: Energetics and physical foundations of damage parameter. International Journal of Plasticity, 23, 1796-1825(2007).

[129] Rinaldi, A., Krajcinovic, D., Peralta, P. and Lai, Y.-C., Lattice models of polycrystalline microstructures: A quantitative approach. Mechanics of Materials, 40, 17-36 (2008).

[130] Rivlin, R.S. Forty years of nonlinear continuum mechanics Proc.IX Intl. Congress on Rheology Mexico (1984) reprinted In Barenblatt G.I. and Joseph D.D. Eds. Collected Papers of R.S. Rivlin Volume II Springer (1996)

[131] Rivlin, R.S. Red herrings and sundry unidentified fish in nonlinear continuum mechanics In Barenblatt G.I. and Joseph D.D. Eds. Collected Papers of R.S. Rivlin Volume II Springer (1996)

[132] Rorres, C., Completing Book II of Archimedes's On Floating Bodies. The mathematical intelligencer, 26, 32-42 (2004).

[133] Russo, L., The Forgotten Revolution. Springer Verlag (2003).

[134] Santilli, R., Foundations of theoretical mechanics II. Birkhoffian generalization of Hamiltonian mechanics. Springer (1982).

[135] Schwartz, L., Théorie des Distributions, Hermann Paris, (1973).

[136] Sciarra G., dell'Isola, F. and Hutter, K., A solid-fluid mixture model allowing for solid dilatation under external pressure. Continuum Mechanics and Thermodynamics, 13, 287-306 (2001).

[137] Sciarra, G., dell'Isola, F. and Coussy, O., Second gradient poromechanics. International Journal of Solids and Structures, 44 ,66076629 (2007).

[138] Sciarra, G., dell'Isola, F., Ianiro, N. and Madeo A., A variational deduction of second gradient poroelasticity part I: General theory. Journal of Mechanics of Materials and Structures, 3, 507-526 (2008).

[139] Sedov, L.I., Models of continuous media with internal degrees of freedom, Journal of Applied Mathematics and Mechanics, 32, 803-819 (1972)

[140] Sedov, L.I., Variational Methods of constructing Models of Continuous Media. Irreversible Aspects of Continuum Mechanics and Transfer of Physical Characteristics in Moving Fluids. Springer Vienna, 346-358 (1968). 
[141] Seppecher, P., Etude d'une Modelisation des Zones Capillaires Fluides: Interfaces et Lignes de Contact, Thése de l'Universitá Paris VI, Avril (1987).

[142] Seppecher, P., Thermodynamique des zones capillaires, Annales de Physique, 13, 13-22 (1988).

[143] Seppecher, P., Etude des conditions aux limites en théorie du second gradient : cas de la capillarité, Comptes rendus de l'Académie des Sciences, 309, 497-502 (1989).

[144] Seppecher, P., Equilibrium of a Cahn and Hilliard fluid on a wall: Influence of the wetting properties of the fluid upon the stability of a thin liquid film, European Journal of mechanics B/fluids, 12, 69-84 (1993).

[145] Seppecher, P., A numerical study of a moving contact line in Cahn-Hilliard theory, International Journal of Engineering Science, 34, 977-992 (1996).

[146] Seppecher, P., Les Fluides de Cahn-Hilliard. Mémoire d'Habilitation á Diriger des Recherches, Universitá du Sud Toulon Var (1996).

[147] Seppecher, P., Second-gradient theory : application to Cahn-Hilliard fluids, in Continuum Thermomechanics, Springer Netherlands, 379-388 (2002).

[148] Seppecher, P., Line Tension Effect upon Static Wetting, Oil and Gas Science and Technology- Rev. IFP, vol 56, 77-81 (2001).

[149] Davison, E., Soper Classical Field Theory. Dover Publications (2008).

[150] Soubestre, J. and Boutin, C., Non-local dynamic behavior of linear fiber reinforced materials, Mechanics of Materials, 55, 16-32 (2012).

[151] Sunyk, R. and Steinmann, P., On Higher Gradients in continuum-Atomistic Modelling. International Journal of Solids and Structures, 40, 6877-6896 (2003).

[152] Steigmann, D.J., Equilibrium of prestressed networks, IMA Journal of Applied Mathematics (Institute of Mathematics and Its Applications), 48, 195-215 (1992).

[153] Steigmann, D.J. and Ogden, R.W., Elastic surface-substrate interactions (1999). Proceedings of the Royal Society A: Mathematical, Physical and Engineering Sciences,455, 437-474 (1982).

[154] Steigmann, D.J. The variational structure of a nonlinear theory for spatial lattices, Meccanica, 31, 441-455(1996).

[155] Steigmann, D.J. and Faulkner, M.G. Variational theory for spatial rods. Journal of Elasticity, 33, 1-26(1993).

[156] Steeb H. and Diebels S., Modeling thin films applying an extended continuum theory based on a scalar-valued order parameter Part I: Isothermal case. International Journal of Solids and Structures, 41 5071-5085(2004).

[157] Steinmann, P., Elizondo, A. and Sunyk, R., Studies of validity of the Cauchy-Born rule by direct comparison of continuum and atomistic modelling. Modelling and Simulation in Materials Science and Engineering, 15 (2007).

[158] Steinmann, P., McBride, A.T., Bargmann, S. and Javili, A., A deformational and configurational framework for geometrically nonlinear continuum thermomechanics coupled to diffusion. International Journal of Non-Linear mechanics, 47, 215-227 (2012) .

[159] Spivak, M., A comprehensive introduction to differential geometry, Vol. I and II. Second edition. Publish or Perish, Inc., Wilmington, Del. (1979).

[160] Toupin R.A., Elastic Materials with couple-stresses. Archive for Rational Mechanics and Analysis, 11, 385-414 (1962)

[161] Toupin R.A., Theories of elasticity with couple-stress. Archive for Rational Mechanics and Analysis, 17 85-112 (1964).

[162] Truesdell, C., Essays in the Hystory of mechanics Springer Verlag (1968).

[163] Truesdell, C.and Toupin R.A., The Classical field Theories Handbuch der Physic Band III/1 Springer (1960).

[164] Van Kampen, N.G., Condensation of a classical gas with long range attraction, Physical Review, 135, A362-A369 (1964)

[165] Vailati, G., Il principio dei lavori virtuali da Aristotele a Erone d'Alessandria, Scritti (Bologna, Forni, 1897), vol. II, pp. 113-128. Atti della R. Accademia delle Scienze di Torino, vol. XXXII, adunanza del 13 giugno 1897, quaderno IG (091) 75 I - III. 1897 
[166] Vujanovic, B.D. and Jones S.E., Variational Methods in Nonconservative Phenomena. Academic Press (1989).

[167] Yang, Y., and Misra, A., Higher-order stress-strain theory for damage modeling implemented in an element-free Galerkin formulation. Computer Modeling in Engineering and Sciences, 64, 1-36 (2010).

[168] Yang, Y., and Misra, A., Micromechanics based second gradient continuum theory for shear band modeling in cohesive granular materials following damage elasticity. International Journal of Solids and Structures, 49, 2500-2514 (2012) .

[169] Yang, Y., Ching, W.Y. and Misra A., Higher-order continuum theory applied to fracture simulation of nano-scale intergranular glassy film. Journal of Nanomechanics and Micromechanics, 1, 60-71 (2011) .

[170] Yeremeyev, V.A., Freidin, A.B. and Sharipova, L.L. The stability of the equilibrium of two-phase elastic solids. Journal of Applied Mathematics and mechanics (PMM), 71, 61-84 (2007). 